\title{
Effect of Chemical Reaction on Statistical Theory of Dusty Fluid MHD Turbulent Flow for Certain Variables at Three- Point Distribution Functions
}

\author{
M. Abul Kalam Azad, M. Abu Bkar Pk, Abdul Malek \\ Department of Applied Mathematics, University of Rajshahi, Rajshahi, Bangladesh
}

Email address:

azad267@gmail.com (M. A. K. Azad), abubakarpk_ru@yahoo.com (M. A. Bkar Pk), am.math.1970@gmail.com (A. Malek)

To cite this article:

M. Abul Kalam Azad, M. Abu Bkar Pk, Abdul Malek. Effect of Chemical Reaction on Statistical Theory of Dusty Fluid MHD Turbulent Flow for Certain Variables at Three- Point Distribution Functions. Science Journal of Applied Mathematics and Statistics.

Vol. 3, No. 3, 2015, pp. 75-98. doi: 10.11648/j.sjams.20150303.13

\begin{abstract}
In this paper, an attempt is made to study the three-point distribution functions in dusty fluid MHD turbulent flow for simultaneous velocity, magnetic temperature and concentration fields in a first order chemical reaction. It has been discussed the various properties of constructed distribution functions. From beginning to end out the study, the transport equation for three-point distribution functions in dusty fluid MHD turbulent flow undergoing a first order chemical reaction has been obtained. The obtained equation is compared with the first equation of BBGKY hierarchy of equations and the closure difficulty is to be removed as in the case of ordinary turbulence.
\end{abstract}

Keywords: First Order Chemical Reaction, Dusty Fluid, MHD Turbulent Flow, Three-Point Distribution Functions, Magnetic, Temperature, Concentration

\section{Introduction}

Nowadays dusty viscous fluid motion has developed rapidly which plays an important role in turbulent flow. The phenomenon of this type occurs in the movement of dustladen air, in problem of fluidization, in the use of dust in a gas cooling system and in the sedimentation problem of tidal rivers. The behavior of dust particle in a turbulent flow depends on the concentration of the particles and the size of the particles with respect to the scale of turbulent fluid. In molecular kinetic theory in physics, a particle's distribution function is a function of several variables. Particle distribution functions are used in plasma physics to describe wave-particle interactions and velocity-space instabilities. Distribution functions are also used in fluid mechanics, statistical mechanics and nuclear physics. Also a first-order reaction is defined a reaction that proceeds at a rate that depends linearly only on one reactant concentration. In the past, several authors discussed the distribution functions in the statistical theory of turbulence such as Hopf (1952), Kraichanan (1956), Edward (1964), and Herring (1965). Further Lundgren (1967) derived a hierarchy of coupled equations for multi-point turbulence velocity distribution functions, which resemble with BBGKY hierarchy of equations of Ta-You (1966) in the kinetic theory of gasses. Bigler (1976) gave the hypothesis that in turbulent flames, the thermo chemical quantities can be related locally to few scalars and considered the probability density function of these scalars. Kishore (1978) studied the Distributions functions in the statistical theory of MHD turbulence of an incompressible fluid. Pope (1979) studied the statistical theory of turbulence flames. Pope (1981) derived the transport equation for the joint probability density function of velocity and scalars in turbulent flow. Kollman and Janicka (1982) derived the transport equation for the probability density function of a scalar in turbulent shear flow and considered a closure model based on gradient - flux model. Kishore and Singh (1984) derived the transport equation for the bivariate joint distribution function of velocity and temperature in turbulent flow. Also Kishore and Singh (1985) have been derived the transport equation for the joint distribution function of velocity, temperature and concentration in convective turbulent flow.

Dixit and Upadhyay (1989) considered the distribution functions in the statistical theory of MHD turbulence of an incompressible fluid in the presence of the coriolis force. Sarker and Kishore (1991) discussed the distribution functions in the statistical theory of convective MHD 
turbulence of an incompressible fluid. Also Sarker and Kishore (1999) studied the distribution functions in the statistical theory of convective MHD turbulence of mixture of a miscible incompressible fluid. Sarker and Islam (2002) studied the distribution functions in the statistical theory of convective MHD turbulence of an incompressible fluid in a rotating system. In the continuation of the above researchers, Azad and Sarker(2003) considered the decay of MHD turbulence before the final period for the case of multi-point and multi-time in presence of dust particle, Azad and Sarker (2004a) discussed statistical theory of certain distribution functions in MHD turbulence in a rotating system in presence of dust particles, Sarker and Azad (2004b) studied the decay of MHD turbulence before the final period for the case of multi-point and multi-time in a rotating system. Azad and Sarker(2008) considered the decaying of temperature fluctuations in homogeneous turbulence before the final period for the case of multi- point and multi- time in a rotating system in presence of dust particles. Azad and Sarker(2009a) had measured the decay of temperature fluctuations in MHD turbulence before the final period in a rotating system.

In the next the following authors considered $1^{\text {st }}$ order reaction in their works. Sarker and Azad (2006), Islam and Sarker (2007) studied distribution functions in the statistical theory of MHD turbulence for velocity and concentration undergoing a first order reaction. In their continuation the following authors is also included first order reaction in their works., Azad et al $(2009 b, 2009 c)$ studied the first order reactant in Magneto-hydrodynamic turbulence before the final Period of decay with dust particles and rotating System. Aziz et al (2009d, 2010c) discussed the first order reactant in Magneto- hydrodynamic turbulence before the final period of decay for the case of multi-point and multi-time taking rotating system and dust particles. Aziz et al (2010a, 2010b) studied the statistical theory of certain distribution functions in MHD turbulent flow undergoing a first order reaction in presence of dust particles and rotating system separately. Azad et al (2011) studied the statistical theory of certain distribution Functions in MHD turbulent flow for velocity and concentration undergoing a first order reaction in a rotating system. Azad et al (2012) derived the transport equatoin for the joint distribution function of velocity, temperature and concentration in convective tubulent flow in presence of dust particles. Molla et al (2012) studied the decay of temperature fluctuations in homogeneous turbulenc before the final period in a rotating system. Bkar Pk. et al (2012) studed the First-order reactant in homogeneou dusty fluid turbulence prior to the ultimate phase of decay for fourpoint correlation in a rotating system. Azad and Mumtahinah(2013) studied the decay of temperature fluctuations in dusty fluid homogeneous turbulence prior to final period. Bkar Pk. et al (2013a,2013b) discussed the firstorder reactant in homogeneous turbulence prior to the ultimate phase of decay for four-point correlation with dust particle and rotating system. Bkar Pk.et al (2013,2013c, 2013d) studied the decay of MHD turbulence before the final period for four- point correlation in a rotating system and dust particles. Very recent Azad et al (2014a) derived the transport equations of three point distribution functions in MHD turbulent flow for velocity, magnetic temperature and concentration, Azad and Nazmul (2014b) considered the transport equations of three point distribution functions in MHD turbulent flow for velocity, magnetic temperature and concentration in a rotating system, Nazmul and Azad (2014) studied the transport equations of three-point distribution functions in MHD turbulent flow for velocity, magnetic temperature and concentration in presence of dust particles. Azad and Mumtahinah (2014) further has been studied the transport equatoin for the joint distribution functions in convective tubulent flow in presence of dust particles undergoing a first order reaction. Very recently, Bkar Pk. et al (2015) considering the effects of first-order reactant on MHD turbulence at four-point correlation. Azad et al (2015) derived a transport equation for the joint distribution functions of certain variables in convective dusty fluid turbulent flow in a rotating system undergoing a first order reaction. Bkar $\mathrm{Pk}$, et al (2015a) studied the 4-point correlations of dusty fluid MHD turbulent flow in a $1^{\text {st }}$ order reaction. Azad et al (2015a) considered the 3-Point distribution functions in the statistical theory in MHD turbulent flow for velocity, Magnetic temperature and concentration undergoing a first order reaction.

In this paper, the main purpose is to study the statistical theory of 3-point distribution function for simultaneous velocity, magnetic, temperature, concentration fields in MHD turbulence in presence of dust particles undergoing a first order reaction. Throughout the study, the transport equations for evolution of distribution functions have been derived and various properties of the distribution function have been discussed. The obtained three-point transport equation is compared with the first equation of BBGKY hierarchy of equations and the closure difficulty is to be removed as in the case of ordinary turbulence.

\section{Material and Methods}

\subsection{Basic Equations}

The equations of motion and continuity for viscous incompressible dusty fluid MHD turbulent with constant reaction rate, the diffusion equations for the temperature and concentration are given by

$$
\begin{gathered}
\frac{\partial u_{\alpha}}{\partial t}+\frac{\partial}{\partial x_{\beta}}\left(u_{\alpha} u_{\beta}-h_{\alpha} h_{\beta}\right)=-\frac{1}{4 \pi} \frac{\partial}{\partial x_{\alpha}} \int\left[\frac{\partial u_{\alpha}^{\prime}}{\partial x_{\beta}^{\prime}} \frac{\partial u_{\beta}^{\prime}}{\partial x_{\alpha}^{\prime}}-\frac{\partial h_{\alpha}^{\prime}}{\partial x_{\beta}^{\prime}} \frac{\partial h_{\beta}^{\prime}}{\partial x_{\alpha}^{\prime}}\right] \frac{d \bar{x}^{\prime}}{\left|\bar{x}^{\prime}-\bar{x}\right|}+\nu \nabla^{2} u_{\alpha} \\
+f\left(u_{\alpha}-v_{\alpha}\right) \\
\frac{\partial h_{\alpha}}{\partial t}+\frac{\partial}{\partial x_{\beta}}\left(h_{\alpha} u_{\beta}-u_{\alpha} h_{\beta}\right)=\lambda \nabla^{2} h_{\alpha} \\
\frac{\partial \theta}{\partial t}+u_{\beta} \frac{\partial \theta}{\partial x_{\beta}}=\gamma \nabla^{2} \theta
\end{gathered}
$$




$$
\frac{\partial c}{\partial t}+u_{\beta} \frac{\partial c}{\partial x_{\beta}}=D \nabla^{2} c-R c
$$

with

$$
\frac{\partial u_{\alpha}}{\partial x_{\alpha}}=\frac{\partial v_{\alpha}}{\partial x_{\alpha}}=\frac{\partial h_{\alpha}}{\partial x_{\alpha}}=0,
$$

where $u_{\alpha}(x, t), \alpha$ - component of turbulent velocity; $h_{\alpha}(x, t), \alpha$ - component of magnetic field; $\theta(x, t)$, temperature fluctuation; $\mathrm{c}$, concentration of contaminants; $\mathrm{v} \alpha$, dust particle velocity; $f=\frac{K N}{\rho}$, dimension of frequency; $\mathrm{N}$, constant number of density of the dust particle; $w(\hat{x}, t)=P / \rho+\frac{1}{2}|\vec{h}|^{2}+\frac{1}{2}|\hat{\Omega} \times \hat{x}|^{2}$, total pressure; $P(\hat{x}, t)$, hydrodynamic pressure; $\rho$, fluid density; $v$, Kinematic viscosity; $\lambda=(4 \pi \mu \sigma)^{-1}$, magnetic diffusivity; $\gamma=\frac{k_{T}}{\rho c_{p}}$, thermal diffusivity; $c_{p}$, specific heat at constant pressure; $\mathrm{k}_{\mathrm{T}}$, thermal conductivity; $\sigma$, electrical conductivity; $\mu$, magnetic permeability; D, diffusivity co-efficient for contaminants; $\mathrm{R}$, constant reaction rate.

The repeated suffices are assumed over the values 1, 2 and 3 and unrepeated suffices may take any of these values. In the whole process $\mathrm{u}, \mathrm{h}$ and $\mathrm{x}$ are the vector quantities.

\subsection{Formulation of the Problem}

It has considered that the turbulence and the concentration fields are homogeneous, the chemical reaction and the local mass transfer have no effect on the velocity field and the reaction rate and the diffusivity are constant. It is also considered a large ensemble of identical fluids in which each member is an infinite incompressible reacting and heat conducting fluid in turbulent state. The fluid velocity $u$, Alfven velocity $h$, temperature $\theta$ and concentration $\mathrm{c}$ are randomly distributed functions of position and time and satisfy their field. Different members of ensemble are subjected to different initial conditions and the aim is to find out a way by which we can determine the ensemble averages at the initial time.

Certain microscopic properties of conducting fluids such as total energy, total pressure, stress tensor which are nothing but ensemble averages at a particular time can be determined with the help of the distribution functions (defined as the averaged distribution functions with the help of Dirac deltafunctions). The present aim is to construct a joint distribution function for its evolution of three-point distribution functions in dusty fluid MHD turbulent flow undergoing first order reaction, study its properties and derive a transport equation for the joint distribution function of velocity, temperature and concentration in dusty fluid MHD turbulent flow undergoing a first order reaction.

\subsection{Distribution Function in MHD Turbulence and Their Properties}

In MHD turbulence, it is considered that the fluid velocity $\mathrm{u}$, Alfven velocity $\mathrm{h}$, temperature $\theta$ and concentration $\mathrm{c}$ at each point of the flow field. Corresponding to each point of the flow field, there are four measurable characteristics represent by the four variables by $\mathrm{v}, \mathrm{g}, \varphi$ and $\psi$ and denote the pairs of these variables at the points $\bar{x}^{(1)}, \bar{x}^{(2)},-----, \bar{x}^{(n)} \quad$ as $\quad\left(\bar{v}^{(1)}, \bar{g}^{(1)}, \varphi^{(1)}, \psi^{(1)}\right)$, $\left(\bar{v}^{(2)}, \bar{g}^{(2)}, \varphi^{(2)}, \psi^{(2)}\right),---\left(\bar{v}^{(n)}, \bar{g}^{(n)}, \varphi^{(n)}, \psi^{(n)}\right)$ at a fixed instant of time.

It is possible that the same pair may be occurred more than once; therefore, it simplifies the problem by an assumption that the distribution is discrete (in the sense that no pairs occur more than once). Symbolically we can express the bivariate distribution as

$$
\begin{gathered}
\left\{\left(\bar{v}^{(1)}, \bar{g}^{(1)}, \varphi^{(1)}, \psi^{(1)}\right) ;\right. \\
\left.\left(\bar{v}^{(2)}, \bar{g}^{(2)}, \varphi^{(2)}, \psi^{(2)}\right) ;------\left(\bar{v}^{(n)}, \bar{g}^{(n)}, \varphi^{(n)}, \psi^{(n)}\right)\right\}
\end{gathered}
$$

Instead of considering discrete points in the flow field, if it is considered the continuous distribution of the variables $\bar{v}, \bar{g}, \varphi$ and $\psi$ over the entire flow field, statistically behavior of the fluid may be described by the distribution function $F(\bar{v}, \bar{g}, \varphi, \psi)$ which is normalized so that

$$
\int F(\bar{v}, \bar{g}, \varphi, \psi) d \bar{v} d \bar{g} d \varphi d \psi=1
$$

where the integration ranges over all the possible values of $\mathrm{v}$, $\mathrm{g}, \varphi$ and $\psi$. We shall make use of the same normalization condition for the discrete distributions also.

The distribution functions of the above quantities can be defined in terms of Dirac delta function.

The one-point distribution function $F_{1}^{(1)}\left(v^{(1)}, g^{(1)}, \varphi^{(1)}, \psi^{(1)}\right)$,defined so that $F_{1}^{(1)}\left(v^{(1)}, g^{(1)}, \varphi^{(1)}, \psi^{(1)}\right) d v^{(1)} d g^{(1)} d \varphi^{(1)} d \psi^{(1)}$ is the probability that the fluid velocity, Alfven velocity, temperature and concentration at a time $t$ are in the element $d v^{(1)}$ about $v^{(1)}$, $\mathrm{dg}^{(1)}$ about $\mathrm{g}^{(1)}, \mathrm{d} \varphi^{(1)}$ about $\varphi^{(1)}$ and $\mathrm{d} \psi^{(1)}$ about $\psi^{(1)}$ respectively and is given by

$$
F_{1}^{(1)}\left(v^{(1)}, g^{(1)}, \varphi^{(1)}, \psi^{(1)}\right)=\left\langle\delta\left(u^{(1)}-v^{(1)}\right) \delta\left(h^{(1)}-g^{(1)}\right) \delta\left(\theta^{(1)}-\varphi^{(1)}\right) \delta\left(c^{(1)}-\psi^{(1)}\right)\right\rangle
$$

where $\delta$ is the Dirac delta-function defined as

$$
\int \delta(\bar{u}-\bar{v}) d \bar{v}= \begin{cases}1 & \text { at the point } \bar{u}=\bar{v} \\ 0 & \text { elsewhere }\end{cases}
$$


Two-point distribution function is given by

$$
F_{2}^{(1,2)}=\left\langle\delta\left(u^{(1)}-v^{(1)}\right) \delta\left(h^{(1)}-g^{(1)}\right) \delta\left(\theta^{(1)}-\varphi^{(1)}\right) \delta\left(c^{(1)}-\psi^{(1)}\right) \delta\left(u^{(2)}-v^{(2)}\right) \delta\left(h^{(2)}-g^{(2)}\right) \delta\left(\theta^{(2)}-\varphi^{(2)}\right) \delta\left(c^{(2)}-\psi^{(2)}\right)\right\rangle
$$

and three point distribution function is given by

$$
\begin{aligned}
F_{3}^{(1,2,3)}= & \left\langle\delta\left(u^{(1)}-v^{(1)}\right) \delta\left(h^{(1)}-g^{(1)}\right) \delta\left(\theta^{(1)}-\varphi^{(1)}\right) \delta\left(c^{(1)}-\psi^{(1)}\right) \delta\left(u^{(2)}-v^{(2)}\right) \delta\left(h^{(2)}-g^{(2)}\right)\right. \\
& \left.\times \delta\left(\theta^{(2)}-\varphi^{(2)}\right) \delta\left(c^{(2)}-\psi^{(2)}\right) \delta\left(u^{(3)}-v^{(3)}\right) \delta\left(h^{(3)}-g^{(3)}\right) \delta\left(\theta^{(3)}-\varphi^{(3)}\right) \delta\left(c^{(3)}-\psi^{(3)}\right)\right\rangle
\end{aligned}
$$

Similarly, we can define an infinite numbers of multi-point distribution functions $\mathrm{F}_{4}^{(1,2,3,4)}, \mathrm{F}_{5}^{(1,2,3,4,5)}$ and so on. The following properties of the constructed distribution functions can be deduced from the above definitions:

(A) Reduction Properties:

Integration with respect to pair of variables at one-point lowers the order of distribution function by one. For example,

$$
\begin{gathered}
\int F_{1}^{(1)} d v^{(1)} d g^{(1)} d \varphi^{(1)} d \psi^{(1)}=1, \\
\int F_{2}^{(1,2)} d v^{(2)} d g^{(2)} d \varphi^{(2)} d \psi^{(2)}=F_{1}^{(1)}, \\
\int F_{3}^{(1,2,3)} d v^{(3)} d g^{(3)} d \varphi^{(3)} d \psi^{(3)}=F_{2}^{(1,2)} \\
\int F_{2}^{(1,2)} d v^{(2)}=\left\langle\delta\left(u^{(1)}-v^{(1)}\right) \delta\left(h^{(1)}-g^{(1)}\right) \delta\left(\theta^{(1)}-\varphi^{(1)}\right) \delta\right. \\
\text { (B) Separation Properties: } \\
\text { If two points are far apart from each other in the flow field, } \\
\text { the pairs of variables at these points are statistically }
\end{gathered}
$$$$
\int F_{2}^{(1,2)} d v^{(2)}=\left\langle\delta\left(u^{(1)}-v^{(1)}\right) \delta\left(h^{(1)}-g^{(1)}\right) \delta\left(\theta^{(1)}-\varphi^{(1)}\right) \delta\left(c^{(1)}-\psi^{(1)}\right) \delta\left(h^{(2)}-g^{(2)}\right) \delta\left(\theta^{(2)}-\varphi^{(2)}\right) \delta\left(c^{(2)}-\psi^{(2)}\right)\right\rangle
$$

And so on. Also the integration with respect to any one of the variables, reduces the number of Delta-functions from the distribution function by one as

$$
\begin{aligned}
& \int F_{1}^{(1)} d v^{(1)}=\left\langle\delta\left(h^{(1)}-g^{(1)}\right) \delta\left(\theta^{(1)}-\varphi^{(1)}\right) \delta\left(c^{(1)}-\psi^{(1)}\right)\right\rangle, \\
& \int F_{1}^{(1)} d g^{(1)}=\left\langle\delta\left(u^{(1)}-v^{(1)}\right) \delta\left(\theta^{(1)}-\varphi^{(1)}\right) \delta\left(c^{(1)}-\psi^{(1)}\right)\right\rangle, \\
& \int F_{1}^{(1)} d \varphi^{(1)}=\left\langle\delta\left(u^{(1)}-v^{(1)}\right) \delta\left(h^{(1)}-g^{(1)}\right) \delta\left(c^{(1)}-\psi^{(1)}\right)\right\rangle,
\end{aligned}
$$

and

(C) Co-incidence Properties:

(B) Separation Properties: independent of each other i.e.,

$\lim$

$$
\left|\vec{x}^{(2)} \rightarrow \bar{x}^{(1)}\right| \rightarrow \infty \quad F_{2}^{(1,2)}=F_{1}^{(1)} F_{1}^{(2)}
$$

and similarly,

$\lim$

$\left|\bar{x}^{(3)} \rightarrow \bar{x}^{(2)}\right| \rightarrow \infty \quad F_{3}^{(1,2,3)}=F_{2}^{(1,2)} F_{1}^{(3)}$ etc.

$\lim$

$$
\left|\bar{x}^{(2)} \rightarrow \bar{x}^{(1)}\right| \rightarrow \infty \int F_{2}^{(1,2)}=F_{1}^{(1)} \delta\left(v^{(2)}-v^{(1)}\right) \delta\left(g^{(2)}-g^{(1)}\right) \delta\left(\varphi^{(2)}-\varphi^{(1)}\right) \delta\left(\psi^{(2)}-\psi^{(1)}\right)
$$

Similarly,

$\lim$

$\left|\bar{x}^{(3)} \rightarrow \bar{x}^{(2)}\right| \rightarrow \infty \int F_{3}^{(1,2,3)}=F_{2}^{(1,2)} \delta\left(v^{(3)}-v^{(1)}\right) \delta\left(g^{(3)}-g^{(1)}\right) \delta\left(\varphi^{(3)}-\varphi^{(1)}\right) \delta\left(\psi^{(3)}-\psi^{(1)}\right)$ etc.

(D) Symmetric Conditions:

$$
F_{n}^{(1,2, r,-------s,-----n)}=F_{n}^{(1,2,-----s,---r,---n)} .
$$

(E) Incompressibility Conditions: (i) $\int \frac{\partial F_{n}^{(1,2,---n)}}{\partial x_{\alpha}^{(r)}} v_{\alpha}^{(r)} d \bar{v}^{(r)} d \bar{h}^{(r)}=0$

(ii) $\int \frac{\partial F_{n}^{(1,2,---n)}}{\partial x_{\alpha}^{(r)}} h_{\alpha}^{(r)} d \bar{v}^{(r)} d \bar{h}^{(r)}=0$ components at these points should be obviously the same that is $\mathrm{F}_{2}^{(1,2)}$ must be zero.

Thus $\bar{v}^{(2)}=\bar{v}^{(1)}, g^{(2)}=g^{(1)}, \varphi^{(2)}=\varphi^{(1)}$ and $\psi^{(2)}=\psi^{(1)}$, but F2 $(1,2)$ must also have the property.

$$
\int F_{2}^{(1,2)} d v^{(2)} d g^{(2)} d \varphi^{(2)} d \psi^{(2)}=F_{1}^{(1)}
$$

and hence it follows that
When two points coincide in the flow field, the 


\subsection{Continuity Equation in Terms of Distribution Functions}

The continuity equations can be easily expressed in terms of distribution functions. An infinite number of continuity equations can be derived for the convective MHD turbulent flow and are obtained directly by using $\operatorname{div} u=0$

Taking ensemble average of equation (5), we get

$$
\begin{array}{r}
0=\left\langle\frac{\partial u_{\alpha}^{(1)}}{\partial x_{\alpha}^{(1)}}\right\rangle=\left\langle\frac{\partial}{\partial x_{\alpha}^{(1)}} u_{\alpha}^{(1)} \int F_{1}^{(1)} d v^{(1)} d g^{(1)} d \varphi^{(1)} d \psi^{(1)}\right\rangle=\frac{\partial}{\partial x_{\alpha}^{(1)}}\left\langle u_{\alpha}^{(1)} \int F_{1}^{(1)} d v^{(1)} d g^{(1)} d \varphi^{(1)} d \psi^{(1)}\right\rangle \\
=\frac{\partial}{\partial x_{\alpha}^{(1)}} \int\left\langle u_{\alpha}^{(1)}\right\rangle\left\langle F_{1}^{(1)}\right\rangle d v^{(1)} d g^{(1)} d \varphi^{(1)} d \psi^{(1)}=\frac{\partial}{\partial x_{\alpha}^{(1)}} \int v_{\alpha}^{(1)} F_{1}^{(1)} d v^{(1)} d g^{(1)} d \varphi^{(1)} d \psi^{(1)}=\int \frac{\partial F_{1}^{(1)}}{\partial x_{\alpha}^{(1)}} v_{\alpha}^{(1)} d v^{(1)} d g^{(1)} d \varphi^{(1)} d \psi^{(1)} \quad(9)
\end{array}
$$

and similarly,

$$
0=\int \frac{\partial F_{1}^{(1)}}{\partial x_{\alpha}^{(1)}} g_{\alpha}^{(1)} d v^{(1)} d g^{(1)} d \varphi^{(1)} d \psi^{(1)}
$$

involved.

For second-order continuity equations, if we multiply the continuity equation by

$$
\delta\left(u^{(2)}-v^{(2)}\right) \delta\left(h^{(2)}-g^{(2)}\right) \delta\left(\theta^{(2)}-\varphi^{(2)}\right) \delta\left(c^{(2)}-\psi^{(2)}\right)
$$

and if we take the ensemble average, we obtain

Equation (15) and (16) are the first order continuity equations in which only one point distribution function is

$$
\begin{gathered}
o=\left\langle\delta\left(u^{(2)}-v^{(2)}\right) \delta\left(h^{(2)}-g^{(2)}\right) \delta\left(\theta^{(2)}-\varphi^{(2)}\right) \delta\left(c^{(2)}-\psi^{(2)}\right) \frac{\partial u_{\alpha}^{(1)}}{\partial x_{\alpha}^{(1)}}\right\rangle \\
=\frac{\partial}{\partial x_{\alpha}^{(1)}}\left\langle\delta\left(u^{(2)}-v^{(2)}\right) \delta\left(h^{(2)}-g^{(2)}\right) \delta\left(\theta^{(2)}-\varphi^{(2)}\right) \delta\left(c^{(2)}-\psi^{(2)}\right) u_{\alpha}^{(1)}\right\rangle \\
=\frac{\partial}{\partial x_{\alpha}^{(1)}}\left[\int \left\langleu_{\alpha}^{(1)} \delta\left(u^{(1)}-v^{(1)}\right) \delta\left(h^{(1)}-g^{(1)}\right) \delta\left(\theta^{(1)}-\varphi^{(1)}\right) \delta\left(c^{(1)}-\psi^{(1)}\right)\right.\right. \\
\left.\left.\times \delta\left(u^{(2)}-v^{(2)}\right) \delta\left(h^{(2)}-g^{(2)}\right) \delta\left(\theta^{(2)}-\varphi^{(2)}\right) \delta\left(c^{(2)}-\psi^{(2)}\right)\right\rangle d v^{(1)} d g^{(1)} d \varphi^{(1)} d \psi^{(1)}\right]=\frac{\partial}{\partial x_{\alpha}^{(1)}} \int v_{\alpha}^{(1)} F_{2}^{(1,2)} d v^{(1)} d g^{(1)} d \varphi^{(1)} d \psi^{(1)}
\end{gathered}
$$

and similarly,

and

$$
o=\frac{\partial}{\partial x_{\alpha}^{(1)}} \int g_{\alpha}^{(1)} F_{2}^{(1,2)} d v^{(1)} d g^{(1)} d \varphi^{(1)} d \psi^{(1)}
$$

The Nth - order continuity equations are

$$
\begin{aligned}
& o=\frac{\partial}{\partial x_{\alpha}^{(1)}} \int v_{\alpha}^{(1)} F_{N}^{(1,2,---, N)} d v^{(1)} d g^{(1)} d \varphi^{(1)} d \psi^{(1)} \text { i.e. } \\
& \frac{\partial}{\partial x_{\alpha}^{(r)}}\left(v_{\alpha}^{(r)} F_{N}^{(1,2, \ldots \ldots \ldots, r . N)} d v^{(r)} d g^{(r)} d \varphi^{(r)} d \psi^{(r)}\right)=\frac{\partial}{\partial x_{\alpha}^{(s)}} \int v_{\alpha}^{(s)} F_{N}^{(1,2, \ldots, r, s, \ldots . N)} d v^{(s)} d g^{(s)} d \varphi^{(s)} d \psi^{(s)}
\end{aligned}
$$

The continuity equations are symmetric in their arguments

$$
o=\frac{\partial}{\partial x_{\alpha}^{(1)}} \int g_{\alpha}^{(1)} F_{N}^{(1,2, \ldots \ldots ., N)} d v^{(1)} d g^{(1)} d \varphi^{(1)} d \psi^{(1)}
$$

Since the divergence property is an important property and it is easily verified by the use of the property of distribution function as

$$
\frac{\partial}{\partial x_{\alpha}^{(1)}} \int v_{\alpha}^{(1)} F_{1}^{(1)} d v^{(1)} d g^{(1)} d \varphi^{(1)} d \psi^{(1)} \frac{\partial}{\partial x_{\alpha}^{(1)}}\left\langle u_{\alpha}^{(1)}\right\rangle=\left\langle\frac{\partial u_{\alpha}^{(1)}}{\partial x_{\alpha}^{(1)}}\right\rangle=o
$$

and all the properties of the distribution function obtained in section (4) can also be verified.

\subsection{Equations for Three-Point Distribution Function $F_{3}^{(1,2.3)}$}

It shall make use of equations (1) - (4) to convert these into a set of equations for the variation of the distribution function with time. This, in fact, is done by making use of the definitions of the constructed distribution functions, differentiating them partially with respect to time, making some suitable operations on the right-hand side of the equation so obtained and lastly replacing the time derivative of $u, h, \theta$ and $\mathrm{c}$ from the equations (1) - (4).

Differentiating equation (8) with respect to time and using equation (1) - (4), we get, 


$$
\begin{aligned}
& \frac{\partial F_{3}^{(1,2,3)}}{\partial t}=\frac{\partial}{\partial t}\left\langle\delta\left(u^{(1)}-v^{(1)}\right) \delta\left(h^{(1)}-g^{(1)}\right) \delta\left(\theta^{(1)}-\varphi^{(1)}\right) \delta\left(c^{(1)}-\psi^{(1)}\right) \delta\left(u^{(2)}-v^{(2)}\right) \delta\left(h^{(2)}-g^{(2)}\right)\right. \\
& \left.\delta\left(\theta^{(2)}-\varphi^{(2)}\right) \delta\left(c^{(2)}-\psi^{(2)}\right) \delta\left(u^{(3)}-v^{(3)}\right) \delta\left(h^{(3)}-g^{(3)}\right) \delta\left(\theta^{(3)}-\varphi^{(3)}\right) \delta\left(c^{(3)}-\psi^{(3)}\right)\right\rangle \\
& =\left\langle\delta\left(h^{(1)}-g^{(1)}\right) \delta\left(\theta^{(1)}-\varphi^{(1)}\right) \delta\left(c^{(1)}-\psi^{(1)}\right) \delta\left(u^{(2)}-v^{(2)}\right) \delta\left(h^{(2)}-g^{(2)}\right) \delta\left(\theta^{(2)}-\varphi^{(2)}\right)\right. \\
& \left.\delta\left(c^{(2)}-\psi^{(2)}\right) \delta\left(u^{(3)}-v^{(3)}\right) \delta\left(h^{(3)}-g^{(3)}\right) \delta\left(\theta^{(3)}-\varphi^{(3)}\right) \delta\left(c^{(3)}-\psi^{(3)}\right) \frac{\partial}{\partial t} \delta\left(u^{(1)}-v^{(1)}\right)\right\rangle \\
& +\left\langle\delta\left(u^{(1)}-v^{(1)}\right) \delta\left(\theta^{(1)}-\varphi^{(1)}\right) \delta\left(c^{(1)}-\psi^{(1)}\right) \delta\left(u^{(2)}-v^{(2)}\right) \delta\left(h^{(2)}-g^{(2)}\right) \delta\left(\theta^{(2)}-\varphi^{(2)}\right)\right. \\
& \left.\delta\left(c^{(2)}-\psi^{(2)}\right) \delta\left(u^{(3)}-v^{(3)}\right) \delta\left(h^{(3)}-g^{(3)}\right) \delta\left(\theta^{(3)}-\varphi^{(3)}\right) \delta\left(c^{(3)}-\psi^{(3)}\right) \frac{\partial}{\partial t} \delta\left(h^{(1)}-g^{(1)}\right)\right\rangle \\
& +\left\langle\delta\left(u^{(1)}-v^{(1)}\right) \delta\left(h^{(1)}-g^{(1)}\right) \delta\left(c^{(1)}-\psi^{(1)}\right) \delta\left(u^{(2)}-v^{(2)}\right) \delta\left(h^{(2)}-g^{(2)}\right) \delta\left(\theta^{(2)}-\varphi^{(2)}\right)\right. \\
& \left.\delta\left(c^{(2)}-\psi^{(2)}\right) \delta\left(u^{(3)}-v^{(3)}\right) \delta\left(h^{(3)}-g^{(3)}\right) \delta\left(\theta^{(3)}-\varphi^{(3)}\right) \delta\left(c^{(3)}-\psi^{(3)}\right) \frac{\partial}{\partial t} \delta\left(\theta^{(1)}-\varphi^{(1)}\right)\right\rangle \\
& +\left\langle\delta\left(u^{(1)}-v^{(1)}\right) \delta\left(h^{(1)}-g^{(1)}\right) \delta\left(\theta^{(1)}-\varphi^{(1)}\right) \delta\left(u^{(2)}-v^{(2)}\right) \delta\left(h^{(2)}-g^{(2)}\right) \delta\left(\theta^{(2)}-\varphi^{(2)}\right)\right. \\
& \left.\delta\left(c^{(2)}-\psi^{(2)}\right) \delta\left(u^{(3)}-v^{(3)}\right) \delta\left(h^{(3)}-g^{(3)}\right) \delta\left(\theta^{(3)}-\varphi^{(3)}\right) \delta\left(c^{(3)}-\psi^{(3)}\right) \frac{\partial}{\partial t} \delta\left(c^{(1)}-\psi^{(1)}\right)\right\rangle \\
& +\left\langle\delta\left(u^{(1)}-v^{(1)}\right) \delta\left(h^{(1)}-g^{(1)}\right) \delta\left(\theta^{(1)}-\varphi^{(1)}\right) \delta\left(c^{(1)}-\psi^{(1)}\right) \delta\left(h^{(2)}-g^{(2)}\right) \delta\left(\theta^{(2)}-\varphi^{(2)}\right)\right. \\
& \left.\delta\left(c^{(2)}-\psi^{(2)}\right) \delta\left(u^{(3)}-v^{(3)}\right) \delta\left(h^{(3)}-g^{(3)}\right) \delta\left(\theta^{(3)}-\varphi^{(3)}\right) \delta\left(c^{(3)}-\psi^{(3)}\right) \frac{\partial}{\partial t} \delta\left(u^{(2)}-v^{(2)}\right)\right\rangle \\
& +\left\langle\delta\left(u^{(1)}-v^{(1)}\right) \delta\left(h^{(1)}-g^{(1)}\right) \delta\left(\theta^{(1)}-\varphi^{(1)}\right) \delta\left(c^{(1)}-\psi^{(1)}\right) \delta\left(u^{(2)}-v^{(2)}\right) \delta\left(\theta^{(2)}-\varphi^{(2)}\right)\right. \\
& \left.\delta\left(c^{(2)}-\psi^{(2)}\right) \delta\left(u^{(3)}-v^{(3)}\right) \delta\left(h^{(3)}-g^{(3)}\right) \delta\left(\theta^{(3)}-\varphi^{(3)}\right) \delta\left(c^{(3)}-\psi^{(3)}\right) \frac{\partial}{\partial t} \delta\left(h^{(2)}-g^{(2)}\right)\right\rangle \\
& +\left\langle\delta\left(u^{(1)}-v^{(1)}\right) \delta\left(h^{(1)}-g^{(1)}\right) \delta\left(\theta^{(1)}-\varphi^{(1)}\right) \delta\left(c^{(1)}-\psi^{(1)}\right) \delta\left(u^{(2)}-v^{(2)}\right) \delta\left(h^{(2)}-g^{(2)}\right)\right. \\
& \left.\delta\left(c^{(2)}-\psi^{(2)}\right) \delta\left(u^{(3)}-v^{(3)}\right) \delta\left(h^{(3)}-g^{(3)}\right) \delta\left(\theta^{(3)}-\varphi^{(3)}\right) \delta\left(c^{(3)}-\psi^{(3)}\right) \frac{\partial}{\partial t} \delta\left(\theta^{(2)}-\varphi^{(2)}\right)\right\rangle \\
& +\left\langle\delta\left(u^{(1)}-v^{(1)}\right) \delta\left(h^{(1)}-g^{(1)}\right) \delta\left(\theta^{(1)}-\varphi^{(1)}\right) \delta\left(c^{(1)}-\psi^{(1)}\right) \delta\left(u^{(2)}-v^{(2)}\right) \delta\left(h^{(2)}-g^{(2)}\right)\right. \\
& \left.\delta\left(\theta^{(2)}-\varphi^{(2)}\right) \delta\left(u^{(3)}-v^{(3)}\right) \delta\left(h^{(3)}-g^{(3)}\right) \delta\left(\theta^{(3)}-\varphi^{(3)}\right) \delta\left(c^{(3)}-\psi^{(3)}\right) \frac{\partial}{\partial t} \delta\left(c^{(2)}-\psi^{(2)}\right)\right\rangle \\
& +\left\langle\delta\left(u^{(1)}-v^{(1)}\right) \delta\left(h^{(1)}-g^{(1)}\right) \delta\left(\theta^{(1)}-\varphi^{(1)}\right) \delta\left(c^{(1)}-\psi^{(1)}\right) \delta\left(u^{(2)}-v^{(2)}\right) \delta\left(h^{(2)}-g^{(2)}\right)\right. \\
& \left.\delta\left(\theta^{(2)}-\varphi^{(2)}\right) \delta\left(c^{(2)}-\psi^{(2)}\right) \delta\left(h^{(3)}-g^{(3)}\right) \delta\left(\theta^{(3)}-\varphi^{(3)}\right) \delta\left(c^{(3)}-\psi^{(3)}\right) \frac{\partial}{\partial t} \delta\left(u^{(3)}-v^{(3)}\right)\right\rangle \\
& +\left\langle\delta\left(u^{(1)}-v^{(1)}\right) \delta\left(h^{(1)}-g^{(1)}\right) \delta\left(\theta^{(1)}-\varphi^{(1)}\right) \delta\left(c^{(1)}-\psi^{(1)}\right) \delta\left(u^{(2)}-v^{(2)}\right) \delta\left(h^{(2)}-g^{(2)}\right)\right. \\
& \left.\delta\left(\theta^{(2)}-\varphi^{(2)}\right) \delta\left(c^{(2)}-\psi^{(2)}\right) \delta\left(u^{(3)}-v^{(3)}\right) \delta\left(\theta^{(3)}-\varphi^{(3)}\right) \delta\left(c^{(3)}-\psi^{(3)}\right) \frac{\partial}{\partial t} \delta\left(h^{(3)}-g^{(3)}\right)\right\rangle \\
& +\left\langle\delta\left(u^{(1)}-v^{(1)}\right) \delta\left(h^{(1)}-g^{(1)}\right) \delta\left(\theta^{(1)}-\varphi^{(1)}\right) \delta\left(c^{(1)}-\psi^{(1)}\right) \delta\left(u^{(2)}-v^{(2)}\right) \delta\left(h^{(2)}-g^{(2)}\right)\right. \\
& \left.\delta\left(\theta^{(2)}-\varphi^{(2)}\right) \delta\left(c^{(2)}-\psi^{(2)}\right) \delta\left(u^{(3)}-v^{(3)}\right) \delta\left(h^{(3)}-g^{(3)}\right) \delta\left(c^{(3)}-\psi^{(3)}\right) \frac{\partial}{\partial t} \delta\left(\theta^{(3)}-\varphi^{(3)}\right)\right\rangle \\
& +\left\langle\delta\left(u^{(1)}-v^{(1)}\right) \delta\left(h^{(1)}-g^{(1)}\right) \delta\left(\theta^{(1)}-\varphi^{(1)}\right) \delta\left(c^{(1)}-\psi^{(1)}\right) \delta\left(u^{(2)}-v^{(2)}\right) \delta\left(h^{(2)}-g^{(2)}\right)\right. \\
& \left.\delta\left(\theta^{(2)}-\varphi^{(2)}\right) \delta\left(c^{(2)}-\psi^{(2)}\right) \delta\left(u^{(3)}-v^{(3)}\right) \delta\left(h^{(3)}-g^{(3)}\right) \delta\left(\theta^{(3)}-\varphi^{(3)}\right) \frac{\partial}{\partial t} \delta\left(c^{(3)}-\psi^{(3)}\right)\right\rangle \\
& =\left\langle-\delta\left(h^{(1)}-g^{(1)}\right) \delta\left(\theta^{(1)}-\varphi^{(1)}\right) \delta\left(c^{(1)}-\psi^{(1)}\right) \delta\left(u^{(2)}-v^{(2)}\right) \delta\left(h^{(2)}-g^{(2)}\right) \delta\left(\theta^{(2)}-\varphi^{(2)}\right)\right. \\
& \left.\delta\left(c^{(2)}-\psi^{(2)}\right) \delta\left(u^{(3)}-v^{(3)}\right) \delta\left(h^{(3)}-g^{(3)}\right) \delta\left(\theta^{(3)}-\varphi^{(3)}\right) \delta\left(c^{(3)}-\psi^{(3)}\right) \frac{\partial u^{(1)}}{\partial t} \frac{\partial}{\partial v^{(1)}} \delta\left(u^{(1)}-v^{(1)}\right)\right\rangle
\end{aligned}
$$




$$
\begin{aligned}
& +\left\langle-\delta\left(u^{(1)}-v^{(1)}\right) \delta\left(\theta^{(1)}-\varphi^{(1)}\right) \delta\left(c^{(1)}-\psi^{(1)}\right) \delta\left(u^{(2)}-v^{(2)}\right) \delta\left(h^{(2)}-g^{(2)}\right) \delta\left(\theta^{(2)}-\varphi^{(2)}\right) \delta\left(c^{(2)}-\psi^{(2)}\right)\right. \\
& \left.\delta\left(u^{(3)}-v^{(3)}\right) \delta\left(h^{(3)}-g^{(3)}\right) \delta\left(\theta^{(3)}-\varphi^{(3)}\right) \delta\left(c^{(3)}-\psi^{(3)}\right) \frac{\partial h^{(1)}}{\partial t} \frac{\partial}{\partial g^{(1)}} \delta\left(h^{(1)}-g^{(1)}\right)\right\rangle \\
& +\left\langle-\delta\left(u^{(1)}-v^{(1)}\right) \delta\left(h^{(1)}-g^{(1)}\right) \delta\left(c^{(1)}-\psi^{(1)}\right) \delta\left(u^{(2)}-v^{(2)}\right) \delta\left(h^{(2)}-g^{(2)}\right) \delta\left(\theta^{(2)}-\varphi^{(2)}\right) \delta\left(c^{(2)}-\psi^{(2)}\right)\right. \\
& \left.\delta\left(u^{(3)}-v^{(3)}\right) \delta\left(h^{(3)}-g^{(3)}\right) \delta\left(\theta^{(3)}-\varphi^{(3)}\right) \delta\left(c^{(3)}-\psi^{(3)}\right) \frac{\partial \theta^{(1)}}{\partial t} \frac{\partial}{\partial \varphi^{(1)}} \delta\left(\theta^{(1)}-\varphi^{(1)}\right)\right\rangle \\
& +\left\langle-\delta\left(u^{(1)}-v^{(1)}\right) \delta\left(h^{(1)}-g^{(1)}\right) \delta\left(\theta^{(1)}-\varphi^{(1)}\right) \delta\left(u^{(2)}-v^{(2)}\right) \delta\left(h^{(2)}-g^{(2)}\right) \delta\left(\theta^{(2)}-\varphi^{(2)}\right) \delta\left(c^{(2)}-\psi^{(2)}\right)\right. \\
& \left.\delta\left(u^{(3)}-v^{(3)}\right) \delta\left(h^{(3)}-g^{(3)}\right) \delta\left(\theta^{(3)}-\varphi^{(3)}\right) \delta\left(c^{(3)}-\psi^{(3)}\right) \frac{\partial c^{(1)}}{\partial t} \frac{\partial}{\partial \psi^{(1)}} \delta\left(c^{(1)}-\psi^{(1)}\right)\right\rangle \\
& +\left\langle-\delta\left(u^{(1)}-v^{(1)}\right) \delta\left(h^{(1)}-g^{(1)}\right) \delta\left(\theta^{(1)}-\varphi^{(1)}\right) \delta\left(c^{(1)}-\psi^{(1)}\right) \delta\left(h^{(2)}-g^{(2)}\right) \delta\left(\theta^{(2)}-\varphi^{(2)}\right) \delta\left(c^{(2)}-\psi^{(2)}\right)\right. \\
& \left.\delta\left(u^{(3)}-v^{(3)}\right) \delta\left(h^{(3)}-g^{(3)}\right) \delta\left(\theta^{(3)}-\varphi^{(3)}\right) \delta\left(c^{(3)}-\psi^{(3)}\right) \frac{\partial u^{(2)}}{\partial t} \frac{\partial}{\partial v^{(2)}} \delta\left(u^{(2)}-v^{(2)}\right)\right\rangle \\
& +\left\langle-\delta\left(u^{(1)}-v^{(1)}\right) \delta\left(h^{(1)}-g^{(1)}\right) \delta\left(\theta^{(1)}-\varphi^{(1)}\right) \delta\left(c^{(1)}-\psi^{(1)}\right) \delta\left(u^{(2)}-v^{(2)}\right) \delta\left(\theta^{(2)}-\varphi^{(2)}\right) \delta\left(c^{(2)}-\psi^{(2)}\right)\right. \\
& \left.\delta\left(u^{(3)}-v^{(3)}\right) \delta\left(h^{(3)}-g^{(3)}\right) \delta\left(\theta^{(3)}-\varphi^{(3)}\right) \delta\left(c^{(3)}-\psi^{(3)}\right) \frac{\partial \theta^{(2)}}{\partial t} \frac{\partial}{\partial \varphi^{(2)}} \delta\left(\theta^{(2)}-\varphi^{(2)}\right)\right\rangle \\
& +\left\langle-\delta\left(u^{(1)}-v^{(1)}\right) \delta\left(h^{(1)}-g^{(1)}\right) \delta\left(\theta^{(1)}-\varphi^{(1)}\right) \delta\left(c^{(1)}-\psi^{(1)}\right) \delta\left(u^{(2)}-v^{(2)}\right) \delta\left(h^{(2)}-g^{(2)}\right) \delta\left(\theta^{(2)}-\varphi^{(2)}\right)\right. \\
& \left.\delta\left(u^{(3)}-v^{(3)}\right) \delta\left(h^{(3)}-g^{(3)}\right) \delta\left(\theta^{(3)}-\varphi^{(3)}\right) \delta\left(c^{(3)}-\psi^{(3)}\right) \frac{\partial c^{(2)}}{\partial t} \frac{\partial}{\partial \psi^{(2)}} \delta\left(c^{(2)}-\psi^{(2)}\right)\right\rangle \\
& +\left\langle-\delta\left(u^{(1)}-v^{(1)}\right) \delta\left(h^{(1)}-g^{(1)}\right) \delta\left(\theta^{(1)}-\varphi^{(1)}\right) \delta\left(c^{(1)}-\psi^{(1)}\right) \delta\left(u^{(2)}-v^{(2)}\right) \delta\left(h^{(2)}-g^{(2)}\right) \delta\left(\theta^{(2)}-\varphi^{(2)}\right)\right. \\
& \left.\delta\left(c^{(2)}-\psi^{(2)}\right) \delta\left(h^{(3)}-g^{(3)}\right) \delta\left(\theta^{(3)}-\varphi^{(3)}\right) \delta\left(c^{(3)}-\psi^{(3)}\right) \frac{\partial u^{(3)}}{\partial t} \frac{\partial}{\partial v^{(3)}} \delta\left(u^{(3)}-v^{(3)}\right)\right\rangle \\
& +\left\langle-\delta\left(u^{(1)}-v^{(1)}\right) \delta\left(h^{(1)}-g^{(1)}\right) \delta\left(\theta^{(1)}-\varphi^{(1)}\right) \delta\left(c^{(1)}-\psi^{(1)}\right) \delta\left(u^{(2)}-v^{(2)}\right) \delta\left(h^{(2)}-g^{(2)}\right) \delta\left(\theta^{(2)}-\varphi^{(2)}\right)\right. \\
& \left.\delta\left(c^{(2)}-\psi^{(2)}\right) \delta\left(u^{(3)}-v^{(3)}\right) \delta\left(\theta^{(3)}-\varphi^{(3)}\right) \delta\left(c^{(3)}-\psi^{(3)}\right) \frac{\partial h^{(3)}}{\partial t} \frac{\partial}{\partial g^{(3)}} \delta\left(h^{(3)}-g^{(3)}\right)\right\rangle \\
& +\left\langle-\delta\left(u^{(1)}-v^{(1)}\right) \delta\left(h^{(1)}-g^{(1)}\right) \delta\left(\theta^{(1)}-\varphi^{(1)}\right) \delta\left(c^{(1)}-\psi^{(1)}\right) \delta\left(u^{(2)}-v^{(2)}\right) \delta\left(h^{(2)}-g^{(2)}\right) \delta\left(\theta^{(2)}-\varphi^{(2)}\right)\right. \\
& \left.\delta\left(c^{(2)}-\psi^{(2)}\right) \delta\left(u^{(3)}-v^{(3)}\right) \delta\left(h^{(3)}-g^{(3)}\right) \delta\left(c^{(3)}-\psi^{(3)}\right) \frac{\partial \theta^{(3)}}{\partial t} \frac{\partial}{\partial \varphi^{(3)}} \delta\left(\theta^{(3)}-\varphi^{(3)}\right)\right\rangle \\
& +\left\langle-\delta\left(u^{(1)}-v^{(1)}\right) \delta\left(h^{(1)}-g^{(1)}\right) \delta\left(\theta^{(1)}-\varphi^{(1)}\right) \delta\left(c^{(1)}-\psi^{(1)}\right) \delta\left(u^{(2)}-v^{(2)}\right) \delta\left(h^{(2)}-g^{(2)}\right) \delta\left(\theta^{(2)}-\varphi^{(2)}\right)\right. \\
& \left.\delta\left(c^{(2)}-\psi^{(2)}\right) \delta\left(u^{(3)}-v^{(3)}\right) \delta\left(h^{(3)}-g^{(3)}\right) \delta\left(\theta^{(3)}-\varphi^{(3)}\right) \frac{\partial c^{(3)}}{\partial t} \frac{\partial}{\partial \psi^{(3)}} \delta\left(c^{(3)}-\psi^{(3)}\right)\right\rangle
\end{aligned}
$$

Using equations (1) to (4), we get from the above equation

$$
\begin{aligned}
& \frac{\partial F_{3}^{(1,2,3)}}{\partial t}=\left\langle-\delta\left(h^{(1)}-g^{(1)}\right) \delta\left(\theta^{(1)}-\varphi^{(1)}\right) \delta\left(c^{(1)}-\psi^{(1)}\right) \delta\left(u^{(2)}-v^{(2)}\right) \delta\left(h^{(2)}-g^{(2)}\right) \delta\left(\theta^{(2)}-\varphi^{(2)}\right)\right. \\
& \delta\left(c^{(2)}-\right.\left.\psi^{(2)}\right) \delta\left(u^{(3)}-v^{(3)}\right) \delta\left(h^{(3)}-g^{(3)}\right) \delta\left(\theta^{(3)}-\varphi^{(3)}\right) \delta\left(c^{(3)}-\psi^{(3)}\right) \\
&\left\{-\frac{\partial}{\partial x_{\beta}^{(1)}}\left(u_{\alpha}^{(1)} u_{\beta}^{(1)}-h_{\alpha}^{(1)} h_{\beta}^{(1)}\right)-\frac{1}{4 \pi} \frac{\partial}{\partial x_{\alpha}^{(1)}} \int\left[\frac{\partial u_{\alpha}^{(1)}}{\partial x_{\beta}^{(1)}} \frac{\partial u_{\beta}^{(1)}}{\partial x_{\alpha}^{(1)}}-\frac{\partial h_{\alpha}^{(1)}}{\partial x_{\beta}^{(1)}} \frac{\partial h_{\beta}^{(1)}}{\partial x_{\alpha}^{(1)}}\right] \frac{d \bar{x}^{\prime \prime \prime}}{\left|\bar{x}^{\prime \prime \prime}-\bar{x}^{n}\right|}\right. \\
&\left.\left.+\gamma \nabla^{2} u_{\alpha}^{(1)}+f\left(u_{\alpha}^{(1)}-v_{\alpha}^{(1)}\right)\right\} \times \frac{\partial}{\partial v_{\alpha}^{(1)}} \delta\left(u^{(1)}-v^{(1)}\right)\right\rangle \\
&+\left\langle-\delta\left(u^{(1)}-v^{(1)}\right) \delta\left(\theta^{(1)}-\varphi^{(1)}\right) \delta\left(c^{(1)}-\psi^{(1)}\right) \delta\left(u^{(2)}-v^{(2)}\right) \delta\left(h^{(2)}-g^{(2)}\right) \delta\left(\theta^{(2)}-\varphi^{(2)}\right)\right. \\
& \delta\left(c^{(2)}-\psi^{(2)}\right) \delta\left(u^{(3)}-v^{(3)}\right) \delta\left(h^{(3)}-g^{(3)}\right) \delta\left(\theta^{(3)}-\varphi^{(3)}\right) \delta\left(c^{(3)}-\psi^{(3)}\right)
\end{aligned}
$$




$$
\begin{aligned}
& \left.\left\{-\frac{\partial}{\partial x_{\beta}^{(1)}}\left(h_{\alpha}^{(1)} u_{\beta}^{(1)}-u_{\alpha}^{(1)} h_{\beta}^{(1)}\right)+\lambda \nabla^{2} h_{\alpha}^{(1)}\right\} \times \frac{\partial}{\partial g_{\alpha}^{(1)}} \delta\left(h^{(1)}-g^{(1)}\right)\right\rangle \\
& +\left\langle-\delta\left(u^{(1)}-v^{(1)}\right) \delta\left(h^{(1)}-g^{(1)}\right) \delta\left(c^{(1)}-\psi^{(1)}\right) \delta\left(u^{(2)}-v^{(2)}\right) \delta\left(h^{(2)}-g^{(2)}\right) \delta\left(\theta^{(2)}-\varphi^{(2)}\right)\right. \\
& \delta\left(c^{(2)}-\psi^{(2)}\right) \delta\left(u^{(3)}-v^{(3)}\right) \delta\left(h^{(3)}-g^{(3)}\right) \delta\left(\theta^{(3)}-\varphi^{(3)}\right) \delta\left(c^{(3)}-\psi^{(3)}\right) \\
& \left.\times\left\{-u_{\beta}^{(1)} \frac{\partial \theta^{(1)}}{\partial x_{\beta}^{(1)}}+\nu^{2} \theta^{(1)}\right\} \times \frac{\partial}{\partial \varphi^{(1)}} \delta\left(\theta^{(1)}-\varphi^{(1)}\right)\right\rangle \\
& +\left\langle-\delta\left(u^{(1)}-v^{(1)}\right) \delta\left(h^{(1)}-g^{(1)}\right) \delta\left(\theta^{(1)}-\varphi^{(1)}\right) \delta\left(u^{(2)}-v^{(2)}\right) \delta\left(h^{(2)}-g^{(2)}\right) \delta\left(\theta^{(2)}-\varphi^{(2)}\right)\right. \\
& \delta\left(c^{(2)}-\psi^{(2)}\right) \delta\left(u^{(3)}-v^{(3)}\right) \delta\left(h^{(3)}-g^{(3)}\right) \delta\left(\theta^{(3)}-\varphi^{(3)}\right) \delta\left(c^{(3)}-\psi^{(3)}\right) \\
& \left.\left\{-u_{\beta}^{(1)} \frac{\partial c^{(1)}}{\partial x_{\beta}^{(1)}}+D \nabla^{2} c^{(1)}-R c^{(1)}\right\} \times \frac{\partial}{\partial \psi^{(1)}} \delta\left(c^{(1)}-\psi^{(1)}\right)\right\rangle \\
& \left\langle-\delta\left(u^{(1)}-v^{(1)}\right) \delta\left(h^{(1)}-g^{(1)}\right) \delta\left(\theta^{(1)}-\varphi^{(1)}\right) \delta\left(c^{(1)}-\psi^{(1)}\right) \delta\left(h^{(2)}-g^{(2)}\right) \delta\left(\theta^{(2)}-\varphi^{(2)}\right)\right. \\
& \delta\left(c^{(2)}-\psi^{(2)}\right) \delta\left(u^{(3)}-v^{(3)}\right) \delta\left(h^{(3)}-g^{(3)}\right) \delta\left(\theta^{(3)}-\varphi^{(3)}\right) \delta\left(c^{(3)}-\psi^{(3)}\right) \\
& \left\{-\frac{\partial}{\partial x_{\beta}^{(2)}}\left(u_{\alpha}^{(2)} u_{\beta}^{(2)}-h_{\alpha}^{(2)} h_{\beta}^{(2)}\right)-\frac{1}{4 \pi} \frac{\partial}{\partial x_{\alpha}^{(2)}} \int\left[\frac{\partial u_{\alpha}^{(2)}}{\partial x_{\beta}^{(2)}} \frac{\partial u_{\beta}^{(2)}}{\partial x_{\alpha}^{(2)}}-\frac{\partial h_{\alpha}^{(2)}}{\partial x_{\beta}^{(2)}} \frac{\partial h_{\beta}^{(2)}}{\partial x_{\alpha}^{(2)}}\right] \frac{d \bar{x}^{\prime \prime \prime}}{\left|\bar{x}^{\prime \prime \prime}-\bar{x}^{\prime}\right|}\right. \\
& \left.\left.+v \nabla^{2} u_{\alpha}^{(2)}+f\left(u_{\alpha}^{(2)}-v_{\alpha}^{(2)}\right)\right\} \times \frac{\partial}{\partial v_{\alpha}^{(2)}} \delta\left(u^{(2)}-v^{(2)}\right)\right\rangle \\
& +\left\langle-\delta\left(u^{(1)}-v^{(1)}\right) \delta\left(h^{(1)}-g^{(1)}\right) \delta\left(\theta^{(1)}-\varphi^{(1)}\right) \delta\left(c^{(1)}-\psi^{(1)}\right) \delta\left(u^{(2)}-v^{(2)}\right) \delta\left(\theta^{(2)}-\varphi^{(2)}\right)\right. \\
& \delta\left(c^{(2)}-\psi^{(2)}\right) \delta\left(u^{(3)}-v^{(3)}\right) \delta\left(h^{(3)}-g^{(3)}\right) \delta\left(\theta^{(3)}-\varphi^{(3)}\right) \delta\left(c^{(3)}-\psi^{(3)}\right) \\
& +\left\langle-\delta\left(u^{(1)}-v^{(1)}\right) \delta\left(h^{(1)}-g^{(1)}\right) \delta\left(\theta^{(1)}-\varphi^{(1)}\right) \delta\left(c^{(1)}-\psi^{(1)}\right) \delta\left(u^{(2)}-v^{(2)}\right) \delta\left(h^{(2)}-g^{(2)}\right)\right. \\
& \delta\left(\theta^{(2)}-\varphi^{(2)}\right) \delta\left(u^{(3)}-v^{(3)}\right) \delta\left(h^{(3)}-g^{(3)}\right) \delta\left(\theta^{(3)}-\varphi^{(3)}\right) \delta\left(c^{(3)}-\psi^{(3)}\right) \\
& \left.\left\{-u_{\beta}^{(2)} \frac{\partial c^{(2)}}{\partial x_{\beta}^{(2)}}+D \nabla^{2} c^{(2)}-R c^{(2)}\right\} \frac{\partial}{\partial \psi^{(2)}} \delta\left(c^{(2)}-\psi^{(2)}\right)\right\rangle \\
& \left.\left\{-\frac{\partial}{\partial x_{\beta}^{(2)}}\left(h_{\alpha}^{(2)} u_{\beta}^{(2)}-u_{\alpha}^{(2)} h_{\beta}^{(2)}\right)+\lambda \nabla^{2} h_{\alpha}^{(2)}\right\} \frac{\partial}{\partial g_{\alpha}^{(2)}} \delta\left(h^{(2)}-g^{(2)}\right)\right\rangle \\
& +\left\langle-\delta\left(u^{(1)}-v^{(1)}\right) \delta\left(h^{(1)}-g^{(1)}\right) \delta\left(\theta^{(1)}-\varphi^{(1)}\right) \delta\left(c^{(1)}-\psi^{(1)}\right) \delta\left(u^{(2)}-v^{(2)}\right) \delta\left(h^{(2)}-g^{(2)}\right)\right. \\
& \delta\left(c^{(2)}-\psi^{(2)}\right) \delta\left(u^{(3)}-v^{(3)}\right) \delta\left(h^{(3)}-g^{(3)}\right) \delta\left(\theta^{(3)}-\varphi^{(3)}\right) \delta\left(c^{(3)}-\psi^{(3)}\right) \\
& \left.\times\left\{-u_{\beta}^{(2)} \frac{\partial \theta^{(2)}}{\partial x_{\beta}^{(2)}}+\gamma^{2} \theta^{(2)}\right\} \times \frac{\partial}{\partial \varphi^{(2)}} \delta\left(\theta^{(2)}-\varphi^{(2)}\right)\right\rangle \\
& \left\langle-\delta\left(u^{(1)}-v^{(1)}\right) \delta\left(h^{(1)}-g^{(1)}\right) \delta\left(\theta^{(1)}-\varphi^{(1)}\right) \delta\left(c^{(1)}-\psi^{(1)}\right) \delta\left(u^{(2)}-v^{(2)}\right) \delta\left(h^{(2)}-g^{(2)}\right)\right. \\
& \delta\left(\theta^{(2)}-\varphi^{(2)}\right) \delta\left(c^{(2)}-\psi^{(2)}\right) \delta\left(h^{(3)}-g^{(3)}\right) \delta\left(\theta^{(3)}-\varphi^{(3)}\right) \delta\left(c^{(3)}-\psi^{(3)}\right) \\
& \left\{-\frac{\partial}{\partial x_{\beta}^{(3)}}\left(u_{\alpha}^{(3)} u_{\beta}^{(3)}-h_{\alpha}^{(3)} h_{\beta}^{(3)}\right)-\frac{1}{4 \pi} \frac{\partial}{\partial x_{\alpha}^{(3)}} \int\left[\frac{\partial u_{\alpha}^{(3)}}{\partial x_{\beta}^{(3)}} \frac{\partial u_{\beta}^{(3)}}{\partial x_{\alpha}^{(3)}}-\frac{\partial h_{\alpha}^{(3)}}{\partial x_{\beta}^{(3)}} \frac{\partial h_{\beta}^{(3)}}{\partial x_{\alpha}^{(3)}}\right] \frac{d \bar{x}^{\prime \prime \prime}}{\left|\bar{x}^{\prime \prime \prime}-\bar{x}^{\prime \prime}\right|}\right. \\
& \left.\left.+v \nabla^{2} u_{\alpha}^{(3)}+f\left(u_{\alpha}^{(3)}-v_{\alpha}^{(3)}\right)\right\} \times \frac{\partial}{\partial v_{\alpha}^{(3)}} \delta\left(u^{(3)}-v^{(3)}\right)\right\rangle \\
& +\left\langle-\delta\left(u^{(1)}-v^{(1)}\right) \delta\left(h^{(1)}-g^{(1)}\right) \delta\left(\theta^{(1)}-\varphi^{(1)}\right) \delta\left(c^{(1)}-\psi^{(1)}\right) \delta\left(u^{(2)}-v^{(2)}\right) \delta\left(h^{(2)}-g^{(2)}\right)\right. \\
& \delta\left(\theta^{(2)}-\varphi^{(2)}\right) \delta\left(c^{(2)}-\psi^{(2)}\right) \delta\left(u^{(3)}-v^{(3)}\right) \delta\left(\theta^{(3)}-\varphi^{(3)}\right) \delta\left(c^{(3)}-\psi^{(3)}\right)
\end{aligned}
$$




$$
\begin{aligned}
& \left.\left\{-\frac{\partial}{\partial x_{\beta}^{(3)}}\left(h_{\alpha}^{(3)} u_{\beta}^{(3)}-u_{\alpha}^{(3)} h_{\beta}^{(3)}\right)+\lambda \nabla^{2} h_{\alpha}^{(3)}\right\} \frac{\partial}{\partial g_{\alpha}^{(3)}} \delta\left(h^{(3)}-g^{(3)}\right)\right\rangle \\
& +\left\langle-\delta\left(u^{(1)}-v^{(1)}\right) \delta\left(h^{(1)}-g^{(1)}\right) \delta\left(\theta^{(1)}-\varphi^{(1)}\right) \delta\left(c^{(1)}-\psi^{(1)}\right) \delta\left(u^{(2)}-v^{(2)}\right) \delta\left(h^{(2)}-g^{(2)}\right)\right. \\
& \delta\left(\theta^{(2)}-\varphi^{(2)}\right) \delta\left(c^{(2)}-\psi^{(2)}\right) \delta\left(u^{(3)}-v^{(3)}\right) \delta\left(h^{(3)}-g^{(3)}\right) \delta\left(c^{(3)}-\psi^{(3)}\right) \\
& \left.\times\left\{-u_{\beta}^{(3)} \frac{\partial \theta^{(3)}}{\partial x_{\beta}^{(3)}}+\gamma \nabla^{2} \theta^{(3)}\right\} \times \frac{\partial}{\partial \varphi^{(3)}} \delta\left(\theta^{(3)}-\varphi^{(3)}\right)\right\rangle \\
& +\left\langle-\delta\left(u^{(1)}-v^{(1)}\right) \delta\left(h^{(1)}-g^{(1)}\right) \delta\left(\theta^{(1)}-\varphi^{(1)}\right) \delta\left(c^{(1)}-\psi^{(1)}\right) \delta\left(u^{(2)}-v^{(2)}\right) \delta\left(h^{(2)}-g^{(2)}\right)\right. \\
& \delta\left(\theta^{(2)}-\varphi^{(2)}\right) \delta\left(c^{(2)}-\psi^{(2)}\right) \delta\left(u^{(3)}-v^{(3)}\right) \delta\left(h^{(3)}-g^{(3)}\right) \delta\left(\theta^{(3)}-\varphi^{(3)}\right) \\
& \left.\left\{-u_{\beta}^{(3)} \frac{\partial c^{(3)}}{\partial x_{\beta}^{(3)}}+D \nabla^{2} c^{(3)}-R c^{(3)}\right\} \frac{\partial}{\partial \psi^{(3)}} \delta\left(c^{(3)}-\psi^{(3)}\right)\right\rangle \\
& =\left\langle\delta\left(h^{(1)}-g^{(1)}\right) \delta\left(\theta^{(1)}-\varphi^{(1)}\right) \delta\left(c^{(1)}-\psi^{(1)}\right) \delta\left(u^{(2)}-v^{(2)}\right) \delta\left(h^{(2)}-g^{(2)}\right) \delta\left(\theta^{(2)}-\varphi^{(2)}\right) \delta\left(c^{(2)}-\psi^{(2)}\right)\right. \\
& \left.\delta\left(u^{(3)}-v^{(3)}\right) \delta\left(h^{(3)}-g^{(3)}\right) \delta\left(\theta^{(3)}-\varphi^{(3)}\right) \delta\left(c^{(3)}-\psi^{(3)}\right) \times \frac{\partial u_{\alpha}^{(1)} u_{\beta}^{(1)}}{\partial x_{\beta}^{(1)}} \frac{\partial}{\partial v_{\alpha}^{(1)}} \delta\left(u^{(1)}-v^{(1)}\right)\right\rangle \\
& +\left\langle-\delta\left(h^{(1)}-g^{(1)}\right) \delta\left(\theta^{(1)}-\varphi^{(1)}\right) \delta\left(c^{(1)}-\psi^{(1)}\right) \delta\left(u^{(2)}-v^{(2)}\right) \delta\left(h^{(2)}-g^{(2)}\right) \delta\left(\theta^{(2)}-\varphi^{(2)}\right) \delta\left(c^{(2)}-\psi^{(2)}\right)\right. \\
& \left.\delta\left(u^{(3)}-v^{(3)}\right) \delta\left(h^{(3)}-g^{(3)}\right) \delta\left(\theta^{(3)}-\varphi^{(3)}\right) \delta\left(c^{(3)}-\psi^{(3)}\right) \times \frac{\partial h_{\alpha}^{(1)} h_{\beta}^{(1)}}{\partial x_{\beta}^{(1)}} \frac{\partial}{\partial v_{\alpha}^{(1)}} \delta\left(u^{(1)}-v^{(1)}\right)\right\rangle \\
& +\left\langle\delta\left(h^{(1)}-g^{(1)}\right) \delta\left(\theta^{(1)}-\varphi^{(1)}\right) \delta\left(c^{(1)}-\psi^{(1)}\right) \delta\left(u^{(2)}-v^{(2)}\right) \delta\left(h^{(2)}-g^{(2)}\right) \delta\left(\theta^{(2)}-\varphi^{(2)}\right) \delta\left(c^{(2)}-\psi^{(2)}\right)\right. \\
& \delta\left(u^{(3)}-v^{(3)}\right) \delta\left(h^{(3)}-g^{(3)}\right) \delta\left(\theta^{(3)}-\varphi^{(3)}\right) \delta\left(c^{(3)}-\psi^{(3)}\right) \times \frac{1}{4 \pi} \frac{\partial}{\partial x_{\alpha}^{(1)}} \int\left[\frac{\partial u_{\alpha}^{(1)}}{\partial x_{\beta}^{(1)}} \frac{\partial u_{\beta}^{(1)}}{\partial x_{\alpha}^{(1)}}-\frac{\partial h_{\alpha}^{(1)}}{\partial x_{\beta}^{(1)}} \frac{\partial h_{\beta}^{(1)}}{\partial x_{\alpha}^{(1)}}\right] \\
& \left.\times \frac{d \bar{x}^{\prime \prime \prime}}{\left|\bar{x}^{\prime \prime \prime}-\bar{x}\right|} \frac{\partial}{\partial v_{\alpha}^{(1)}} \delta\left(u^{(1)}-v^{(1)}\right)\right\rangle+\left\langle-\delta\left(h^{(1)}-g^{(1)}\right) \delta\left(\theta^{(1)}-\varphi^{(1)}\right) \delta\left(c^{(1)}-\psi^{(1)}\right) \delta\left(u^{(2)}-v^{(2)}\right) \delta\left(h^{(2)}-g^{(2)}\right)\right. \\
& \left.\delta\left(\theta^{(2)}-\varphi^{(2)}\right) \delta\left(c^{(2)}-\psi^{(2)}\right) \delta\left(u^{(3)}-v^{(3)}\right) \delta\left(h^{(3)}-g^{(3)}\right) \delta\left(\theta^{(3)}-\varphi^{(3)}\right) \delta\left(c^{(3)}-\psi^{(3)}\right) \times v \nabla^{2} u_{\alpha}^{(1)} \frac{\partial}{\partial v_{\alpha}^{(1)}} \delta\left(u^{(1)}-v^{(1)}\right)\right\rangle \\
& +\left\langle-\delta\left(h^{(1)}-g^{(1)}\right) \delta\left(\theta^{(1)}-\varphi^{(1)}\right) \delta\left(c^{(1)}-\psi^{(1)}\right) \delta\left(u^{(2)}-v^{(2)}\right) \delta\left(h^{(2)}-g^{(2)}\right) \delta\left(\theta^{(2)}-\varphi^{(2)}\right) \delta\left(c^{(2)}-\psi^{(2)}\right)\right. \\
& \left.\delta\left(u^{(3)}-v^{(3)}\right) \delta\left(h^{(3)}-g^{(3)}\right) \delta\left(\theta^{(3)}-\varphi^{(3)}\right) \delta\left(c^{(3)}-\psi^{(3)}\right) \times f\left(u_{\alpha}^{(1)}-v_{\alpha}^{(1)}\right) \frac{\partial}{\partial v_{\alpha}^{(1)}} \delta\left(u^{(1)}-v^{(1)}\right)\right\rangle \\
& +\left\langle\delta\left(u^{(1)}-v^{(1)}\right) \delta\left(\theta^{(1)}-\varphi^{(1)}\right) \delta\left(c^{(1)}-\psi^{(1)}\right) \delta\left(u^{(2)}-v^{(2)}\right) \delta\left(h^{(2)}-g^{(2)}\right) \delta\left(\theta^{(2)}-\varphi^{(2)}\right) \delta\left(c^{(2)}-\psi^{(2)}\right)\right. \\
& \left.\delta\left(u^{(3)}-v^{(3)}\right) \delta\left(h^{(3)}-g^{(3)}\right) \delta\left(\theta^{(3)}-\varphi^{(3)}\right) \delta\left(c^{(3)}-\psi^{(3)}\right) \times \frac{\partial h_{\alpha}^{(1)} u_{\beta}^{(1)}}{\partial x_{\beta}^{(1)}} \frac{\partial}{\partial g_{\alpha}^{(1)}} \delta\left(h^{(1)}-g^{(1)}\right)\right\rangle \\
& +\left\langle-\delta\left(u^{(1)}-v^{(1)}\right) \delta\left(\theta^{(1)}-\varphi^{(1)}\right) \delta\left(c^{(1)}-\psi^{(1)}\right) \delta\left(u^{(2)}-v^{(2)}\right) \delta\left(h^{(2)}-g^{(2)}\right) \delta\left(\theta^{(2)}-\varphi^{(2)}\right) \delta\left(c^{(2)}-\psi^{(2)}\right)\right. \\
& \left.\delta\left(u^{(3)}-v^{(3)}\right) \delta\left(h^{(3)}-g^{(3)}\right) \delta\left(\theta^{(3)}-\varphi^{(3)}\right) \delta\left(c^{(3)}-\psi^{(3)}\right) \times \frac{\partial u_{\alpha}^{(1)} h_{\beta}^{(1)}}{\partial x_{\beta}^{(1)}} \frac{\partial}{\partial g_{\alpha}^{(1)}} \delta\left(h^{(1)}-g^{(1)}\right)\right\rangle \\
& +\left\langle-\delta\left(u^{(1)}-v^{(1)}\right) \delta\left(\theta^{(1)}-\varphi^{(1)}\right) \delta\left(c^{(1)}-\psi^{(1)}\right) \delta\left(u^{(2)}-v^{(2)}\right) \delta\left(h^{(2)}-g^{(2)}\right) \delta\left(\theta^{(2)}-\varphi^{(2)}\right) \delta\left(c^{(2)}-\psi^{(2)}\right)\right. \\
& \left.\delta\left(u^{(3)}-v^{(3)}\right) \delta\left(h^{(3)}-g^{(3)}\right) \delta\left(\theta^{(3)}-\varphi^{(3)}\right) \delta\left(c^{(3)}-\psi^{(3)}\right) \times \lambda \nabla^{2} h_{\alpha}^{(1)} \frac{\partial}{\partial g_{\alpha}^{(1)}} \delta\left(h^{(1)}-g^{(1)}\right)\right\rangle \\
& +\left\langle\delta\left(u^{(1)}-v^{(1)}\right) \delta\left(h^{(1)}-g^{(1)}\right) \delta\left(c^{(1)}-\psi^{(1)}\right) \delta\left(u^{(2)}-v^{(2)}\right) \delta\left(h^{(2)}-g^{(2)}\right) \delta\left(\theta^{(2)}-\varphi^{(2)}\right) \delta\left(c^{(2)}-\psi^{(2)}\right)\right. \\
& \left.\delta\left(u^{(3)}-v^{(3)}\right) \delta\left(h^{(3)}-g^{(3)}\right) \delta\left(\theta^{(3)}-\varphi^{(3)}\right) \delta\left(c^{(3)}-\psi^{(3)}\right) \times u_{\beta}^{(1)} \frac{\partial \theta^{(1)}}{\partial x_{\beta}^{(1)}} \frac{\partial}{\partial \varphi^{(1)}} \delta\left(\theta^{(1)}-\varphi^{(1)}\right)\right\rangle
\end{aligned}
$$




$$
\begin{aligned}
& +\left\langle-\delta\left(u^{(1)}-v^{(1)}\right) \delta\left(h^{(1)}-g^{(1)}\right) \delta\left(c^{(1)}-\psi^{(1)}\right) \delta\left(u^{(2)}-v^{(2)}\right) \delta\left(h^{(2)}-g^{(2)}\right) \delta\left(\theta^{(2)}-\varphi^{(2)}\right) \delta\left(c^{(2)}-\psi^{(2)}\right)\right. \\
& \left.\delta\left(u^{(3)}-v^{(3)}\right) \delta\left(h^{(3)}-g^{(3)}\right) \delta\left(\theta^{(3)}-\varphi^{(3)}\right) \delta\left(c^{(3)}-\psi^{(3)}\right) \times \nu^{2} \theta^{(1)} \frac{\partial}{\partial \varphi^{(1)}} \delta\left(\theta^{(1)}-\varphi^{(1)}\right)\right\rangle \\
& +\left\langle\delta\left(u^{(1)}-v^{(1)}\right) \delta\left(h^{(1)}-g^{(1)}\right) \delta\left(\theta^{(1)}-\varphi^{(1)}\right) \delta\left(u^{(2)}-v^{(2)}\right) \delta\left(h^{(2)}-g^{(2)}\right) \delta\left(\theta^{(2)}-\varphi^{(2)}\right) \delta\left(c^{(2)}-\psi^{(2)}\right)\right. \\
& \left.\delta\left(u^{(3)}-v^{(3)}\right) \delta\left(h^{(3)}-g^{(3)}\right) \delta\left(\theta^{(3)}-\varphi^{(3)}\right) \delta\left(c^{(3)}-\psi^{(3)}\right) \times u_{\beta}^{(1)} \frac{\partial c^{(1)}}{\partial x_{\beta}^{(1)}} \frac{\partial}{\partial \psi^{(1)}} \delta\left(c^{(1)}-\psi^{(1)}\right)\right\rangle \\
& +\left\langle-\delta\left(u^{(1)}-v^{(1)}\right) \delta\left(h^{(1)}-g^{(1)}\right) \delta\left(\theta^{(1)}-\varphi^{(1)}\right) \delta\left(u^{(2)}-v^{(2)}\right) \delta\left(h^{(2)}-g^{(2)}\right) \delta\left(\theta^{(2)}-\varphi^{(2)}\right) \delta\left(c^{(2)}-\psi^{(2)}\right)\right. \\
& \left.\delta\left(u^{(3)}-v^{(3)}\right) \delta\left(h^{(3)}-g^{(3)}\right) \delta\left(\theta^{(3)}-\varphi^{(3)}\right) \delta\left(c^{(3)}-\psi^{(3)}\right) \times D \nabla^{2} c^{(1)} \frac{\partial}{\partial \psi^{(1)}} \delta\left(c^{(1)}-\psi^{(1)}\right)\right\rangle \\
& +\delta\left(u^{(1)}-v^{(1)}\right) \delta\left(h^{(1)}-g^{(1)}\right) \delta\left(\theta^{(1)}-\varphi^{(1)}\right) \delta\left(u^{(2)}-v^{(2)}\right) \delta\left(h^{(2)}-g^{(2)}\right) \delta\left(\theta^{(2)}-\varphi^{(2)}\right) \delta\left(c^{(2)}-\psi^{(2)}\right) \\
& \left.\delta\left(u^{(3)}-v^{(3)}\right) \delta\left(h^{(3)}-g^{(3)}\right) \delta\left(\theta^{(3)}-\varphi^{(3)}\right) \delta\left(c^{(3)}-\psi^{(3)}\right) \times R c^{(1)} \frac{\partial}{\partial \psi^{(1)}} \delta\left(c^{(1)}-\psi^{(1)}\right)\right\rangle \\
& +\left\langle\delta\left(u^{(1)}-v^{(1)}\right) \delta\left(h^{(1)}-g^{(1)}\right) \delta\left(\theta^{(1)}-\varphi^{(1)}\right) \delta\left(c^{(1)}-\psi^{(1)}\right) \delta\left(h^{(2)}-g^{(2)}\right) \delta\left(\theta^{(2)}-\varphi^{(2)}\right) \delta\left(c^{(2)}-\psi^{(2)}\right)\right. \\
& \left.\delta\left(u^{(3)}-v^{(3)}\right) \delta\left(h^{(3)}-g^{(3)}\right) \delta\left(\theta^{(3)}-\varphi^{(3)}\right) \delta\left(c^{(3)}-\psi^{(3)}\right) \times \frac{\partial u_{\alpha}^{(2)} u_{\beta}^{(2)}}{\partial x_{\beta}^{(2)}} \frac{\partial}{\partial v_{\alpha}^{(2)}} \delta\left(u^{(2)}-v^{(2)}\right)\right\rangle \\
& +\left\langle-\delta\left(u^{(1)}-v^{(1)}\right) \delta\left(h^{(1)}-g^{(1)}\right) \delta\left(\theta^{(1)}-\varphi^{(1)}\right) \delta\left(c^{(1)}-\psi^{(1)}\right) \delta\left(h^{(2)}-g^{(2)}\right) \delta\left(\theta^{(2)}-\varphi^{(2)}\right) \delta\left(c^{(2)}-\psi^{(2)}\right)\right. \\
& \left.\delta\left(u^{(3)}-v^{(3)}\right) \delta\left(h^{(3)}-g^{(3)}\right) \delta\left(\theta^{(3)}-\varphi^{(3)}\right) \delta\left(c^{(3)}-\psi^{(3)}\right) \times \frac{\partial h_{\alpha}^{(2)} h_{\beta}^{(2)}}{\partial x_{\beta}^{(2)}} \frac{\partial}{\partial v_{\alpha}^{(2)}} \delta\left(u^{(2)}-v^{(2)}\right)\right\rangle \\
& +\left\langle\delta\left(u^{(1)}-v^{(1)}\right) \delta\left(h^{(1)}-g^{(1)}\right) \delta\left(\theta^{(1)}-\varphi^{(1)}\right) \delta\left(c^{(1)}-\psi^{(1)}\right) \delta\left(h^{(2)}-g^{(2)}\right) \delta\left(\theta^{(2)}-\varphi^{(2)}\right) \delta\left(c^{(2)}-\psi^{(2)}\right)\right. \\
& \delta\left(u^{(3)}-v^{(3)}\right) \delta\left(h^{(3)}-g^{(3)}\right) \delta\left(\theta^{(3)}-\varphi^{(3)}\right) \delta\left(c^{(3)}-\psi^{(3)}\right) \times \frac{1}{4 \pi} \frac{\partial}{\partial x_{\alpha}^{(2)}} \int\left[\frac{\partial u_{\alpha}^{(2)}}{\partial x_{\beta}^{(2)}} \frac{\partial u_{\beta}^{(2)}}{\partial x_{\alpha}^{(2)}}-\frac{\partial h_{\alpha}^{(2)}}{\partial x_{\beta}^{(2)}} \frac{\partial h_{\beta}^{(2)}}{\partial x_{\alpha}^{(2)}}\right] \\
& \left.\times \frac{d \bar{x}^{\prime \prime \prime}}{\left|\bar{x}^{\prime \prime \prime}-\bar{x}^{\prime}\right|} \frac{\partial}{\partial v_{\alpha}^{(2)}} \delta\left(u^{(2)}-v^{(2)}\right)\right\rangle \\
& +\left\langle-\delta\left(u^{(1)}-v^{(1)}\right) \delta\left(h^{(1)}-g^{(1)}\right) \delta\left(\theta^{(1)}-\varphi^{(1)}\right) \delta\left(c^{(1)}-\psi^{(1)}\right) \delta\left(h^{(2)}-g^{(2)}\right) \delta\left(\theta^{(2)}-\varphi^{(2)}\right) \delta\left(c^{(2)}-\psi^{(2)}\right)\right. \\
& \left.\delta\left(u^{(3)}-v^{(3)}\right) \delta\left(h^{(3)}-g^{(3)}\right) \delta\left(\theta^{(3)}-\varphi^{(3)}\right) \delta\left(c^{(3)}-\psi^{(3)}\right) \times v \nabla^{2} u_{\alpha}^{(2)} \frac{\partial}{\partial v_{\alpha}^{(2)}} \delta\left(u^{(2)}-v^{(2)}\right)\right\rangle \\
& +\left\langle-\delta\left(u^{(1)}-v^{(1)}\right) \delta\left(h^{(1)}-g^{(1)}\right) \delta\left(\theta^{(1)}-\varphi^{(1)}\right) \delta\left(c^{(1)}-\psi^{(1)}\right) \delta\left(h^{(2)}-g^{(2)}\right) \delta\left(\theta^{(2)}-\varphi^{(2)}\right) \delta\left(c^{(2)}-\psi^{(2)}\right)\right. \\
& \left.\delta\left(u^{(3)}-v^{(3)}\right) \delta\left(h^{(3)}-g^{(3)}\right) \delta\left(\theta^{(3)}-\varphi^{(3)}\right) \delta\left(c^{(3)}-\psi^{(3)}\right) \times f\left(u_{\alpha}^{(2)}-v_{\alpha}^{(2)}\right) \frac{\partial}{\partial v_{\alpha}^{(2)}} \delta\left(u^{(2)}-v^{(2)}\right)\right\rangle \\
& +\left\langle\delta\left(u^{(1)}-v^{(1)}\right) \delta\left(h^{(1)}-g^{(1)}\right) \delta\left(\theta^{(1)}-\varphi^{(1)}\right) \delta\left(c^{(1)}-\psi^{(1)}\right) \delta\left(u^{(2)}-v^{(2)}\right) \delta\left(\theta^{(2)}-\varphi^{(2)}\right) \delta\left(c^{(2)}-\psi^{(2)}\right)\right. \\
& \left.\delta\left(u^{(3)}-v^{(3)}\right) \delta\left(h^{(3)}-g^{(3)}\right) \delta\left(\theta^{(3)}-\varphi^{(3)}\right) \delta\left(c^{(3)}-\psi^{(3)}\right) \times \frac{\partial h_{\alpha}^{(2)} u_{\beta}^{(2)}}{\partial x_{\beta}^{(2)}} \frac{\partial}{\partial g_{\alpha}^{(2)}} \delta\left(h^{(2)}-g^{(2)}\right)\right\rangle \\
& +\left\langle-\delta\left(u^{(1)}-v^{(1)}\right) \delta\left(h^{(1)}-g^{(1)}\right) \delta\left(\theta^{(1)}-\varphi^{(1)}\right) \delta\left(c^{(1)}-\psi^{(1)}\right) \delta\left(u^{(2)}-v^{(2)}\right) \delta\left(\theta^{(2)}-\varphi^{(2)}\right) \delta\left(c^{(2)}-\psi^{(2)}\right)\right. \\
& \left.\delta\left(u^{(3)}-v^{(3)}\right) \delta\left(h^{(3)}-g^{(3)}\right) \delta\left(\theta^{(3)}-\varphi^{(3)}\right) \delta\left(c^{(3)}-\psi^{(3)}\right) \times \frac{\partial u_{\alpha}^{(2)} h_{\beta}^{(2)}}{\partial x_{\beta}^{(2)}} \frac{\partial}{\partial g_{\alpha}^{(2)}} \delta\left(h^{(2)}-g^{(2)}\right)\right\rangle \\
& +\left\langle-\delta\left(u^{(1)}-v^{(1)}\right) \delta\left(h^{(1)}-g^{(1)}\right) \delta\left(\theta^{(1)}-\varphi^{(1)}\right) \delta\left(c^{(1)}-\psi^{(1)}\right) \delta\left(u^{(2)}-v^{(2)}\right) \delta\left(\theta^{(2)}-\varphi^{(2)}\right) \delta\left(c^{(2)}-\psi^{(2)}\right)\right. \\
& \left.\delta\left(u^{(3)}-v^{(3)}\right) \delta\left(h^{(3)}-g^{(3)}\right) \delta\left(\theta^{(3)}-\varphi^{(3)}\right) \delta\left(c^{(3)}-\psi^{(3)}\right) \times \lambda \nabla^{2} h_{\alpha}^{(2)} \frac{\partial}{\partial g_{\alpha}^{(2)}} \delta\left(h^{(2)}-g^{(2)}\right)\right\rangle
\end{aligned}
$$




$$
\begin{aligned}
& +\left\langle\delta\left(u^{(1)}-v^{(1)}\right) \delta\left(h^{(1)}-g^{(1)}\right) \delta\left(\theta^{(1)}-\varphi^{(1)}\right) \delta\left(c^{(1)}-\psi^{(1)}\right) \delta\left(u^{(2)}-v^{(2)}\right) \delta\left(h^{(2)}-g^{(2)}\right) \delta\left(c^{(2)}-\psi^{(2)}\right)\right. \\
& \left.\delta\left(u^{(3)}-v^{(3)}\right) \delta\left(h^{(3)}-g^{(3)}\right) \delta\left(\theta^{(3)}-\varphi^{(3)}\right) \delta\left(c^{(3)}-\psi^{(3)}\right) \times u_{\beta}^{(2)} \frac{\partial \theta^{(2)}}{\partial x_{\beta}^{(2)}} \frac{\partial}{\partial \varphi^{(2)}} \delta\left(\theta^{(2)}-\varphi^{(2)}\right)\right\rangle \\
& +\left\langle-\delta\left(u^{(1)}-v^{(1)}\right) \delta\left(h^{(1)}-g^{(1)}\right) \delta\left(\theta^{(1)}-\varphi^{(1)}\right) \delta\left(c^{(1)}-\psi^{(1)}\right) \delta\left(u^{(2)}-v^{(2)}\right) \delta\left(h^{(2)}-g^{(2)}\right) \delta\left(c^{(2)}-\psi^{(2)}\right)\right. \\
& \left.\delta\left(u^{(3)}-v^{(3)}\right) \delta\left(h^{(3)}-g^{(3)}\right) \delta\left(\theta^{(3)}-\varphi^{(3)}\right) \delta\left(c^{(3)}-\psi^{(3)}\right) \times \nabla^{2} \theta^{(2)} \frac{\partial}{\partial \varphi^{(2)}} \delta\left(\theta^{(2)}-\varphi^{(2)}\right)\right\rangle \\
& +\left\langle\delta\left(u^{(1)}-v^{(1)}\right) \delta\left(h^{(1)}-g^{(1)}\right) \delta\left(\theta^{(1)}-\varphi^{(1)}\right) \delta\left(c^{(1)}-\psi^{(1)}\right) \delta\left(u^{(2)}-v^{(2)}\right) \delta\left(h^{(2)}-g^{(2)}\right) \delta\left(\theta^{(2)}-\varphi^{(2)}\right)\right. \\
& \left.\delta\left(u^{(3)}-v^{(3)}\right) \delta\left(h^{(3)}-g^{(3)}\right) \delta\left(\theta^{(3)}-\varphi^{(3)}\right) \delta\left(c^{(3)}-\psi^{(3)}\right) \times u_{\beta}^{(2)} \frac{\partial c^{(2)}}{\partial x_{\beta}^{(2)}} \frac{\partial}{\partial \psi^{(2)}} \delta\left(c^{(2)}-\psi^{(2)}\right)\right\rangle \\
& +\left\langle-\delta\left(u^{(1)}-v^{(1)}\right) \delta\left(h^{(1)}-g^{(1)}\right) \delta\left(\theta^{(1)}-\varphi^{(1)}\right) \delta\left(c^{(1)}-\psi^{(1)}\right) \delta\left(u^{(2)}-v^{(2)}\right) \delta\left(h^{(2)}-g^{(2)}\right) \delta\left(\theta^{(2)}-\varphi^{(2)}\right)\right. \\
& \left.\delta\left(u^{(3)}-v^{(3)}\right) \delta\left(h^{(3)}-g^{(3)}\right) \delta\left(\theta^{(3)}-\varphi^{(3)}\right) \delta\left(c^{(3)}-\psi^{(3)}\right) \times D \nabla^{2} c^{(2)} \frac{\partial}{\partial \psi^{(2)}} \delta\left(c^{(2)}-\psi^{(2)}\right)\right\rangle \\
& +\delta\left(u^{(1)}-v^{(1)}\right) \delta\left(h^{(1)}-g^{(1)}\right) \delta\left(\theta^{(1)}-\varphi^{(1)}\right) \delta\left(c^{(1)}-\psi^{(1)}\right) \delta\left(u^{(2)}-v^{(2)}\right) \delta\left(h^{(2)}-g^{(2)}\right) \delta\left(\theta^{(2)}-\varphi^{(2)}\right) \\
& \left.\delta\left(u^{(3)}-v^{(3)}\right) \delta\left(h^{(3)}-g^{(3)}\right) \delta\left(\theta^{(3)}-\varphi^{(3)}\right) \delta\left(c^{(3)}-\psi^{(3)}\right) \times R c^{(2)} \frac{\partial}{\partial \psi^{(2)}} \delta\left(c^{(2)}-\psi^{(2)}\right)\right\rangle \\
& +\left\langle\delta\left(u^{(1)}-v^{(1)}\right) \delta\left(h^{(1)}-g^{(1)}\right) \delta\left(\theta^{(1)}-\varphi^{(1)}\right) \delta\left(c^{(1)}-\psi^{(1)}\right) \delta\left(u^{(2)}-v^{(2)}\right) \delta\left(h^{(2)}-g^{(2)}\right) \delta\left(\theta^{(2)}-\varphi^{(2)}\right)\right. \\
& \left.\delta\left(c^{(2)}-\psi^{(2)}\right) \delta\left(h^{(3)}-g^{(3)}\right) \delta\left(\theta^{(3)}-\varphi^{(3)}\right) \delta\left(c^{(3)}-\psi^{(3)}\right) \times \frac{\partial u_{\alpha}^{(3)} u_{\beta}^{(3)}}{\partial x_{\beta}^{(3)}} \frac{\partial}{\partial v_{\alpha}^{(3)}} \delta\left(u^{(3)}-v^{(3)}\right)\right\rangle \\
& +\left\langle-\delta\left(u^{(1)}-v^{(1)}\right) \delta\left(h^{(1)}-g^{(1)}\right) \delta\left(\theta^{(1)}-\varphi^{(1)}\right) \delta\left(c^{(1)}-\psi^{(1)}\right) \delta\left(u^{(2)}-v^{(2)}\right) \delta\left(h^{(2)}-g^{(2)}\right) \delta\left(\theta^{(2)}-\varphi^{(2)}\right)\right. \\
& \left.\delta\left(c^{(2)}-\psi^{(2)}\right) \delta\left(h^{(3)}-g^{(3)}\right) \delta\left(\theta^{(3)}-\varphi^{(3)}\right) \delta\left(c^{(3)}-\psi^{(3)}\right) \times \frac{\partial h_{\alpha}^{(3)} h_{\beta}^{(3)}}{\partial x_{\beta}^{(3)}} \frac{\partial}{\partial v_{\alpha}^{(3)}} \delta\left(u^{(3)}-v^{(3)}\right)\right\rangle \\
& +\left\langle\delta\left(u^{(1)}-v^{(1)}\right) \delta\left(h^{(1)}-g^{(1)}\right) \delta\left(\theta^{(1)}-\varphi^{(1)}\right) \delta\left(c^{(1)}-\psi^{(1)}\right) \delta\left(u^{(2)}-v^{(2)}\right) \delta\left(h^{(2)}-g^{(2)}\right) \delta\left(\theta^{(2)}-\varphi^{(2)}\right)\right. \\
& \delta\left(c^{(2)}-\psi^{(2)}\right) \delta\left(h^{(3)}-g^{(3)}\right) \delta\left(\theta^{(3)}-\varphi^{(3)}\right) \delta\left(c^{(3)}-\psi^{(3)}\right) \times \frac{1}{4 \pi} \frac{\partial}{\partial x_{\alpha}^{(3)}} \int\left[\frac{\partial u_{\alpha}^{(3)}}{\partial x_{\beta}^{(3)}} \frac{\partial u_{\beta}^{(3)}}{\partial x_{\alpha}^{(3)}}-\frac{\partial h_{\alpha}^{(3)}}{\partial x_{\beta}^{(3)}} \frac{\partial h_{\beta}^{(3)}}{\partial x_{\alpha}^{(3)}}\right] \\
& \left.\times \frac{d \bar{x}^{\prime \prime \prime}}{\left|\bar{x}^{\prime \prime \prime}-\bar{x}^{\prime \prime}\right|} \frac{\partial}{\partial v_{\alpha}^{(3)}} \delta\left(u^{(3)}-v^{(3)}\right)\right\rangle \\
& +\left\langle-\delta\left(u^{(1)}-v^{(1)}\right) \delta\left(h^{(1)}-g^{(1)}\right) \delta\left(\theta^{(1)}-\varphi^{(1)}\right) \delta\left(c^{(1)}-\psi^{(1)}\right) \delta\left(u^{(2)}-v^{(2)}\right) \delta\left(h^{(2)}-g^{(2)}\right) \delta\left(\theta^{(2)}-\varphi^{(2)}\right)\right. \\
& \left.\delta\left(c^{(2)}-\psi^{(2)}\right) \delta\left(h^{(3)}-g^{(3)}\right) \delta\left(\theta^{(3)}-\varphi^{(3)}\right) \delta\left(c^{(3)}-\psi^{(3)}\right) \times V \nabla^{2} u_{\alpha}^{(3)} \frac{\partial}{\partial v_{\alpha}^{(3)}} \delta\left(u^{(3)}-v^{(3)}\right)\right\rangle \\
& +\left\langle-\delta\left(u^{(1)}-v^{(1)}\right) \delta\left(h^{(1)}-g^{(1)}\right) \delta\left(\theta^{(1)}-\varphi^{(1)}\right) \delta\left(c^{(1)}-\psi^{(1)}\right) \delta\left(u^{(2)}-v^{(2)}\right) \delta\left(h^{(2)}-g^{(2)}\right) \delta\left(\theta^{(2)}-\varphi^{(2)}\right)\right. \\
& \left.\delta\left(c^{(2)}-\psi^{(2)}\right) \delta\left(h^{(3)}-g^{(3)}\right) \delta\left(\theta^{(3)}-\varphi^{(3)}\right) \delta\left(c^{(3)}-\psi^{(3)}\right) \times f\left(u_{\alpha}^{(3)}-v_{\alpha}^{(3)}\right) \frac{\partial}{\partial v_{\alpha}^{(3)}} \delta\left(u^{(3)}-v^{(3)}\right)\right\rangle \\
& +\left\langle\delta\left(u^{(1)}-v^{(1)}\right) \delta\left(h^{(1)}-g^{(1)}\right) \delta\left(\theta^{(1)}-\varphi^{(1)}\right) \delta\left(c^{(1)}-\psi^{(1)}\right) \delta\left(u^{(2)}-v^{(2)}\right) \delta\left(h^{(2)}-g^{(2)}\right) \delta\left(\theta^{(2)}-\varphi^{(2)}\right)\right. \\
& \left.\delta\left(c^{(2)}-\psi^{(2)}\right) \delta\left(u^{(3)}-v^{(3)}\right) \delta\left(\theta^{(3)}-\varphi^{(3)}\right) \delta\left(c^{(3)}-\psi^{(3)}\right) \times \frac{\partial h_{\alpha}^{(3)} u_{\beta}^{(3)}}{\partial x_{\beta}^{(3)}} \frac{\partial}{\partial g_{\alpha}^{(3)}} \delta\left(h^{(3)}-g^{(3)}\right)\right\rangle \\
& +\left\langle-\delta\left(u^{(1)}-v^{(1)}\right) \delta\left(h^{(1)}-g^{(1)}\right) \delta\left(\theta^{(1)}-\varphi^{(1)}\right) \delta\left(c^{(1)}-\psi^{(1)}\right) \delta\left(u^{(2)}-v^{(2)}\right) \delta\left(h^{(2)}-g^{(2)}\right) \delta\left(\theta^{(2)}-\varphi^{(2)}\right)\right. \\
& \left.\delta\left(c^{(2)}-\psi^{(2)}\right) \delta\left(u^{(3)}-v^{(3)}\right) \delta\left(\theta^{(3)}-\varphi^{(3)}\right) \delta\left(c^{(3)}-\psi^{(3)}\right) \times \frac{\partial u_{\alpha}^{(3)} h_{\beta}^{(3)}}{\partial x_{\beta}^{(3)}} \frac{\partial}{\partial g_{\alpha}^{(3)}} \delta\left(h^{(3)}-g^{(3)}\right)\right\rangle
\end{aligned}
$$




$$
\begin{aligned}
& +\left\langle-\delta\left(u^{(1)}-v^{(1)}\right) \delta\left(h^{(1)}-g^{(1)}\right) \delta\left(\theta^{(1)}-\varphi^{(1)}\right) \delta\left(c^{(1)}-\psi^{(1)}\right) \delta\left(u^{(2)}-v^{(2)}\right) \delta\left(h^{(2)}-g^{(2)}\right) \delta\left(\theta^{(2)}-\varphi^{(2)}\right)\right. \\
& \left.\delta\left(c^{(2)}-\psi^{(2)}\right) \delta\left(u^{(3)}-v^{(3)}\right) \delta\left(\theta^{(3)}-\varphi^{(3)}\right) \delta\left(c^{(3)}-\psi^{(3)}\right) \times \lambda \nabla^{2} h_{\alpha}^{(3)} \frac{\partial}{\partial g_{\alpha}^{(3)}} \delta\left(h^{(3)}-g^{(3)}\right)\right\rangle \\
& +\left\langle\delta\left(u^{(1)}-v^{(1)}\right) \delta\left(h^{(1)}-g^{(1)}\right) \delta\left(\theta^{(1)}-\varphi^{(1)}\right) \delta\left(c^{(1)}-\psi^{(1)}\right) \delta\left(u^{(2)}-v^{(2)}\right) \delta\left(h^{(2)}-g^{(2)}\right) \delta\left(\theta^{(2)}-\varphi^{(2)}\right)\right. \\
& \left.\delta\left(c^{(2)}-\psi^{(2)}\right) \delta\left(u^{(3)}-v^{(3)}\right) \delta\left(h^{(3)}-g^{(3)}\right) \delta\left(c^{(3)}-\psi^{(3)}\right) \times u_{\beta}^{(3)} \frac{\partial \theta^{(3)}}{\partial x_{\beta}^{(3)}} \frac{\partial}{\partial \varphi^{(3)}} \delta\left(\theta^{(3)}-\varphi^{(3)}\right)\right\rangle \\
& +\left\langle-\delta\left(u^{(1)}-v^{(1)}\right) \delta\left(h^{(1)}-g^{(1)}\right) \delta\left(\theta^{(1)}-\varphi^{(1)}\right) \delta\left(c^{(1)}-\psi^{(1)}\right) \delta\left(u^{(2)}-v^{(2)}\right) \delta\left(h^{(2)}-g^{(2)}\right) \delta\left(\theta^{(2)}-\varphi^{(2)}\right)\right. \\
& \left.\delta\left(c^{(2)}-\psi^{(2)}\right) \delta\left(u^{(3)}-v^{(3)}\right) \delta\left(h^{(3)}-g^{(3)}\right) \delta\left(c^{(3)}-\psi^{(3)}\right) \times \nabla^{2} \theta^{(3)} \frac{\partial}{\partial \varphi^{(3)}} \delta\left(\theta^{(3)}-\varphi^{(3)}\right)\right\rangle \\
& +\left\langle\delta\left(u^{(1)}-v^{(1)}\right) \delta\left(h^{(1)}-g^{(1)}\right) \delta\left(\theta^{(1)}-\varphi^{(1)}\right) \delta\left(c^{(1)}-\psi^{(1)}\right) \delta\left(u^{(2)}-v^{(2)}\right) \delta\left(h^{(2)}-g^{(2)}\right) \delta\left(\theta^{(2)}-\varphi^{(2)}\right)\right. \\
& \left.\delta\left(c^{(2)}-\psi^{(2)}\right) \delta\left(u^{(3)}-v^{(3)}\right) \delta\left(h^{(3)}-g^{(3)}\right) \delta\left(\theta^{(3)}-\varphi^{(3)}\right) \times u_{\beta}^{(3)} \frac{\partial c^{(3)}}{\partial x_{\beta}^{(3)}} \frac{\partial}{\partial \psi^{(3)}} \delta\left(c^{(3)}-\psi^{(3)}\right)\right\rangle \\
& +\left\langle-\delta\left(u^{(1)}-v^{(1)}\right) \delta\left(h^{(1)}-g^{(1)}\right) \delta\left(\theta^{(1)}-\varphi^{(1)}\right) \delta\left(c^{(1)}-\psi^{(1)}\right) \delta\left(u^{(2)}-v^{(2)}\right) \delta\left(h^{(2)}-g^{(2)}\right) \delta\left(\theta^{(2)}-\varphi^{(2)}\right)\right. \\
& \left.\delta\left(u^{(3)}-v^{(3)}\right) \delta\left(h^{(3)}-g^{(3)}\right) \delta\left(\theta^{(3)}-\varphi^{(3)}\right) \times D \nabla^{2} c^{(3)} \frac{\partial}{\partial \psi^{(3)}} \delta\left(c^{(3)}-\psi^{(3)}\right)\right\rangle \\
& +\delta\left(u^{(1)}-v^{(1)}\right) \delta\left(h^{(1)}-g^{(1)}\right) \delta\left(\theta^{(1)}-\varphi^{(1)}\right) \delta\left(c^{(1)}-\psi^{(1)}\right) \delta\left(u^{(2)}-v^{(2)}\right) \delta\left(h^{(2)}-g^{(2)}\right) \delta\left(\theta^{(2)}-\varphi^{(2)}\right) \\
& \left.\delta\left(u^{(3)}-v^{(3)}\right) \delta\left(h^{(3)}-g^{(3)}\right) \delta\left(\theta^{(3)}-\varphi^{(3)}\right) \times R c^{(3)} \frac{\partial}{\partial \psi^{(3)}} \delta\left(c^{(3)}-\psi^{(3)}\right)\right\rangle
\end{aligned}
$$

Various terms in the above equation can be simplified as that they may be expressed in terms of one-, two-, three- and four point distribution functions.

The $1^{\text {st }}$ term in the above equation is simplified as follows

$$
\begin{aligned}
&\left\langle\delta\left(h^{(1)}-g^{(1)}\right) \delta\left(\theta^{(1)}-\varphi^{(1)}\right) \delta\left(c^{(1)}-\psi^{(1)}\right) \delta\left(u^{(2)}-v^{(2)}\right) \delta\left(h^{(2)}-g^{(2)}\right) \delta\left(\theta^{(2)}-\varphi^{(2)}\right) \delta\left(c^{(2)}-\psi^{(2)}\right)\right. \\
&\left.\delta\left(u^{(3)}-v^{(3)}\right) \delta\left(h^{(3)}-g^{(3)}\right) \delta\left(\theta^{(3)}-\varphi^{(3)}\right) \delta\left(c^{(3)}-\psi^{(3)}\right) \times \frac{\partial u_{\alpha}^{(1)} u_{\beta}^{(1)}}{\partial x_{\beta}^{(1)}} \frac{\partial}{\partial v_{\alpha}^{(1)}} \delta\left(u^{(1)}-v^{(1)}\right)\right\rangle \\
&=\left\langle u_{\beta}^{(1)} \delta\left(h^{(1)}-g^{(1)}\right) \delta\left(\theta^{(1)}-\varphi^{(1)}\right) \delta\left(c^{(1)}-\psi^{(1)}\right) \delta\left(u^{(2)}-v^{(2)}\right) \delta\left(h^{(2)}-g^{(2)}\right) \delta\left(\theta^{(2)}-\varphi^{(2)}\right) \delta\left(c^{(2)}-\psi^{(2)}\right)\right. \\
&\left.\delta\left(u^{(3)}-v^{(3)}\right) \delta\left(h^{(3)}-g^{(3)}\right) \delta\left(\theta^{(3)}-\varphi^{(3)}\right) \delta\left(c^{(3)}-\psi^{(3)}\right) \times \frac{\partial u_{\alpha}^{(1)}}{\partial x_{\beta}^{(1)}} \frac{\partial}{\partial v_{\alpha}^{(1)}} \delta\left(u^{(1)}-v^{(1)}\right)\right\rangle \\
&=\left\langle-u_{\beta}^{(1)} \delta\left(h^{(1)}-g^{(1)}\right) \delta\left(\theta^{(1)}-\varphi^{(1)}\right) \delta\left(c^{(1)}-\psi^{(1)}\right) \delta\left(u^{(2)}-v^{(2)}\right) \delta\left(h^{(2)}-g^{(2)}\right) \delta\left(\theta^{(2)}-\varphi^{(2)}\right) \delta\left(c^{(2)}-\psi^{(2)}\right)\right. \\
&\left.\delta\left(u^{(3)}-v^{(3)}\right) \delta\left(h^{(3)}-g^{(3)}\right) \delta\left(\theta^{(3)}-\varphi^{(3)}\right) \delta\left(c^{(3)}-\psi^{(3)}\right) \times \frac{\partial u_{\alpha}^{(1)}}{\partial v_{\alpha}^{(1)}} \frac{\partial}{\partial x_{\beta}^{(1)}} \delta\left(u^{(1)}-v^{(1)}\right)\right\rangle ;\left(\text { since } \frac{\partial u_{\alpha}^{(1)}}{\partial v_{\alpha}^{(1)}}=1\right) \\
&=\left\langle-\delta\left(h^{(1)}-g^{(1)}\right) \delta\left(\theta^{(1)}-\varphi^{(1)}\right) \delta\left(c^{(1)}-\psi^{(1)}\right) \delta\left(u^{(2)}-v^{(2)}\right) \delta\left(h^{(2)}-g^{(2)}\right) \delta\left(\theta^{(2)}-\varphi^{(2)}\right) \delta\left(c^{(2)}-\psi^{(2)}\right)\right. \\
&\left.\delta\left(u^{(3)}-v^{(3)}\right) \delta\left(h^{(3)}-g^{(3)}\right) \delta\left(\theta^{(3)}-\varphi^{(3)}\right) \delta\left(c^{(3)}-\psi^{(3)}\right) \times u_{\beta}^{(1)} \frac{\partial}{\partial x_{\beta}^{(1)}} \delta\left(u^{(1)}-v^{(1)}\right)\right\rangle
\end{aligned}
$$

Similarly, sixth, ninth and eleventh terms of right hand-side of equation (70) can be simplified as follows;

$$
\begin{aligned}
& \left\langle\delta\left(u^{(1)}-v^{(1)}\right) \delta\left(\theta^{(1)}-\varphi^{(1)}\right) \delta\left(c^{(1)}-\psi^{(1)}\right) \delta\left(u^{(2)}-v^{(2)}\right) \delta\left(h^{(2)}-g^{(2)}\right) \delta\left(\theta^{(2)}-\varphi^{(2)}\right) \delta\left(c^{(2)}-\psi^{(2)}\right)\right. \\
& \left.\delta\left(u^{(3)}-v^{(3)}\right) \delta\left(h^{(3)}-g^{(3)}\right) \delta\left(\theta^{(3)}-\varphi^{(3)}\right) \delta\left(c^{(3)}-\psi^{(3)}\right) \times \frac{\partial h_{\alpha}^{(1)} u_{\beta}^{(1)}}{\partial x_{\beta}^{(1)}} \frac{\partial}{\partial g_{\alpha}^{(1)}} \delta\left(h^{(1)}-g^{(1)}\right)\right\rangle
\end{aligned}
$$

nineth term, 


$$
\begin{aligned}
& \left\langle\delta\left(u^{(1)}-v^{(1)}\right) \delta\left(h^{(1)}-g^{(1)}\right) \delta\left(c^{(1)}-\psi^{(1)}\right) \delta\left(u^{(2)}-v^{(2)}\right) \delta\left(h^{(2)}-g^{(2)}\right) \delta\left(\theta^{(2)}-\varphi^{(2)}\right) \delta\left(c^{(2)}-\psi^{(2)}\right)\right. \\
& \left.\delta\left(u^{(3)}-v^{(3)}\right) \delta\left(h^{(3)}-g^{(3)}\right) \delta\left(\theta^{(3)}-\varphi^{(3)}\right) \delta\left(c^{(3)}-\psi^{(3)}\right) \times u_{\beta}^{(1)} \frac{\partial \theta^{(1)}}{\partial x_{\beta}^{(1)}} \frac{\partial}{\partial \varphi^{(1)}} \delta\left(\theta^{(1)}-\varphi^{(1)}\right)\right\rangle \\
= & \left\langle-\delta\left(u^{(1)}-v^{(1)}\right) \delta\left(h^{(1)}-g^{(1)}\right) \delta\left(c^{(1)}-\psi^{(1)}\right) \delta\left(u^{(2)}-v^{(2)}\right) \delta\left(h^{(2)}-g^{(2)}\right) \delta\left(\theta^{(2)}-\varphi^{(2)}\right) \delta\left(c^{(2)}-\psi^{(2)}\right)\right. \\
& \left.\delta\left(u^{(3)}-v^{(3)}\right) \delta\left(h^{(3)}-g^{(3)}\right) \delta\left(\theta^{(3)}-\varphi^{(3)}\right) \delta\left(c^{(3)}-\psi^{(3)}\right) \times u_{\beta}^{(1)} \frac{\partial}{\partial x_{\beta}^{(1)}} \delta\left(\theta^{(1)}-\varphi^{(1)}\right)\right\rangle
\end{aligned}
$$

and eleventh term

$$
\begin{aligned}
& \left\langle\delta\left(u^{(1)}-v^{(1)}\right) \delta\left(h^{(1)}-g^{(1)}\right) \delta\left(\theta^{(1)}-\varphi^{(1)}\right) \delta\left(u^{(2)}-v^{(2)}\right) \delta\left(h^{(2)}-g^{(2)}\right) \delta\left(\theta^{(2)}-\varphi^{(2)}\right) \delta\left(c^{(2)}-\psi^{(2)}\right)\right. \\
& \left.\delta\left(u^{(3)}-v^{(3)}\right) \delta\left(h^{(3)}-g^{(3)}\right) \delta\left(\theta^{(3)}-\varphi^{(3)}\right) \delta\left(c^{(3)}-\psi^{(3)}\right) \times u_{\beta}^{(1)} \frac{\partial c^{(1)}}{\partial x_{\beta}^{(1)}} \frac{\partial}{\partial \psi^{(1)}} \delta\left(c^{(1)}-\psi^{(1)}\right)\right\rangle \\
& =\left\langle-\delta\left(u^{(1)}-v^{(1)}\right) \delta\left(h^{(1)}-g^{(1)}\right) \delta\left(\theta^{(1)}-\varphi^{(1)}\right) \delta\left(u^{(2)}-v^{(2)}\right) \delta\left(h^{(2)}-g^{(2)}\right) \delta\left(\theta^{(2)}-\varphi^{(2)}\right) \delta\left(c^{(2)}-\psi^{(2)}\right)\right. \\
& \left.\delta\left(u^{(3)}-v^{(3)}\right) \delta\left(h^{(3)}-g^{(3)}\right) \delta\left(\theta^{(3)}-\varphi^{(3)}\right) \delta\left(c^{(3)}-\psi^{(3)}\right) \times u_{\beta}^{(1)} \frac{\partial}{\partial x_{\beta}^{(1)}} \delta\left(c^{(1)}-\psi^{(1)}\right)\right\rangle
\end{aligned}
$$

Adding these equations from (71) to (74), we get

$$
\begin{aligned}
& \left\langle-\delta\left(h^{(1)}-g^{(1)}\right) \delta\left(\theta^{(1)}-\varphi^{(1)}\right) \delta\left(c^{(1)}-\psi^{(1)}\right) \delta\left(u^{(2)}-v^{(2)}\right) \delta\left(h^{(2)}-g^{(2)}\right) \delta\left(\theta^{(2)}-\varphi^{(2)}\right) \delta\left(c^{(2)}-\psi^{(2)}\right)\right. \\
& \left.\delta\left(u^{(3)}-v^{(3)}\right) \delta\left(h^{(3)}-g^{(3)}\right) \delta\left(\theta^{(3)}-\varphi^{(3)}\right) \delta\left(c^{(3)}-\psi^{(3)}\right) \times u_{\beta}^{(1)} \frac{\partial}{\partial x_{\beta}^{(1)}} \delta\left(u^{(1)}-v^{(1)}\right)\right\rangle \\
& +\left\langle-\delta\left(u^{(1)}-v^{(1)}\right) \delta\left(\theta^{(1)}-\varphi^{(1)}\right) \delta\left(c^{(1)}-\psi^{(1)}\right) \delta\left(u^{(2)}-v^{(2)}\right) \delta\left(h^{(2)}-g^{(2)}\right) \delta\left(\theta^{(2)}-\varphi^{(2)}\right) \delta\left(c^{(2)}-\psi^{(2)}\right)\right. \\
& \left.\delta\left(u^{(3)}-v^{(3)}\right) \delta\left(h^{(3)}-g^{(3)}\right) \delta\left(\theta^{(3)}-\varphi^{(3)}\right) \delta\left(c^{(3)}-\psi^{(3)}\right) \times u_{\beta}^{(1)} \frac{\partial}{\partial x_{\beta}^{(1)}} \delta\left(h^{(1)}-g^{(1)}\right)\right\rangle \\
& +\left\langle-\delta\left(u^{(1)}-v^{(1)}\right) \delta\left(h^{(1)}-g^{(1)}\right) \delta\left(c^{(1)}-\psi^{(1)}\right) \delta\left(u^{(2)}-v^{(2)}\right) \delta\left(h^{(2)}-g^{(2)}\right) \delta\left(\theta^{(2)}-\varphi^{(2)}\right) \delta\left(c^{(2)}-\psi^{(2)}\right)\right. \\
& \left.\delta\left(u^{(3)}-v^{(3)}\right) \delta\left(h^{(3)}-g^{(3)}\right) \delta\left(\theta^{(3)}-\varphi^{(3)}\right) \delta\left(c^{(3)}-\psi^{(3)}\right) \times u_{\beta}^{(1)} \frac{\partial}{\partial x_{\beta}^{(1)}} \delta\left(\theta^{(1)}-\varphi^{(1)}\right)\right\rangle \\
& +\left\langle-\delta\left(u^{(1)}-v^{(1)}\right) \delta\left(h^{(1)}-g^{(1)}\right) \delta\left(\theta^{(1)}-\varphi^{(1)}\right) \delta\left(u^{(2)}-v^{(2)}\right) \delta\left(h^{(2)}-g^{(2)}\right) \delta\left(\theta^{(2)}-\varphi^{(2)}\right) \delta\left(c^{(2)}-\psi^{(2)}\right)\right. \\
& \left.\delta\left(u^{(3)}-v^{(3)}\right) \delta\left(h^{(3)}-g^{(3)}\right) \delta\left(\theta^{(3)}-\varphi^{(3)}\right) \delta\left(c^{(3)}-\psi^{(3)}\right) \times u_{\beta}^{(1)} \frac{\partial}{\partial x_{\beta}^{(1)}} \delta\left(c^{(1)}-\psi^{(1)}\right)\right\rangle \\
& =-\frac{\partial}{\partial x_{\beta}^{(1)}}\left\langleu _ { \beta } ^ { ( 1 ) } \left\langle-\delta\left(u^{(1)}-v^{(1)}\right) \delta\left(h^{(1)}-g^{(1)}\right) \delta\left(\theta^{(1)}-\varphi^{(1)}\right) \delta\left(c^{(1)}-\psi^{(1)}\right) \delta\left(u^{(2)}-v^{(2)}\right) \delta\left(h^{(2)}-g^{(2)}\right)\right.\right. \\
& \left.\delta\left(\theta^{(2)}-\varphi^{(2)}\right) \delta\left(c^{(2)}-\psi^{(2)}\right) \delta\left(u^{(3)}-v^{(3)}\right) \delta\left(h^{(3)}-g^{(3)}\right) \delta\left(\theta^{(3)}-\varphi^{(3)}\right) \delta\left(c^{(3)}-\psi^{(3)}\right)\right\rangle \\
& =-\frac{\partial}{\partial x_{\beta}^{(1)}} v_{\beta}^{(1)} F_{3}^{(1,2,3)}[\text { Applying the properties of distribution functions] }
\end{aligned}
$$

Similarly, $14^{\text {th }}, 19^{\text {th }}, 22^{\text {nd }}$ and $24^{\text {th }}$ terms of right hand-side of equation (17) can be simplified as follows;

$$
\begin{aligned}
& \left\langle\delta\left(u^{(1)}-v^{(1)}\right) \delta\left(h^{(1)}-g^{(1)}\right) \delta\left(\theta^{(1)}-\varphi^{(1)}\right) \delta\left(c^{(1)}-\psi^{(1)}\right) \delta\left(h^{(2)}-g^{(2)}\right) \delta\left(\theta^{(2)}-\varphi^{(2)}\right) \delta\left(c^{(2)}-\psi^{(2)}\right)\right. \\
& \left.\delta\left(u^{(3)}-v^{(3)}\right) \delta\left(h^{(3)}-g^{(3)}\right) \delta\left(\theta^{(3)}-\varphi^{(3)}\right) \delta\left(c^{(3)}-\psi^{(3)}\right) \times \frac{\partial u_{\alpha}^{(2)} u_{\beta}^{(2)}}{\partial x_{\beta}^{(2)}} \frac{\partial}{\partial v_{\alpha}^{(2)}} \delta\left(u^{(2)}-v^{(2)}\right)\right\rangle
\end{aligned}
$$




$$
\begin{aligned}
& =\left\langle-\delta\left(u^{(1)}-v^{(1)}\right) \delta\left(h^{(1)}-g^{(1)}\right) \delta\left(\theta^{(1)}-\varphi^{(1)}\right) \delta\left(c^{(1)}-\psi^{(1)}\right) \delta\left(h^{(2)}-g^{(2)}\right) \delta\left(\theta^{(2)}-\varphi^{(2)}\right)\right. \\
& \left.\delta\left(c^{(2)}-\psi^{(2)}\right) \delta\left(u^{(3)}-v^{(3)}\right) \delta\left(h^{(3)}-g^{(3)}\right) \delta\left(\theta^{(3)}-\varphi^{(3)}\right) \delta\left(c^{(3)}-\psi^{(3)}\right) \times u_{\beta}^{(2)} \frac{\partial}{\partial x_{\beta}^{(2)}} \delta\left(u^{(2)}-v^{(2)}\right)\right\rangle
\end{aligned}
$$

$19^{\text {th }}$ term,

$$
\begin{aligned}
& \left\langle\delta\left(u^{(1)}-v^{(1)}\right) \delta\left(h^{(1)}-g^{(1)}\right) \delta\left(\theta^{(1)}-\varphi^{(1)}\right) \delta\left(c^{(1)}-\psi^{(1)}\right) \delta\left(u^{(2)}-v^{(2)}\right) \delta\left(\theta^{(2)}-\varphi^{(2)}\right) \delta\left(c^{(2)}-\psi^{(2)}\right)\right. \\
& \left.\delta\left(u^{(3)}-v^{(3)}\right) \delta\left(h^{(3)}-g^{(3)}\right) \delta\left(\theta^{(3)}-\varphi^{(3)}\right) \delta\left(c^{(3)}-\psi^{(3)}\right) \times \frac{\partial h_{\alpha}^{(2)} u_{\beta}^{(2)}}{\partial x_{\beta}^{(2)}} \frac{\partial}{\partial g_{\alpha}^{(2)}} \delta\left(h^{(2)}-g^{(2)}\right)\right\rangle \\
& =\left\langle-\delta\left(u^{(1)}-v^{(1)}\right) \delta\left(h^{(1)}-g^{(1)}\right) \delta\left(\theta^{(1)}-\varphi^{(1)}\right) \delta\left(c^{(1)}-\psi^{(1)}\right) \delta\left(u^{(2)}-v^{(2)}\right) \delta\left(\theta^{(2)}-\varphi^{(2)}\right) \delta\left(c^{(2)}-\psi^{(2)}\right)\right. \\
& \left.\delta\left(u^{(3)}-v^{(3)}\right) \delta\left(h^{(3)}-g^{(3)}\right) \delta\left(\theta^{(3)}-\varphi^{(3)}\right) \delta\left(c^{(3)}-\psi^{(3)}\right) \times u_{\beta}^{(2)} \frac{\partial}{\partial x_{\beta}^{(2)}} \delta\left(h^{(2)}-g^{(2)}\right)\right\rangle
\end{aligned}
$$

$22^{\text {nd }}$ term,

$$
\begin{aligned}
& \left\langle\delta\left(u^{(1)}-v^{(1)}\right) \delta\left(h^{(1)}-g^{(1)}\right) \delta\left(\theta^{(1)}-\varphi^{(1)}\right) \delta\left(c^{(1)}-\psi^{(1)}\right) \delta\left(u^{(2)}-v^{(2)}\right) \delta\left(h^{(2)}-g^{(2)}\right) \delta\left(c^{(2)}-\psi^{(2)}\right)\right. \\
& \left.\delta\left(u^{(3)}-v^{(3)}\right) \delta\left(h^{(3)}-g^{(3)}\right) \delta\left(\theta^{(3)}-\varphi^{(3)}\right) \delta\left(c^{(3)}-\psi^{(3)}\right) \times u_{\beta}^{(2)} \frac{\partial \theta^{(2)}}{\partial x_{\beta}^{(2)}} \frac{\partial}{\partial \varphi^{(2)}} \delta\left(\theta^{(2)}-\varphi^{(2)}\right)\right\rangle \\
& =\left\langle-\delta\left(u^{(1)}-v^{(1)}\right) \delta\left(h^{(1)}-g^{(1)}\right) \delta\left(\theta^{(1)}-\varphi^{(1)}\right) \delta\left(c^{(1)}-\psi^{(1)}\right) \delta\left(u^{(2)}-v^{(2)}\right) \delta\left(h^{(2)}-g^{(2)}\right) \delta\left(c^{(2)}-\psi^{(2)}\right)\right. \\
& \left.\delta\left(u^{(3)}-v^{(3)}\right) \delta\left(h^{(3)}-g^{(3)}\right) \delta\left(\theta^{(3)}-\varphi^{(3)}\right) \delta\left(c^{(3)}-\psi^{(3)}\right) \times u_{\beta}^{(2)} \frac{\partial}{\partial x_{\beta}^{(2)}} \delta\left(\theta^{(2)}-\varphi^{(2)}\right)\right\rangle
\end{aligned}
$$

And $24^{\text {th }}$ term,

$$
\begin{aligned}
& \left\langle\delta\left(u^{(1)}-v^{(1)}\right) \delta\left(h^{(1)}-g^{(1)}\right) \delta\left(\theta^{(1)}-\varphi^{(1)}\right) \delta\left(c^{(1)}-\psi^{(1)}\right) \delta\left(u^{(2)}-v^{(2)}\right) \delta\left(h^{(2)}-g^{(2)}\right) \delta\left(\theta^{(2)}-\varphi^{(2)}\right)\right. \\
& \left.\delta\left(u^{(3)}-v^{(3)}\right) \delta\left(h^{(3)}-g^{(3)}\right) \delta\left(\theta^{(3)}-\varphi^{(3)}\right) \delta\left(c^{(3)}-\psi^{(3)}\right) \times u_{\beta}^{(2)} \frac{\partial c^{(2)}}{\partial x_{\beta}^{(2)}} \frac{\partial}{\partial \psi^{(2)}} \delta\left(c^{(2)}-\psi^{(2)}\right)\right\rangle \\
& =\left\langle-\delta\left(u^{(1)}-v^{(1)}\right) \delta\left(h^{(1)}-g^{(1)}\right) \delta\left(\theta^{(1)}-\varphi^{(1)}\right) \delta\left(c^{(1)}-\psi^{(1)}\right) \delta\left(u^{(2)}-v^{(2)}\right) \delta\left(h^{(2)}-g^{(2)}\right) \delta\left(\theta^{(2)}-\varphi^{(2)}\right)\right. \\
& \left.\delta\left(u^{(3)}-v^{(3)}\right) \delta\left(h^{(3)}-g^{(3)}\right) \delta\left(\theta^{(3)}-\varphi^{(3)}\right) \delta\left(c^{(3)}-\psi^{(3)}\right) \times u_{\beta}^{(2)} \frac{\partial}{\partial x_{\beta}^{(2)}} \delta\left(c^{(2)}-\psi^{(2)}\right)\right\rangle
\end{aligned}
$$

Adding equations (76) to (79), we get

$$
\begin{aligned}
& -\frac{\partial}{\partial x_{\beta}^{(2)}}\left\langleu _ { \beta } ^ { ( 2 ) } \left\langle\delta\left(u^{(1)}-v^{(1)}\right) \delta\left(h^{(1)}-g^{(1)}\right) \delta\left(\theta^{(1)}-\varphi^{(1)}\right) \delta\left(c^{(1)}-\psi^{(1)}\right) \delta\left(u^{(2)}-v^{(2)}\right) \delta\left(h^{(2)}-g^{(2)}\right)=-v_{\beta}^{(2)} \frac{\partial F_{3}^{(1,2,3)}}{\partial x_{\beta}^{(2)}}\right.\right. \\
& \left.\delta\left(\theta^{(2)}-\varphi^{(2)}\right) \delta\left(c^{(2)}-\psi^{(2)}\right) \delta\left(u^{(3)}-v^{(3)}\right) \delta\left(h^{(3)}-g^{(3)}\right) \delta\left(\theta^{(3)}-\varphi^{(3)}\right) \delta\left(c^{(3)}-\psi^{(3)}\right)\right\rangle
\end{aligned}
$$

Similarly, $27^{\text {th }}, 32^{\text {nd }}, 35^{\text {th }}$ and $37^{\text {th }}$ terms of right hand-side of equation (70) can be simplified as follows;

$$
\begin{gathered}
\left\langle\delta\left(u^{(1)}-v^{(1)}\right) \delta\left(h^{(1)}-g^{(1)}\right) \delta\left(\theta^{(1)}-\varphi^{(1)}\right) \delta\left(c^{(1)}-\psi^{(1)}\right) \delta\left(u^{(2)}-v^{(2)}\right) \delta\left(h^{(2)}-g^{(2)}\right) \delta\left(\theta^{(2)}-\varphi^{(2)}\right)\right. \\
\left.\delta\left(c^{(2)}-\psi^{(2)}\right) \delta\left(h^{(3)}-g^{(3)}\right) \delta\left(\theta^{(3)}-\varphi^{(3)}\right) \delta\left(c^{(3)}-\psi^{(3)}\right) \times \frac{\partial u_{\alpha}^{(3)} u_{\beta}^{(3)}}{\partial x_{\beta}^{(3)}} \frac{\partial}{\partial v_{\alpha}^{(2)}} \delta\left(u^{(3)}-v^{(3)}\right)\right\rangle \\
=\left\langle-\delta\left(u^{(1)}-v^{(1)}\right) \delta\left(h^{(1)}-g^{(1)}\right) \delta\left(\theta^{(1)}-\varphi^{(1)}\right) \delta\left(c^{(1)}-\psi^{(1)}\right) \delta\left(u^{(3)}-v^{(3)}\right) \delta\left(h^{(2)}-g^{(2)}\right)\right.
\end{gathered}
$$




$$
\left.\delta\left(\theta^{(2)}-\varphi^{(2)}\right) \delta\left(c^{(2)}-\psi^{(2)}\right) \delta\left(h^{(3)}-g^{(3)}\right) \delta\left(\theta^{(3)}-\varphi^{(3)}\right) \delta\left(c^{(3)}-\psi^{(3)}\right) \times u_{\beta}^{(3)} \frac{\partial}{\partial x_{\beta}^{(3)}} \delta\left(u^{(3)}-v^{(3)}\right)\right\rangle
$$

$32^{\text {nd }}$ term,

$$
\begin{aligned}
& \left\langle\delta\left(u^{(1)}-v^{(1)}\right) \delta\left(h^{(1)}-g^{(1)}\right) \delta\left(\theta^{(1)}-\varphi^{(1)}\right) \delta\left(c^{(1)}-\psi^{(1)}\right) \delta\left(u^{(2)}-v^{(2)}\right) \delta\left(h^{(2)}-g^{(2)}\right) \delta\left(\theta^{(2)}-\varphi^{(2)}\right)\right. \\
& \left.\delta\left(c^{(2)}-\psi^{(2)}\right) \delta\left(u^{(3)}-v^{(3)}\right) \delta\left(\theta^{(3)}-\varphi^{(3)}\right) \delta\left(c^{(3)}-\psi^{(3)}\right) \times \frac{\partial h_{\alpha}^{(3)} u_{\beta}^{(3)}}{\partial x_{\beta}^{(3)}} \frac{\partial}{\partial g_{\alpha}^{(3)}} \delta\left(h^{(3)}-g^{(3)}\right)\right\rangle \\
& =\left\langle-\delta\left(u^{(1)}-v^{(1)}\right) \delta\left(h^{(1)}-g^{(1)}\right) \delta\left(\theta^{(1)}-\varphi^{(1)}\right) \delta\left(c^{(1)}-\psi^{(1)}\right) \delta\left(u^{(2)}-v^{(2)}\right) \delta\left(h^{(2)}-g^{(2)}\right) \delta\left(\theta^{(2)}-\varphi^{(2)}\right)\right. \\
& \left.\delta\left(c^{(2)}-\psi^{(2)}\right) \delta\left(u^{(3)}-v^{(3)}\right) \delta\left(\theta^{(3)}-\varphi^{(3)}\right) \delta\left(c^{(3)}-\psi^{(3)}\right) \times u_{\beta}^{(3)} \frac{\partial}{\partial x_{\beta}^{(3)}} \delta\left(h^{(3)}-g^{(3)}\right)\right\rangle
\end{aligned}
$$

$35^{\text {th }}$ term,

$$
\begin{aligned}
& \left\langle\delta\left(u^{(1)}-v^{(1)}\right) \delta\left(h^{(1)}-g^{(1)}\right) \delta\left(\theta^{(1)}-\varphi^{(1)}\right) \delta\left(c^{(1)}-\psi^{(1)}\right) \delta\left(u^{(2)}-v^{(2)}\right) \delta\left(h^{(2)}-g^{(2)}\right) \delta\left(\theta^{(2)}-\varphi^{(2)}\right)\right. \\
& \left.\delta\left(c^{(2)}-\psi^{(2)}\right) \delta\left(u^{(3)}-v^{(3)}\right) \delta\left(h^{(3)}-g^{(3)}\right) \delta\left(c^{(3)}-\psi^{(3)}\right) \times u_{\beta}^{(3)} \frac{\partial \theta^{(3)}}{\partial x_{\beta}^{(3)}} \frac{\partial}{\partial \varphi^{(3)}} \delta\left(\theta^{(3)}-\varphi^{(3)}\right)\right\rangle \\
& =\left\langle-\delta\left(u^{(1)}-v^{(1)}\right) \delta\left(h^{(1)}-g^{(1)}\right) \delta\left(\theta^{(1)}-\varphi^{(1)}\right) \delta\left(c^{(1)}-\psi^{(1)}\right) \delta\left(u^{(2)}-v^{(2)}\right) \delta\left(h^{(2)}-g^{(2)}\right) \delta\left(\theta^{(2)}-\varphi^{(2)}\right)\right. \\
& \left.\delta\left(c^{(2)}-\psi^{(2)}\right) \delta\left(u^{(3)}-v^{(3)}\right) \delta\left(h^{(3)}-g^{(3)}\right) \delta\left(c^{(3)}-\psi^{(3)}\right) \times u_{\beta}^{(3)} \frac{\partial}{\partial x_{\beta}^{(3)}} \delta\left(\theta^{(3)}-\varphi^{(3)}\right)\right\rangle
\end{aligned}
$$

and $37^{\text {th }}$ term,

$$
\begin{aligned}
& \left\langle\delta\left(u^{(1)}-v^{(1)}\right) \delta\left(h^{(1)}-g^{(1)}\right) \delta\left(\theta^{(1)}-\varphi^{(1)}\right) \delta\left(c^{(1)}-\psi^{(1)}\right) \delta\left(u^{(2)}-v^{(2)}\right) \delta\left(h^{(2)}-g^{(2)}\right) \delta\left(\theta^{(2)}-\varphi^{(2)}\right)\right. \\
& \left.\delta\left(c^{(2)}-\psi^{(2)}\right) \delta\left(u^{(3)}-v^{(3)}\right) \delta\left(h^{(3)}-g^{(3)}\right) \delta\left(\theta^{(3)}-\varphi^{(3)}\right) \times u_{\beta}^{(3)} \frac{\partial c^{(3)}}{\partial x_{\beta}^{(3)}} \frac{\partial}{\partial \psi^{(3)}} \delta\left(c^{(3)}-\psi^{(3)}\right)\right\rangle \\
& =\left\langle-\delta\left(u^{(1)}-v^{(1)}\right) \delta\left(h^{(1)}-g^{(1)}\right) \delta\left(\theta^{(1)}-\varphi^{(1)}\right) \delta\left(c^{(1)}-\psi^{(1)}\right) \delta\left(u^{(2)}-v^{(2)}\right) \delta\left(h^{(2)}-g^{(2)}\right) \delta\left(\theta^{(2)}-\varphi^{(2)}\right)\right. \\
& \left.\delta\left(c^{(2)}-\psi^{(2)}\right) \delta\left(u^{(3)}-v^{(3)}\right) \delta\left(h^{(3)}-g^{(3)}\right) \delta\left(\theta^{(3)}-\varphi^{(3)}\right) \times u_{\beta}^{(3)} \frac{\partial}{\partial x_{\beta}^{(3)}} \delta\left(c^{(3)}-\psi^{(3)}\right)\right\rangle
\end{aligned}
$$

Adding equations (81) to (84), we get

$$
\begin{aligned}
& -\frac{\partial}{\partial x_{\beta}^{(3)}}\left\langleu _ { \beta } ^ { ( 3 ) } \left\langle-\delta\left(u^{(1)}-v^{(1)}\right) \delta\left(h^{(1)}-g^{(1)}\right) \delta\left(\theta^{(1)}-\varphi^{(1)}\right) \delta\left(c^{(1)}-\psi^{(1)}\right) \delta\left(u^{(2)}-v^{(2)}\right) \delta\left(h^{(2)}-g^{(2)}\right)=-v_{\beta}^{(3)} \frac{\partial F_{3}^{(1,2,3)}}{\partial x_{\beta}^{(3)}}\right.\right. \\
& \left.\delta\left(\theta^{(2)}-\varphi^{(2)}\right) \delta\left(c^{(2)}-\psi^{(2)}\right) \delta\left(u^{(3)}-v^{(3)}\right) \delta\left(h^{(3)}-g^{(3)}\right) \delta\left(\theta^{(3)}-\varphi^{(3)}\right) \delta\left(c^{(3)}-\psi^{(3)}\right)\right\rangle
\end{aligned}
$$

Similarly, $2^{\text {nd }}, 7^{\text {th }}, 15^{\text {th }}, 20^{\text {th }}, 28^{\text {th }}$ and $33^{\text {rd }}$ terms of right hand-side of equation (70) can be simplified as follows;

$$
\begin{aligned}
& \left\langle-\delta\left(h^{(1)}-g^{(1)}\right) \delta\left(\theta^{(1)}-\varphi^{(1)}\right) \delta\left(c^{(1)}-\psi^{(1)}\right) \delta\left(u^{(2)}-v^{(2)}\right) \delta\left(h^{(2)}-g^{(2)}\right) \delta\left(\theta^{(2)}-\varphi^{(2)}\right) \delta\left(c^{(2)}-\psi^{(2)}\right)\right. \\
& \left.\delta\left(u^{(3)}-v^{(3)}\right) \delta\left(h^{(3)}-g^{(3)}\right) \delta\left(\theta^{(3)}-\varphi^{(3)}\right) \delta\left(c^{(3)}-\psi^{(3)}\right) \times \frac{\partial h_{\alpha}^{(1)} h_{\beta}^{(1)}}{\partial x_{\beta}^{(1)}} \frac{\partial}{\partial v_{\alpha}^{(1)}} \delta\left(u^{(1)}-v^{(1)}\right)\right\rangle
\end{aligned}=-\frac{\partial g_{\alpha}^{(1)}}{\partial v_{\alpha}^{(1)}} \frac{\partial F_{3}^{(1,2,3)}}{\partial x_{\beta}^{(1)}}
$$

$7^{\text {th }}$ term,

$$
\begin{aligned}
& \left\langle-\delta\left(u^{(1)}-v^{(1)}\right) \delta\left(\theta^{(1)}-\varphi^{(1)}\right) \delta\left(c^{(1)}-\psi^{(1)}\right) \delta\left(u^{(2)}-v^{(2)}\right) \delta\left(h^{(2)}-g^{(2)}\right) \delta\left(\theta^{(2)}-\varphi^{(2)}\right) \delta\left(c^{(2)}-\psi^{(2)}\right)\right. \\
& \left.\delta\left(u^{(3)}-v^{(3)}\right) \delta\left(h^{(3)}-g^{(3)}\right) \delta\left(\theta^{(3)}-\varphi^{(3)}\right) \delta\left(c^{(3)}-\psi^{(3)}\right) \times \frac{\partial u_{\alpha}^{(1)} h_{\beta}^{(1)}}{\partial x_{\beta}^{(1)}} \frac{\partial}{\partial g_{\alpha}} \delta\left(h^{(1)}-g^{(1)}\right)\right\rangle
\end{aligned}=-g_{\beta}^{(1)} \frac{\partial v_{\alpha}^{(1)}}{\partial g_{\alpha}^{(1)}} \frac{\partial F_{3}^{(1,2,3)}}{\partial x_{\beta}^{(1)}}
$$


$15^{\text {th }}$ term,

$$
\begin{aligned}
& \left\langle-\delta\left(u^{(1)}-v^{(1)}\right) \delta\left(h^{(1)}-g^{(1)}\right) \delta\left(\theta^{(1)}-\varphi^{(1)}\right) \delta\left(c^{(1)}-\psi^{(1)}\right) \delta\left(h^{(2)}-g^{(2)}\right) \delta\left(\theta^{(2)}-\varphi^{(2)}\right) \delta\left(c^{(2)}-\psi^{(2)}\right)\right. \\
& \left.\delta\left(u^{(3)}-v^{(3)}\right) \delta\left(h^{(3)}-g^{(3)}\right) \delta\left(\theta^{(3)}-\varphi^{(3)}\right) \delta\left(c^{(3)}-\psi^{(3)}\right) \times \frac{\partial h_{\alpha}^{(2)} h_{\beta}^{(2)}}{\partial x_{\beta}^{(2)}} \frac{\partial}{\partial v_{\alpha}^{(2)}} \delta\left(u^{(2)}-v^{(2)}\right)\right\rangle \frac{\partial g_{\alpha}^{(2)}}{\partial v_{\alpha}^{(2)}} \frac{\partial F_{3}^{(1,2,3)}}{\partial x_{\beta}^{(2)}}
\end{aligned}
$$

$20^{\text {th }}$ term,

$$
\begin{aligned}
& \left\langle-\delta\left(u^{(1)}-v^{(1)}\right) \delta\left(h^{(1)}-g^{(1)}\right) \delta\left(\theta^{(1)}-\varphi^{(1)}\right) \delta\left(c^{(1)}-\psi^{(1)}\right) \delta\left(u^{(2)}-v^{(2)}\right) \delta\left(\theta^{(2)}-\varphi^{(2)}\right) \delta\left(c^{(2)}-\psi^{(2)}\right)\right. \\
& \left.\delta\left(u^{(3)}-v^{(3)}\right) \delta\left(h^{(3)}-g^{(3)}\right) \delta\left(\theta^{(3)}-\varphi^{(3)}\right) \delta\left(c^{(3)}-\psi^{(3)}\right) \times \frac{\partial u_{\alpha}^{(2)} h_{\beta}^{(2)}}{\partial x_{\beta}^{(2)}} \frac{\partial}{\partial g_{\alpha}^{(2)}} \delta\left(h^{(2)}-g^{(2)}\right)\right\rangle
\end{aligned}=-g_{\beta}^{(2)} \frac{\partial v_{\alpha}^{(2)}}{\partial g_{\alpha}^{(2)}} \frac{\partial F_{3}^{(1,2,3)}}{\partial x_{\beta}^{(2)}}
$$

$28^{\text {th }}$ term,

$$
\begin{aligned}
& \left\langle-\delta\left(u^{(1)}-v^{(1)}\right) \delta\left(h^{(1)}-g^{(1)}\right) \delta\left(\theta^{(1)}-\varphi^{(1)}\right) \delta\left(c^{(1)}-\psi^{(1)}\right) \delta\left(u^{(2)}-v^{(2)}\right) \delta\left(h^{(2)}-g^{(2)}\right) \delta\left(\theta^{(2)}-\varphi^{(2)}\right)\right. \\
& \left.\delta\left(c^{(2)}-\psi^{(2)}\right) \delta\left(h^{(3)}-g^{(3)}\right) \delta\left(\theta^{(3)}-\varphi^{(3)}\right) \delta\left(c^{(3)}-\psi^{(3)}\right) \times \frac{\partial h_{\alpha}^{(3)} h_{\beta}^{(3)}}{\partial x_{\beta}^{(3)}} \frac{\partial}{\partial v_{\alpha}^{(3)}} \delta\left(u^{(3)}-v^{(3)}\right)\right\rangle \quad=-g_{\beta}^{(3)} \frac{\partial g_{\alpha}^{(3)}}{\partial v_{\alpha}^{(3)}} \frac{\partial F_{3}^{(1,2,3)}}{\partial x_{\beta}^{(3)}}
\end{aligned}
$$

and $33^{\text {rd }}$ term,

$$
\begin{aligned}
& \left\langle-\delta\left(u^{(1)}-v^{(1)}\right) \delta\left(h^{(1)}-g^{(1)}\right) \delta\left(\theta^{(1)}-\varphi^{(1)}\right) \delta\left(c^{(1)}-\psi^{(1)}\right) \delta\left(u^{(2)}-v^{(2)}\right) \delta\left(h^{(2)}-g^{(2)}\right) \delta\left(\theta^{(2)}-\varphi^{(2)}\right)\right. \\
& \left.\delta\left(c^{(2)}-\psi^{(2)}\right) \delta\left(u^{(3)}-v^{(3)}\right) \delta\left(\theta^{(3)}-\varphi^{(3)}\right) \delta\left(c^{(3)}-\psi^{(3)}\right) \times \frac{\partial u_{\alpha}^{(3)} h_{\beta}^{(3)}}{\partial x_{\beta}^{(3)}} \frac{\partial}{\partial g_{\alpha}^{(3)}} \delta\left(h^{(3)}-g^{(3)}\right)\right\rangle
\end{aligned}=-g_{\beta}^{(3)} \frac{\partial v_{\alpha}^{(3)}}{\partial g_{\alpha}^{(3)}} \frac{\partial F_{3}^{(1,2,3)}}{\partial x_{\beta}^{(3)}}
$$

Fourth term can be reduced as

$$
\begin{aligned}
& \left\langle-\delta\left(h^{(1)}-g^{(1)}\right) \delta\left(\theta^{(1)}-\varphi^{(1)}\right) \delta\left(c^{(1)}-\psi^{(1)}\right) \delta\left(u^{(2)}-v^{(2)}\right) \delta\left(h^{(2)}-g^{(2)}\right) \delta\left(\theta^{(2)}-\varphi^{(2)}\right) \delta\left(c^{(2)}-\psi^{(2)}\right)\right. \\
& \delta\left(u^{(3)}-v^{(3)}\right) \delta\left(h^{(3)}-g^{(3)}\right) \delta\left(\theta^{(3)}-\varphi^{(3)}\right) \delta\left(c^{(3)}-\psi^{(3)}\right) \times v \nabla^{2} u_{\alpha}^{(1)} \frac{\partial}{\partial v_{\alpha}^{(1)}} \delta\left(u^{(1)}-v^{(1)}\right) \\
& =-v \frac{\partial}{\partial v_{\alpha}^{(1)}}\left\langle\nabla ^ { 2 } u _ { \alpha } ^ { ( 1 ) } \left[\delta\left(u^{(1)}-v^{(1)}\right) \delta\left(h^{(1)}-g^{(1)}\right) \delta\left(\theta^{(1)}-\varphi^{(1)}\right) \delta\left(c^{(1)}-\psi^{(1)}\right) \delta\left(u^{(2)}-v^{(2)}\right) \delta\left(h^{(2)}-g^{(2)}\right)\right.\right. \\
& \left.\left.\delta\left(\theta^{(2)}-\varphi^{(2)}\right) \delta\left(c^{(2)}-\psi^{(2)}\right) \delta\left(u^{(3)}-v^{(3)}\right) \delta\left(h^{(3)}-g^{(3)}\right) \delta\left(\theta^{(3)}-\varphi^{(3)}\right) \delta\left(c^{(3)}-\psi^{(3)}\right)\right]\right\rangle \\
& =-v \frac{\partial}{\partial v_{\alpha}^{(1)}} \frac{\partial^{2}}{\partial x_{\beta}^{(1)} \partial x_{\beta}^{(1)}}\left\langleu _ { \alpha } ^ { ( 1 ) } \left[\delta\left(u^{(1)}-v^{(1)}\right) \delta\left(h^{(1)}-g^{(1)}\right) \delta\left(\theta^{(1)}-\varphi^{(1)}\right) \delta\left(c^{(1)}-\psi^{(1)}\right) \delta\left(u^{(2)}-v^{(2)}\right)\right.\right. \\
& \left.\left.\delta\left(h^{(2)}-g^{(2)}\right) \delta\left(\theta^{(2)}-\varphi^{(2)}\right) \delta\left(c^{(2)}-\psi^{(2)}\right) \delta\left(u^{(3)}-v^{(3)}\right) \delta\left(h^{(3)}-g^{(3)}\right) \delta\left(\theta^{(3)}-\varphi^{(3)}\right) \delta\left(c^{(3)}-\psi^{(3)}\right)\right]\right\rangle
\end{aligned}
$$

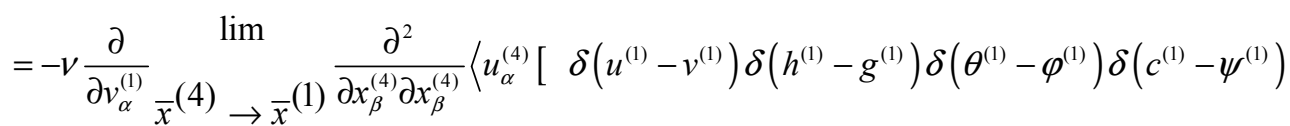

$$
\begin{aligned}
& \left.\left.\delta\left(u^{(2)}-v^{(2)}\right) \delta\left(h^{(2)}-g^{(2)}\right) \delta\left(\theta^{(2)}-\varphi^{(2)}\right) \delta\left(c^{(2)}-\psi^{(2)}\right) \delta\left(u^{(3)}-v^{(3)}\right) \delta\left(h^{(3)}-g^{(3)}\right) \delta\left(\theta^{(3)}-\varphi^{(3)}\right) \delta\left(c^{(3)}-\psi^{(3)}\right)\right]\right\rangle \\
& =-v \frac{\partial}{\partial v_{\alpha}^{(1)}} \lim _{\bar{x}}^{(4)} \rightarrow \bar{x}^{(1)} \frac{\partial^{2}}{\partial x_{\beta}^{(4)} \partial x_{\beta}^{(4)}}\left\langle\int u_{\alpha}^{(4)} \delta\left(u^{(4)}-v^{(4)}\right) \delta\left(h^{(4)}-g^{(4)}\right) \delta\left(\theta^{(4)}-\varphi^{(4)}\right) \delta\left(c^{(4)}-\psi^{(4)}\right)\right. \\
& \delta\left(u^{(3)}-v^{(3)}\right) \delta\left(h^{(3)}-g^{(3)}\right) \delta\left(\theta^{(3)}-\varphi^{(3)}\right) \delta\left(c^{(3)}-\psi^{(3)}\right) \delta\left(u^{(2)}-v^{(2)}\right) \delta\left(h^{(2)}-g^{(2)}\right) \delta\left(\theta^{(2)}-\varphi^{(2)}\right) \\
& \left.\delta\left(c^{(2)}-\psi^{(2)}\right) \delta\left(u^{(1)}-v^{(1)}\right) \delta\left(h^{(1)}-g^{(1)}\right) \delta\left(\theta^{(1)}-\varphi^{(1)}\right) \delta\left(c^{(1)}-\psi^{(1)}\right) d v^{(4)} d g^{(4)} d \varphi^{(4)} d \psi^{(4)}\right\rangle \\
& =-v \frac{\partial}{\partial v_{\alpha}^{(1)}} \lim _{\bar{x}}^{(4)} \rightarrow \bar{x}^{(1)} \frac{\partial^{2}}{\partial x_{\beta}^{(4)} \partial x_{\beta}^{(4)}} \int v_{\alpha}^{(4)} F_{4}^{(1,2,3,4)} d v^{(4)} d g^{(4)} d \varphi^{(4)} d \psi^{(4)}
\end{aligned}
$$


Similarly, $8^{\text {th }}, 10^{\text {th }}, 12^{\text {th }}, 17^{\text {th }}, 21^{\text {st }}, 23^{\text {rd }}, 25^{\text {th }}, 30^{\text {th }}, 34^{\text {th }}, 36^{\text {th }}$ and $38^{\text {th }}$ terms of right hand-side of equation (70) can be simplified as follows;

$$
\begin{gathered}
\left\langle-\delta\left(u^{(1)}-v^{(1)}\right) \delta\left(\theta^{(1)}-\varphi^{(1)}\right) \delta\left(c^{(1)}-\psi^{(1)}\right) \delta\left(u^{(2)}-v^{(2)}\right) \delta\left(h^{(2)}-g^{(2)}\right) \delta\left(\theta^{(2)}-\varphi^{(2)}\right) \delta\left(c^{(2)}-\psi^{(2)}\right)\right. \\
\left.\delta\left(u^{(3)}-v^{(3)}\right) \delta\left(h^{(3)}-g^{(3)}\right) \delta\left(\theta^{(3)}-\varphi^{(3)}\right) \delta\left(c^{(3)}-\psi^{(3)}\right) \times \lambda \nabla^{2} h_{\alpha}^{(1)} \frac{\partial}{\partial g_{\alpha}^{(1)}} \delta\left(h^{(1)}-g^{(1)}\right)\right\rangle \\
=-\lambda \frac{\partial}{\partial g_{\alpha}^{(1)}} \bar{x}^{(4)} \rightarrow \bar{x}^{(1)} \frac{\partial^{2}}{\partial x_{\beta}^{(4)} \partial x_{\beta}^{(4)}} \int g_{\alpha}^{(4)} F_{4}^{(1,2,3,4)} d v^{(4)} d g^{(4)} d \varphi^{(4)} d \psi^{(4)}
\end{gathered}
$$

$10^{\text {th }}$ term,

$$
\begin{gathered}
\left\langle-\delta\left(u^{(1)}-v^{(1)}\right) \delta\left(h^{(1)}-g^{(1)}\right) \delta\left(c^{(1)}-\psi^{(1)}\right) \delta\left(u^{(2)}-v^{(2)}\right) \delta\left(h^{(2)}-g^{(2)}\right) \delta\left(\theta^{(2)}-\varphi^{(2)}\right) \delta\left(c^{(2)}-\psi^{(2)}\right)\right. \\
\left.\delta\left(u^{(3)}-v^{(3)}\right) \delta\left(h^{(3)}-g^{(3)}\right) \delta\left(\theta^{(3)}-\varphi^{(3)}\right) \delta\left(c^{(3)}-\psi^{(3)}\right) \times \gamma^{2} \theta^{(1)} \frac{\partial}{\partial \varphi^{(1)}} \delta\left(\theta^{(1)}-\varphi^{(1)}\right)\right\rangle \\
=-\gamma \frac{\partial}{\partial \varphi^{(1)}} \overline{\bar{x}}^{(4)} \rightarrow \bar{x}^{(1)} \frac{\partial^{2}}{\partial x_{\beta}^{(4)} \partial x_{\beta}^{(4)}} \int \varphi^{(4)} F_{4}^{(1,2,3,4)} d v^{(4)} d g^{(4)} d \varphi^{(4)} d \psi^{(4)}
\end{gathered}
$$

$12^{\text {th }}$ term,

$$
\begin{gathered}
+\left\langle-\delta\left(u^{(1)}-v^{(1)}\right) \delta\left(h^{(1)}-g^{(1)}\right) \delta\left(\theta^{(1)}-\varphi^{(1)}\right) \delta\left(u^{(2)}-v^{(2)}\right) \delta\left(h^{(2)}-g^{(2)}\right) \delta\left(\theta^{(2)}-\varphi^{(2)}\right) \delta\left(c^{(2)}-\psi^{(2)}\right)\right. \\
\left.\delta\left(u^{(3)}-v^{(3)}\right) \delta\left(h^{(3)}-g^{(3)}\right) \delta\left(\theta^{(3)}-\varphi^{(3)}\right) \delta\left(c^{(3)}-\psi^{(3)}\right) \times D \nabla^{2} c^{(1)} \frac{\partial}{\partial \psi^{(1)}} \delta\left(c^{(1)}-\psi^{(1)}\right)\right\rangle \\
=-D \frac{\partial}{\partial \psi^{(1)}} \overline{\bar{x}}^{(4)} \rightarrow \bar{x}^{(1)} \frac{\partial^{2}}{\partial x_{\beta}^{(4)} \partial x_{\beta}^{(3)}} \int \psi^{(4)} F_{4}^{(1,2,3,4)} d v^{(4)} d g^{(4)} d \varphi^{(4)} d \psi^{(4)}
\end{gathered}
$$

$17^{\text {th }}$ term,

$$
\begin{gathered}
\left\langle-\delta\left(u^{(1)}-v^{(1)}\right) \delta\left(h^{(1)}-g^{(1)}\right) \delta\left(\theta^{(1)}-\varphi^{(1)}\right) \delta\left(c^{(1)}-\psi^{(1)}\right) \delta\left(h^{(2)}-g^{(2)}\right) \delta\left(\theta^{(2)}-\varphi^{(2)}\right) \delta\left(c^{(2)}-\psi^{(2)}\right)\right. \\
\left.\delta\left(u^{(3)}-v^{(3)}\right) \delta\left(h^{(3)}-g^{(3)}\right) \delta\left(\theta^{(3)}-\varphi^{(3)}\right) \delta\left(c^{(3)}-\psi^{(3)}\right) \times v \nabla^{2} u_{\alpha}^{(2)} \frac{\partial}{\partial v_{\alpha}^{(2)}} \delta\left(u^{(2)}-v^{(2)}\right)\right\rangle \\
=-v \frac{\partial}{\partial v_{\alpha}^{(2)}} \bar{x}^{(4)} \rightarrow \bar{x}^{(2)} \frac{\partial^{2}}{\partial x_{\beta}^{(4)} \partial x_{\beta}^{(4)}} \int v_{\alpha}^{(4)} F_{4}^{(1,2,3,4)} d v^{(4)} d g^{(4)} d \varphi^{(4)} d \psi^{(4)}
\end{gathered}
$$

$21^{\text {st }}$ term,

$$
\begin{gathered}
\left\langle-\delta\left(u^{(1)}-v^{(1)}\right) \delta\left(h^{(1)}-g^{(1)}\right) \delta\left(\theta^{(1)}-\varphi^{(1)}\right) \delta\left(c^{(1)}-\psi^{(1)}\right) \delta\left(u^{(2)}-v^{(2)}\right) \delta\left(\theta^{(2)}-\varphi^{(2)}\right) \delta\left(c^{(2)}-\psi^{(2)}\right)\right. \\
\left.\delta\left(u^{(3)}-v^{(3)}\right) \delta\left(h^{(3)}-g^{(3)}\right) \delta\left(\theta^{(3)}-\varphi^{(3)}\right) \delta\left(c^{(3)}-\psi^{(3)}\right) \times \lambda \nabla^{2} h_{\alpha}^{(2)} \frac{\partial}{\partial g_{\alpha}^{(2)}} \delta\left(h^{(2)}-g^{(2)}\right)\right\rangle \\
=-\lambda \frac{\partial}{\partial g_{\alpha}^{(2)}} \bar{x}^{(4)} \rightarrow \bar{x}^{(2)} \frac{\partial^{2}}{\partial x_{\beta}^{(4)} \partial x_{\beta}^{(4)}} \int g_{\alpha}^{(4)} F_{4}^{(1,2,3,4)} d v^{(4)} d g^{(4)} d \varphi^{(4)} d \psi^{(4)}
\end{gathered}
$$

$23^{\text {rd }}$ term, 


$$
\begin{gathered}
\left\langle-\delta\left(u^{(1)}-v^{(1)}\right) \delta\left(h^{(1)}-g^{(1)}\right) \delta\left(\theta^{(1)}-\varphi^{(1)}\right) \delta\left(c^{(1)}-\psi^{(1)}\right) \delta\left(u^{(2)}-v^{(2)}\right) \delta\left(h^{(2)}-g^{(2)}\right) \delta\left(c^{(2)}-\psi^{(2)}\right)\right. \\
\left.\delta\left(u^{(3)}-v^{(3)}\right) \delta\left(h^{(3)}-g^{(3)}\right) \delta\left(\theta^{(3)}-\varphi^{(3)}\right) \delta\left(c^{(3)}-\psi^{(3)}\right) \times \nabla^{2} \theta^{(2)} \frac{\partial}{\partial \varphi^{(2)}} \delta\left(\theta^{(2)}-\varphi^{(2)}\right)\right\rangle \\
=-\gamma \frac{\partial}{\partial \varphi^{(2)}} \bar{x}^{(4)} \rightarrow \bar{x}^{(2)} \frac{\partial^{2}}{\partial x_{\beta}^{(4)} \partial x_{\beta}^{(4)}} \int \varphi^{(4)} F_{4}^{(1,2,3,4)} d v^{(4)} d g^{(4)} d \varphi^{(4)} d \psi^{(4)}
\end{gathered}
$$

$25^{\text {th }}$ term,

$$
\begin{gathered}
\left\langle-\delta\left(u^{(1)}-v^{(1)}\right) \delta\left(h^{(1)}-g^{(1)}\right) \delta\left(\theta^{(1)}-\varphi^{(1)}\right) \delta\left(c^{(1)}-\psi^{(1)}\right) \delta\left(u^{(2)}-v^{(2)}\right) \delta\left(h^{(2)}-g^{(2)}\right) \delta\left(\theta^{(2)}-\varphi^{(2)}\right)\right. \\
\left.\delta\left(u^{(3)}-v^{(3)}\right) \delta\left(h^{(3)}-g^{(3)}\right) \delta\left(\theta^{(3)}-\varphi^{(3)}\right) \delta\left(c^{(3)}-\psi^{(3)}\right) \times D \nabla^{2} c^{(2)} \frac{\partial}{\partial \psi^{(2)}} \delta\left(c^{(2)}-\psi^{(2)}\right)\right\rangle \\
=-D \frac{\partial}{\partial \psi^{(2)}} \bar{x}^{(4)} \rightarrow \bar{x}^{(2)} \frac{\partial^{2}}{\partial x_{\beta}^{(4)} \partial x_{\beta}^{(4)}} \int \psi^{(4)} F_{4}^{(1,2,3,4)} d v^{(4)} d g^{(4)} d \varphi^{(4)} d \psi^{(4)}
\end{gathered}
$$

$30^{\text {th }}$ term,

$$
\begin{gathered}
\left\langle-\delta\left(u^{(1)}-v^{(1)}\right) \delta\left(h^{(1)}-g^{(1)}\right) \delta\left(\theta^{(1)}-\varphi^{(1)}\right) \delta\left(c^{(1)}-\psi^{(1)}\right) \delta\left(u^{(2)}-v^{(2)}\right) \delta\left(h^{(2)}-g^{(2)}\right) \delta\left(\theta^{(2)}-\varphi^{(2)}\right)\right. \\
\left.\delta\left(c^{(2)}-\psi^{(2)}\right) \delta\left(h^{(3)}-g^{(3)}\right) \delta\left(\theta^{(3)}-\varphi^{(3)}\right) \delta\left(c^{(3)}-\psi^{(3)}\right) \times \nabla^{2} u_{\alpha}^{(3)} \frac{\partial}{\partial v_{\alpha}^{(3)}} \delta\left(u^{(3)}-v^{(3)}\right)\right\rangle \\
=-v \frac{\partial}{\partial v_{\alpha}^{(3)}} \bar{x}^{(4)} \rightarrow \bar{x}^{(3)} \frac{\partial^{2}}{\partial x_{\beta}^{(4)} \partial x_{\beta}^{(4)}} \int v_{\alpha}^{(4)} F_{4}^{(1,2,3,4)} d v^{(4)} d g^{(4)} d \varphi^{(4)} d \psi^{(4)}
\end{gathered}
$$

$34^{\text {th }}$ term,

$$
\begin{gathered}
\left\langle-\delta\left(u^{(1)}-v^{(1)}\right) \delta\left(h^{(1)}-g^{(1)}\right) \delta\left(\theta^{(1)}-\varphi^{(1)}\right) \delta\left(c^{(1)}-\psi^{(1)}\right) \delta\left(u^{(2)}-v^{(2)}\right) \delta\left(h^{(2)}-g^{(2)}\right) \delta\left(\theta^{(2)}-\varphi^{(2)}\right)\right. \\
\left.\delta\left(c^{(2)}-\psi^{(2)}\right) \delta\left(u^{(3)}-v^{(3)}\right) \delta\left(\theta^{(3)}-\varphi^{(3)}\right) \delta\left(c^{(3)}-\psi^{(3)}\right) \times \lambda \nabla^{2} h_{\alpha}^{(3)} \frac{\partial}{\partial g_{\alpha}^{(3)}} \delta\left(h^{(3)}-g^{(3)}\right)\right\rangle \\
=-\lambda \frac{\partial}{\partial g_{\alpha}^{(3)}} \bar{x}^{(4)} \rightarrow \bar{x}^{(3)} \frac{\partial^{2}}{\partial x_{\beta}^{(4)} \partial x_{\beta}^{(4)}} \int g_{\alpha}^{(4)} F_{4}^{(1,2,3,4)} d v^{(4)} d g^{(4)} d \varphi^{(4)} d \psi^{(4)}
\end{gathered}
$$

$36^{\text {th }}$ term,

$$
\begin{gathered}
\left\langle-\delta\left(u^{(1)}-v^{(1)}\right) \delta\left(h^{(1)}-g^{(1)}\right) \delta\left(\theta^{(1)}-\varphi^{(1)}\right) \delta\left(c^{(1)}-\psi^{(1)}\right) \delta\left(u^{(2)}-v^{(2)}\right) \delta\left(h^{(2)}-g^{(2)}\right) \delta\left(\theta^{(2)}-\varphi^{(2)}\right)\right. \\
\left.\delta\left(c^{(2)}-\psi^{(2)}\right) \delta\left(u^{(3)}-v^{(3)}\right) \delta\left(h^{(3)}-g^{(3)}\right) \delta\left(c^{(3)}-\psi^{(3)}\right) \times \nabla^{2} \theta^{(3)} \frac{\partial}{\partial \varphi^{(3)}} \delta\left(\theta^{(3)}-\varphi^{(3)}\right)\right\rangle \\
=-\gamma \frac{\partial}{\partial \varphi^{(3)}} \bar{x}^{(4)} \rightarrow \bar{x}^{(3)} \frac{\partial^{2}}{\partial x_{\beta}^{(4)} \partial x_{\beta}^{(4)}} \int \varphi^{(4)} F_{4}^{(1,2,3,4)} d v^{(4)} d g^{(4)} d \varphi^{(4)} d \psi^{(4)}
\end{gathered}
$$

$38^{\text {th }}$ term,

$$
\begin{aligned}
& \left\langle-\delta\left(u^{(1)}-v^{(1)}\right) \delta\left(h^{(1)}-g^{(1)}\right) \delta\left(\theta^{(1)}-\varphi^{(1)}\right) \delta\left(c^{(1)}-\psi^{(1)}\right) \delta\left(u^{(2)}-v^{(2)}\right) \delta\left(h^{(2)}-g^{(2)}\right) \delta\left(\theta^{(2)}-\varphi^{(2)}\right)\right. \\
& \left.\delta\left(c^{(2)}-\psi^{(2)}\right) \delta\left(u^{(3)}-v^{(3)}\right) \delta\left(h^{(3)}-g^{(3)}\right) \delta\left(\theta^{(3)}-\varphi^{(3)}\right) \times D \nabla^{2} c^{(3)} \frac{\partial}{\partial \psi^{(3)}} \delta\left(c^{(3)}-\psi^{(3)}\right)\right\rangle
\end{aligned}
$$




$$
=-D \frac{\partial}{\partial \psi^{(3)}} \lim _{\bar{x}^{(4)} \rightarrow \bar{x}^{(3)}} \frac{\partial^{2}}{\partial x_{\beta}^{(4)} \partial x_{\beta}^{(4)}} \int \psi^{(4)} F_{4}^{(1,2,3,4)} d v^{(4)} d g^{(4)} d \varphi^{(4)} d \psi^{(4)}
$$

We reduce the third term of right hand side of equation (17),

$$
\begin{gathered}
+\left\langle\delta\left(h^{(1)}-g^{(1)}\right) \delta\left(\theta^{(1)}-\varphi^{(1)}\right) \delta\left(c^{(1)}-\psi^{(1)}\right) \delta\left(u^{(2)}-v^{(2)}\right) \delta\left(h^{(2)}-g^{(2)}\right) \delta\left(\theta^{(2)}-\varphi^{(2)}\right) \delta\left(c^{(2)}-\psi^{(2)}\right)\right. \\
\delta\left(u^{(3)}-v^{(3)}\right) \delta\left(h^{(3)}-g^{(3)}\right) \delta\left(\theta^{(3)}-\varphi^{(3)}\right) \delta\left(c^{(3)}-\psi^{(3)}\right) \times \frac{1}{4 \pi} \frac{\partial}{\partial x_{\alpha}^{(1)}} \int\left[\frac{\partial u_{\alpha}^{(1)}}{\partial x_{\beta}^{(1)}} \frac{\partial u_{\beta}^{(1)}}{\partial x_{\alpha}^{(1)}}-\frac{\partial h_{\alpha}^{(1)}}{\partial x_{\beta}^{(1)}} \frac{\partial h_{\beta}^{(1)}}{\partial x_{\alpha}^{(1)}}\right] \times \frac{\partial}{\left|\bar{x}^{\prime \prime \prime}-\bar{x}\right|} \frac{\partial v_{\alpha}^{(1)}}{\left.\delta\left(u^{(1)}-v^{(1)}\right)\right\rangle} \\
=\frac{\partial}{\partial v_{\alpha}^{(1)}}\left[\frac{1}{4 \pi} \int \frac{\partial}{\partial x_{\alpha}^{(1)}}\left(\frac{1}{\left|\bar{x}^{(4)}-\bar{x}^{(1)}\right|}\right)\left(\frac{\partial v_{\alpha}^{(4)}}{\partial x_{\beta}^{(4)}} \frac{\partial v_{\beta}^{(4)}}{\partial x_{\alpha}^{(4)}}-\frac{\partial g_{\alpha}^{(4)}}{\partial x_{\beta}^{(4)}} \frac{\partial g_{\beta}^{(4)}}{\partial x_{\alpha}^{(4)}}\right) F_{4}^{(1,2,3,4)} d x^{(4)} d v^{(4)} d g^{(4)} d \varphi^{(4)} d \psi^{(4)}\right]
\end{gathered}
$$

$16^{\text {th }}$ term,

$$
\begin{aligned}
& \left\langle\delta\left(u^{(1)}-v^{(1)}\right) \delta\left(h^{(1)}-g^{(1)}\right) \delta\left(\theta^{(1)}-\varphi^{(1)}\right) \delta\left(c^{(1)}-\psi^{(1)}\right) \delta\left(h^{(2)}-g^{(2)}\right) \delta\left(\theta^{(2)}-\varphi^{(2)}\right) \delta\left(c^{(2)}-\psi^{(2)}\right)\right. \\
& \delta\left(u^{(3)}-v^{(3)}\right) \delta\left(h^{(3)}-g^{(3)}\right) \delta\left(\theta^{(3)}-\varphi^{(3)}\right) \delta\left(c^{(3)}-\psi^{(3)}\right) \\
& \left.\times \frac{1}{4 \pi} \frac{\partial}{\partial x_{\alpha}^{(2)}} \int\left[\frac{\partial u_{\alpha}^{(2)}}{\partial x_{\beta}^{(2)}} \frac{\partial u_{\beta}^{(2)}}{\partial x_{\alpha}^{(2)}}-\frac{\partial h_{\alpha}^{(2)}}{\partial x_{\beta}^{(2)}} \frac{\partial h_{\beta}^{(2)}}{\partial x_{\alpha}^{(2)}}\right] \times \frac{d \bar{x}^{\prime \prime \prime}}{\left|\bar{x}^{\prime \prime \prime}-\bar{x}^{\prime}\right|} \frac{\partial}{\partial v_{\alpha}^{(2)}} \delta\left(u^{(2)}-v^{(2)}\right)\right\rangle \\
= & \frac{\partial}{\partial v_{\alpha}^{(2)}}\left[\frac{1}{4 \pi} \int \frac{\partial}{\partial x_{\alpha}^{(2)}}\left(\frac{1}{\left|\bar{x}^{(4)}-\bar{x}^{(2)}\right|}\right)\left(\frac{\partial v_{\alpha}^{(4)}}{\partial x_{\beta}^{(4)}} \frac{\partial v_{\beta}^{(4)}}{\partial x_{\alpha}^{(4)}}-\frac{\partial g_{\alpha}^{(4)}}{\partial x_{\beta}^{(4)}} \frac{\partial g_{\beta}^{(4)}}{\partial x_{\alpha}^{(4)}}\right) F_{4}^{(1,2,3,4)} d x^{(4)} d v^{(4)} d g^{(4)} d \varphi^{(4)} d \psi^{(4)}\right]
\end{aligned}
$$

Similarly, $29^{\text {th }}$ term,

$$
\begin{gathered}
\left\langle\delta\left(u^{(1)}-v^{(1)}\right) \delta\left(h^{(1)}-g^{(1)}\right) \delta\left(\theta^{(1)}-\varphi^{(1)}\right) \delta\left(c^{(1)}-\psi^{(1)}\right) \delta\left(u^{(2)}-v^{(2)}\right) \delta\left(h^{(2)}-g^{(2)}\right)\right. \\
\delta\left(\theta^{(2)}-\varphi^{(2)}\right) \delta\left(c^{(2)}-\psi^{(2)}\right) \delta\left(h^{(3)}-g^{(3)}\right) \delta\left(\theta^{(3)}-\varphi^{(3)}\right) \delta\left(c^{(3)}-\psi^{(3)}\right) \\
\left.\times \frac{1}{4 \pi} \frac{\partial}{\partial x_{\alpha}^{(3)}} \int\left[\frac{\partial u_{\alpha}^{(3)}}{\partial x_{\beta}^{(3)}} \frac{\partial u_{\beta}^{(3)}}{\partial x_{\alpha}^{(3)}}-\frac{\partial h_{\alpha}^{(3)}}{\partial x_{\beta}^{(3)}} \frac{\partial h_{\beta}^{(3)}}{\partial x_{\alpha}^{(3)}}\right] \frac{d \bar{x}^{\prime \prime \prime}}{\left|\bar{x}^{\prime \prime \prime}-\bar{x}^{\prime \prime}\right|} \frac{\partial}{\partial v_{\alpha}^{(3)}} \delta\left(u^{(3)}-v^{(3)}\right)\right\rangle \\
=\frac{\partial}{\partial v_{\alpha}^{(3)}}\left[\frac{1}{4 \pi} \int \frac{\partial}{\partial x_{\alpha}^{(3)}}\left(\frac{1}{\left|\bar{x}^{(4)}-\bar{x}^{(3)}\right|}\right)\left(\frac{\partial v_{\alpha}^{(4)}}{\partial x_{\beta}^{(4)}} \frac{\partial v_{\beta}^{(4)}}{\partial x_{\alpha}^{(4)}}-\frac{\partial g_{\alpha}^{(4)}}{\partial x_{\beta}^{(4)}} \frac{\partial g_{\beta}^{(4)}}{\partial x_{\alpha}^{(4)}}\right) F_{4}^{(1,2,3,4)} d x^{(4)} d v^{(4)} d g^{(4)} d \varphi^{(4)} d \psi^{(4)}\right]
\end{gathered}
$$

$5^{\text {th }}$ term of right hand side of equation (17),

$$
\begin{gathered}
\left\langle-\delta\left(h^{(1)}-g^{(1)}\right) \delta\left(\theta^{(1)}-\varphi^{(1)}\right) \delta\left(c^{(1)}-\psi^{(1)}\right) \delta\left(u^{(2)}-v^{(2)}\right) \delta\left(h^{(2)}-g^{(2)}\right) \delta\left(\theta^{(2)}-\varphi^{(2)}\right) \delta\left(c^{(2)}-\psi^{(2)}\right)\right. \\
\left.\delta\left(u^{(3)}-v^{(3)}\right) \delta\left(h^{(3)}-g^{(3)}\right) \delta\left(\theta^{(3)}-\varphi^{(3)}\right) \delta\left(c^{(3)}-\psi^{(3)}\right) \times f\left(u_{\alpha}^{(1)}-v_{\alpha}^{(1)}\right) \frac{\partial}{\partial v_{\alpha}^{(1)}} \delta\left(u^{(1)}-v^{(1)}\right)\right\rangle \\
=-\left\langlef ( u _ { \alpha } ^ { ( 1 ) } - v _ { \alpha } ^ { ( 1 ) } ) \frac { \partial } { \partial v _ { \alpha } ^ { ( 1 ) } } \left[\delta\left(u^{(1)}-v^{(1)}\right) \delta\left(h^{(1)}-g^{(1)}\right) \delta\left(\theta^{(1)}-\varphi^{(1)}\right) \delta\left(c^{(1)}-\psi^{(1)}\right) \delta\left(u^{(2)}-v^{(2)}\right)\right.\right. \\
\left.\left.\delta\left(h^{(2)}-g^{(2)}\right) \delta\left(\theta^{(2)}-\varphi^{(2)}\right) \delta\left(c^{(2)}-\psi^{(2)}\right) \delta\left(u^{(3)}-v^{(3)}\right) \delta\left(h^{(3)}-g^{(3)}\right) \delta\left(\theta^{(3)}-\varphi^{(3)}\right) \delta\left(c^{(3)}-\psi^{(3)}\right)\right]\right\rangle \\
=-f\left(u_{\alpha}^{(1)}-v_{\alpha}^{(1)}\right) \frac{\partial}{\partial v_{\alpha}^{(1)}}\left\langle\delta\left(u^{(1)}-v^{(1)}\right) \delta\left(h^{(1)}-g^{(1)}\right) \delta\left(\theta^{(1)}-\varphi^{(1)}\right) \delta\left(c^{(1)}-\psi^{(1)}\right) \delta\left(u^{(2)}-v^{(2)}\right) \quad=-f\left(u_{\alpha}^{(1)}-v_{\alpha}^{(1)}\right) \frac{\partial}{\partial v_{\alpha}^{(1)}} F_{3}^{(1,2,3)}\right. \\
\left.\delta\left(h^{(2)}-g^{(2)}\right) \delta\left(\theta^{(2)}-\varphi^{(2)}\right) \delta\left(c^{(2)}-\psi^{(2)}\right) \delta\left(u^{(3)}-v^{(3)}\right) \delta\left(h^{(3)}-g^{(3)}\right) \delta\left(\theta^{(3)}-\varphi^{(3)}\right) \delta\left(c^{(3)}-\psi^{(3)}\right)\right\rangle
\end{gathered}
$$

Similarly, $18^{\text {th }}, 33^{\text {rd }}$ and $34^{\text {th }}$ terms of right hand side of equation (17), 
$18^{\text {th }}$ term,

$$
\begin{gathered}
\left\langle-\delta\left(u^{(1)}-v^{(1)}\right) \delta\left(h^{(1)}-g^{(1)}\right) \delta\left(\theta^{(1)}-\varphi^{(1)}\right) \delta\left(c^{(1)}-\psi^{(1)}\right) \delta\left(h^{(2)}-g^{(2)}\right) \delta\left(\theta^{(2)}-\varphi^{(2)}\right) \delta\left(c^{(2)}-\psi^{(2)}\right)\right. \\
\left.\delta\left(u^{(3)}-v^{(3)}\right) \delta\left(h^{(3)}-g^{(3)}\right) \delta\left(\theta^{(3)}-\varphi^{(3)}\right) \delta\left(c^{(3)}-\psi^{(3)}\right) \times f\left(u_{\alpha}^{(2)}-v_{\alpha}^{(2)}\right) \frac{\partial}{\partial v_{\alpha}^{(2)}} \delta\left(u^{(2)}-v^{(2)}\right)\right\rangle \\
=-f\left(u_{\alpha}^{(2)}-v_{\alpha}^{(2)}\right) \frac{\partial}{\partial v_{\alpha}^{(2)}} F_{3}^{(1,2,3)}
\end{gathered}
$$

$31^{\text {st }}$ term,

$\begin{aligned} & \left\langle-\delta\left(u^{(1)}-v^{(1)}\right) \delta\left(h^{(1)}-g^{(1)}\right) \delta\left(\theta^{(1)}-\varphi^{(1)}\right) \delta\left(c^{(1)}-\psi^{(1)}\right) \delta\left(u^{(2)}-v^{(2)}\right) \delta\left(h^{(2)}-g^{(2)}\right) \delta\left(\theta^{(2)}-\varphi^{(2)}\right)\right. \\ & \left.\delta\left(c^{(2)}-\psi^{(2)}\right) \delta\left(h^{(3)}-g^{(3)}\right) \delta\left(\theta^{(3)}-\varphi^{(3)}\right) \delta\left(c^{(3)}-\psi^{(3)}\right) \times f\left(u_{\alpha}^{(3)}-v_{\alpha}^{(3)}\right) \frac{\partial}{\partial v_{\alpha}^{(3)}} \delta\left(u^{(3)}-v^{(3)}\right)\right\rangle\end{aligned}=-f\left(u_{\alpha}^{(3)}-v_{\alpha}^{(3)}\right) \frac{\partial}{\partial v_{\alpha}^{(3)}} F_{3}^{(1,2,3)}$

$13^{\text {th }}$ term of Equation (17)

$$
\begin{aligned}
& \delta\left(u^{(1)}-v^{(1)}\right) \delta\left(h^{(1)}-g^{(1)}\right) \delta\left(\theta^{(1)}-\varphi^{(1)}\right) \delta\left(u^{(2)}-v^{(2)}\right) \delta\left(h^{(2)}-g^{(2)}\right) \delta\left(\theta^{(2)}-\varphi^{(2)}\right) \delta\left(c^{(2)}-\psi^{(2)}\right) \\
& \left.\delta\left(u^{(3)}-v^{(3)}\right) \delta\left(h^{(3)}-g^{(3)}\right) \delta\left(\theta^{(3)}-\varphi^{(3)}\right) \delta\left(c^{(3)}-\psi^{(3)}\right) \times R c^{(1)} \frac{\partial}{\partial \psi^{(1)}} \delta\left(c^{(1)}-\psi^{(1)}\right)\right\rangle=R \psi^{(1)} \frac{\partial}{\partial \psi^{(1)}} F_{3}^{(1,2,3)}
\end{aligned}
$$

$26^{\text {th }}$ term of Equation (17)

$$
\begin{aligned}
& \delta\left(u^{(1)}-v^{(1)}\right) \delta\left(h^{(1)}-g^{(1)}\right) \delta\left(\theta^{(1)}-\varphi^{(1)}\right) \delta\left(c^{(1)}-\psi^{(1)}\right) \delta\left(u^{(2)}-v^{(2)}\right) \delta\left(h^{(2)}-g^{(2)}\right) \delta\left(\theta^{(2)}-\varphi^{(2)}\right) \\
& \left.\delta\left(u^{(3)}-v^{(3)}\right) \delta\left(h^{(3)}-g^{(3)}\right) \delta\left(\theta^{(3)}-\varphi^{(3)}\right) \delta\left(c^{(3)}-\psi^{(3)}\right) \times R c^{(2)} \frac{\partial}{\partial \psi^{(2)}} \delta\left(c^{(2)}-\psi^{(2)}\right)\right\rangle=R \psi^{(2)} \frac{\partial}{\partial \psi^{(2)}} F_{3}^{(1,2,3)}
\end{aligned}
$$

$39^{\text {th }}$ term of Equation (17)

$$
\begin{aligned}
& \delta\left(u^{(1)}-v^{(1)}\right) \delta\left(h^{(1)}-g^{(1)}\right) \delta\left(\theta^{(1)}-\varphi^{(1)}\right) \delta\left(c^{(1)}-\psi^{(1)}\right) \delta\left(u^{(2)}-v^{(2)}\right) \delta\left(h^{(2)}-g^{(2)}\right) \delta\left(\theta^{(2)}-\varphi^{(2)}\right) \\
& \left.\delta\left(u^{(3)}-v^{(3)}\right) \delta\left(h^{(3)}-g^{(3)}\right) \delta\left(\theta^{(3)}-\varphi^{(3)}\right) \times R c^{(3)} \frac{\partial}{\partial \psi^{(3)}} \delta\left(c^{(3)}-\psi^{(3)}\right)\right\rangle=R \psi^{(3)} \frac{\partial}{\partial \psi^{(3)}} F_{3}^{(1,2,3)}
\end{aligned}
$$

\section{Results}

Substituting the results (18) - (59) in equation (20) we get the transport equation for three- point distribution function $F_{3}^{(1,2,3)}(v, g, \varphi, \psi)$ in MHD turbulent flow for velocity, magnetic temperature and concentration in presence of dust particles undergoing a first order reaction as

$$
\begin{aligned}
& \frac{\partial F_{3}^{(1,2,3)}}{\partial t}+\left(v_{\beta}^{(1)} \frac{\partial}{\partial x_{\beta}^{(1)}}+v_{\beta}^{(2)} \frac{\partial}{\partial x_{\beta}^{(2)}} v_{\beta}^{(3)} \frac{\partial}{\partial x_{\beta}^{(3)}}\right) F_{3}^{(1,2,3)}+\left[g_{\beta}^{(1)}\left(\frac{\partial g_{\alpha}^{(1)}}{\partial v_{\alpha}^{(1)}}+\frac{\partial v_{\alpha}^{(1)}}{\partial g_{\alpha}^{(1)}}\right) \frac{\partial}{\partial x_{\beta}^{(1)}}\right. \\
& \left.+g_{\beta}^{(2)}\left(\frac{\partial g_{\alpha}^{(2)}}{\partial v_{\alpha}^{(2)}}+\frac{\partial v_{\alpha}^{(2)}}{\partial g_{\alpha}^{(2)}}\right) \frac{\partial}{\partial x_{\beta}^{(2)}}+g_{\beta}^{(3)}\left(\frac{\partial g_{\alpha}^{(3)}}{\partial v_{\alpha}^{(3)}}+\frac{\partial v_{\alpha}^{(3)}}{\partial g_{\alpha}^{(3)}}\right) \frac{\partial}{\partial x_{\beta}^{(3)}}\right] F_{3}^{(1,2,3)}
\end{aligned}
$$

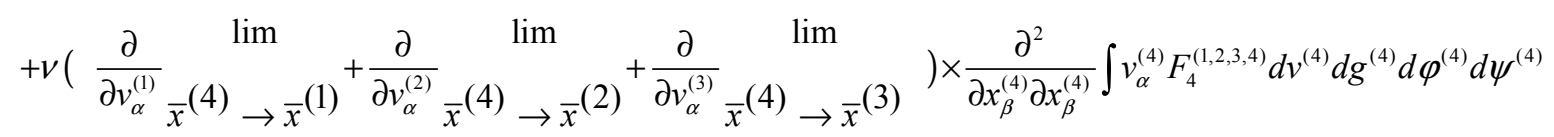

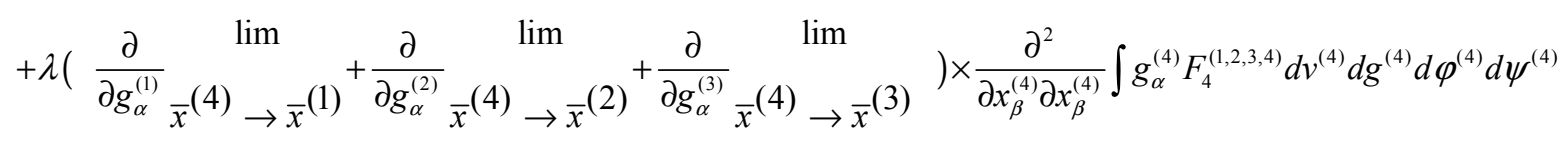

$$
\begin{aligned}
& +\gamma\left(\frac{\partial}{\partial \varphi^{(1)}}{ }_{\bar{x}^{(4)} \rightarrow \bar{x}^{(1)}}+\frac{\partial}{\partial \varphi^{(2)}} \lim _{\bar{x}}{ }^{(4)} \rightarrow \bar{x}^{(2)}+\frac{\partial}{\partial \varphi^{(3)}} \lim _{\bar{x}}{ }^{(4)} \bar{x}^{(3)}\right) \times \frac{\partial^{2}}{\partial x_{\beta}^{(4)} \partial x_{\beta}^{(4)}} \int \varphi^{(4)} F_{4}^{(1,2,3,4)} d v^{(4)} d g^{(4)} d \varphi^{(4)} d \psi^{(4)}
\end{aligned}
$$




$$
\begin{gathered}
+D\left(\frac{\partial}{\partial \psi^{(1)}} \bar{x}^{(4)} \rightarrow \bar{x}^{(1)}+\frac{\partial}{\partial \psi^{(2)}} \bar{x}^{(4)} \rightarrow \bar{x}^{(2)}+\frac{\partial}{\partial \psi^{(3)}} \bar{x}^{(4)} \rightarrow \bar{x}^{(3)}\right) \times \frac{\partial^{2}}{\partial x_{\beta}^{(4)} \partial x_{\beta}^{(4)}} \int \psi^{(4)} F_{4}^{(1,2,3,4)} d v^{(4)} d g^{(4)} d \varphi^{(4)} d \psi^{(4)} \\
-\left[\frac{\partial}{\partial v_{\alpha}^{(1)}}\left\{\frac{1}{4 \pi} \int \frac{\partial}{\partial x_{\alpha}^{(1)}}\left(\frac{1}{\left|\bar{x}^{(4)}-\bar{x}^{(1)}\right|}\right)\right\}+\frac{\partial}{\partial v_{\alpha}^{(2)}}\left\{\frac{1}{4 \pi} \int \frac{\partial}{\partial x_{\alpha}^{(2)}}\left(\frac{1}{\left|\bar{x}^{(4)}-\bar{x}^{(2)}\right|}\right)\right\}+\right. \\
\left.\frac{\partial}{\partial v_{\alpha}^{(3)}}\left\{\frac{1}{4 \pi} \int \frac{\partial}{\partial x_{\alpha}^{(3)}}\left(\frac{1}{\left|\bar{x}^{(4)}-\bar{x}^{(3)}\right|}\right)\right\} \times\left(\frac{\partial v_{\alpha}^{(4)}}{\partial x_{\beta}^{(4)}} \frac{\partial v_{\beta}^{(4)}}{\partial x_{\alpha}^{(4)}}-\frac{\partial g_{\alpha}^{(4)}}{\partial x_{\beta}^{(4)}} \frac{\partial g_{\beta}^{(4)}}{\partial x_{\alpha}^{(4)}}\right) F_{4}^{(1,2,3,4)} \times d x^{(4)} d v^{(4)} d g^{(4)} d \varphi^{(4)} d \psi^{(4)}\right] \\
+\left[f\left(u_{\alpha}^{(1)}-v_{\alpha}^{(1)}\right) \frac{\partial}{\partial v_{\alpha}^{(1)}}+f\left(u_{\alpha}^{(2)}-v_{\alpha}^{(2)}\right) \frac{\partial}{\partial v_{\alpha}^{(2)}}+f\left(u_{\alpha}^{(3)}-v_{\alpha}^{(3)}\right) \frac{\partial}{\partial v_{\alpha}^{(3)}}\right] F_{3}^{(1,2,3)} \\
-R\left(\psi^{(1)} \frac{\partial}{\partial \psi^{(1)}}+\psi^{(2)} \frac{\partial}{\partial \psi^{(2)}}+\psi^{(3)} \frac{\partial}{\partial \psi^{(3)}}\right) F_{3}^{(1,2,3)}=0
\end{gathered}
$$

Continuing this way, we can derive the equations for evolution of $F_{4}^{(1,2,3,4)}, F_{5}^{(1,2,3,4,5)}$ and so on. Logically it is possible to have an equation for every $F_{n}(n$ is an integer) but the system of equations so obtained is not closed. Certain approximations will be required thus obtained.

\section{Discussions}

If $\mathrm{R}=0$,i.e the reaction rate is absent, the transport equation for three- point distribution function in MHD turbulent flow (60) becomes

$$
\begin{gathered}
\frac{\partial F_{3}^{(1,2,3)}}{\partial t}+\left(v_{\beta}^{(1)} \frac{\partial}{\partial x_{\beta}^{(1)}}+v_{\beta}^{(2)} \frac{\partial}{\partial x_{\beta}^{(2)}} v_{\beta}^{(3)} \frac{\partial}{\partial x_{\beta}^{(3)}}\right) F_{3}^{(1,2,3)}+\left[g_{\beta}^{(1)}\left(\frac{\partial g_{\alpha}^{(1)}}{\partial v_{\alpha}^{(1)}}+\frac{\partial v_{\alpha}^{(1)}}{\partial g_{\alpha}^{(1)}}\right) \frac{\partial}{\partial x_{\beta}^{(1)}}\right. \\
\left.+g_{\beta}^{(2)}\left(\frac{\partial g_{\alpha}^{(2)}}{\partial v_{\alpha}^{(2)}}+\frac{\partial v_{\alpha}^{(2)}}{\partial g_{\alpha}^{(2)}}\right) \frac{\partial}{\partial x_{\beta}^{(2)}}+g_{\beta}^{(3)}\left(\frac{\partial g_{\alpha}^{(3)}}{\partial v_{\alpha}^{(3)}}+\frac{\partial v_{\alpha}^{(3)}}{\partial g_{\alpha}^{(3)}}\right) \frac{\partial}{\partial x_{\beta}^{(3)}}\right] F_{3}^{(1,2,3)} \\
+v\left(\frac{\partial}{\partial v_{\alpha}^{(1)}} \bar{x}^{(4)} \rightarrow \bar{x}^{(1)}+\frac{\partial}{\partial v_{\alpha}^{(2)}} \bar{x}^{(4)} \rightarrow \bar{x}^{(2)}+\frac{\partial}{\partial v_{\alpha}^{(3)}} \bar{x}^{(4)} \rightarrow \bar{x}^{(3)}\right) \times \frac{\partial^{2}}{\partial x_{\beta}^{(4)} \partial x_{\beta}^{(4)}} \int v_{\alpha}^{(4)} F_{4}^{(1,2,3,4)} d v^{(4)} d g^{(4)} d \varphi^{(4)} d \psi^{(4)} \\
+\lambda\left(\frac{\partial}{\partial g_{\alpha}^{(1)}} \bar{x}^{(4)} \rightarrow \bar{x}^{(1)}+\frac{\partial}{\partial g_{\alpha}^{(2)}} \bar{x}^{(4)} \rightarrow \bar{x}^{(2)}+\frac{\partial}{\partial g_{\alpha}^{(3)}} \bar{x}^{(4)} \rightarrow \bar{x}^{(3)}\right) \times \frac{\partial^{2}}{\partial x_{\beta}^{(4)} \partial x_{\beta}^{(4)}} \int g_{\alpha}^{(4)} F_{4}^{(1,2,3,4)} d v^{(4)} d g^{(4)} d \varphi^{(4)} d \psi^{(4)} \\
+\gamma\left(\frac{\partial}{\partial \varphi^{(1)}} \bar{x}^{(4)} \rightarrow \bar{x}^{(1)}+\frac{\partial}{\partial \varphi^{(2)}} \bar{x}^{(4)} \rightarrow \bar{x}^{(2)}+\frac{\partial}{\partial \varphi^{(3)}} \bar{x}^{(4)} \rightarrow \bar{x}^{(3)}\right) \times \frac{\partial^{2}}{\partial x_{\beta}^{(4)} \partial x_{\beta}^{(4)}} \int \varphi^{(4)} F_{4}^{(1,2,3,4)} d v^{(4)} d g^{(4)} d \varphi^{(4)} d \psi^{(4)} \\
+D\left(\frac{\partial}{\partial \psi^{(1)}} \overline{l i m}^{(4)} \rightarrow \bar{x}^{(1)}+\frac{\partial}{\partial \psi^{(2)}} \bar{x}^{(4)} \rightarrow \bar{x}^{(2)}+\frac{\partial}{\partial \psi^{(3)}} \bar{x}^{(4)} \rightarrow \bar{x}^{(3)}\right) \times \frac{\partial^{2}}{\partial x_{\beta}^{(4)} \partial x_{\beta}^{(4)}} \int \psi^{(4)} F_{4}^{(1,2,3,4)} d v^{(4)} d g^{(4)} d \varphi^{(4)} d \psi^{(4)} \\
-\left[\frac{\partial}{\partial v_{\alpha}^{(1)}}\left\{\frac{1}{4 \pi} \int \frac{\partial}{\partial x_{\alpha}^{(1)}}\left(\frac{1}{\left|\bar{x}^{(4)}-\bar{x}^{(1)}\right|}\right)\right\}+\frac{\partial}{\partial v_{\alpha}^{(2)}}\left\{\frac{1}{4 \pi} \int \frac{\partial}{\partial x_{\alpha}^{(2)}}\left(\frac{1}{\left|\bar{x}^{(4)}-\bar{x}^{(2)}\right|}\right)\right\}+\frac{\partial}{\partial v_{\alpha}^{(3)}}\left\{\frac{1}{4 \pi} \int \frac{\partial}{\partial x_{\alpha}^{(3)}}\left(\frac{1}{\left|\bar{x}^{(4)}-\bar{x}^{(3)}\right|}\right)\right\}\right. \\
\times\left(\frac{\partial v_{\alpha}^{(4)}}{\partial x_{\beta}^{(4)}} \frac{\partial v_{\beta}^{(4)}}{\partial x_{\alpha}^{(4)}}-\frac{\partial g_{\alpha}^{(4)}}{\partial x_{\beta}^{(4)}} \frac{\partial g_{\beta}^{(4)}}{\partial x_{\alpha}^{(4)}}\right) F_{4}^{(1,2,3,4)} \\
\left.\times d x^{(4)} d v^{(4)} d g^{(4)} d \varphi^{(4)} d \psi^{(4)}\right]+\left[f\left(u_{\alpha}^{(1)}-v_{\alpha}^{(1)}\right) \frac{\partial}{\partial v_{\alpha}^{(1)}}+f\left(u_{\alpha}^{(2)}-v_{\alpha}^{(2)}\right) \frac{\partial}{\partial v_{\alpha}^{(2)}}+f\left(u_{\alpha}^{(3)}-v_{\alpha}^{(3)}\right) \frac{\partial}{\partial v_{\alpha}^{(3)}}\right] F_{3}^{(1,2,3)}=0 \\
(61)
\end{gathered}
$$

which was obtained earlier by M. N. Islam et al (2014)

If the fluid is clean then $\mathrm{f}=0$, the transport equation for three-point distribution function in MHD turbulent flow (60) becomes

$$
\frac{\partial F_{3}^{(1,2,3)}}{\partial t}+\left(v_{\beta}^{(1)} \frac{\partial}{\partial x_{\beta}^{(1)}}+v_{\beta}^{(2)} \frac{\partial}{\partial x_{\beta}^{(2)}} v_{\beta}^{(3)} \frac{\partial}{\partial x_{\beta}^{(3)}}\right) F_{3}^{(1,2,3)}+\left[g_{\beta}^{(1)}\left(\frac{\partial g_{\alpha}^{(1)}}{\partial v_{\alpha}^{(1)}}+\frac{\partial v_{\alpha}^{(1)}}{\partial g_{\alpha}^{(1)}}\right) \frac{\partial}{\partial x_{\beta}^{(1)}}\right.
$$




$$
\begin{aligned}
& \left.+g_{\beta}^{(2)}\left(\frac{\partial g_{\alpha}^{(2)}}{\partial v_{\alpha}^{(2)}}+\frac{\partial v_{\alpha}^{(2)}}{\partial g_{\alpha}^{(2)}}\right) \frac{\partial}{\partial x_{\beta}^{(2)}}+g_{\beta}^{(3)}\left(\frac{\partial g_{\alpha}^{(3)}}{\partial v_{\alpha}^{(3)}}+\frac{\partial v_{\alpha}^{(3)}}{\partial g_{\alpha}^{(3)}}\right) \frac{\partial}{\partial x_{\beta}^{(3)}}\right] F_{3}^{(1,2,3)}
\end{aligned}
$$

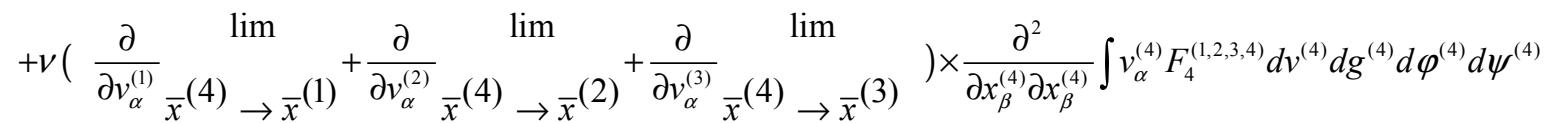

$$
\begin{aligned}
& +\lambda\left(\frac{\partial}{\partial g_{\alpha}^{(1)}} \bar{x}^{(4)} \rightarrow \bar{x}^{(1)}+\frac{\partial}{\partial g_{\alpha}^{(2)}} \bar{x}^{(4)} \lim _{\bar{x}^{(2)}}+\frac{\partial}{\partial g_{\alpha}^{(3)}} \bar{x}^{(4)} \rightarrow \bar{x}^{(3)}\right) \times \frac{\partial^{2}}{\partial x_{\beta}^{(4)} \partial x_{\beta}^{(4)}} \int g_{\alpha}^{(4)} F_{4}^{(1,2,3,4)} d v^{(4)} d g^{(4)} d \varphi^{(4)} d \psi^{(4)} \\
& +\gamma\left(\frac{\partial}{\partial \varphi^{(1)}} \bar{x}^{(4)} \lim _{\bar{x}^{(1)}}+\frac{\partial}{\partial \varphi^{(2)}} \lim _{\bar{x}^{(4)} \rightarrow \bar{x}^{(2)}}+\frac{\partial}{\partial \varphi^{(3)}} \bar{x}^{(4)} \rightarrow \bar{x}^{(3)}\right) \times \frac{\partial^{2}}{\partial x_{\beta}^{(4)} \partial x_{\beta}^{(4)}} \int \varphi^{(4)} F_{4}^{(1,2,3,4)} d v^{(4)} d g^{(4)} d \varphi^{(4)} d \psi^{(4)} \\
& +D\left(\frac{\partial}{\partial \psi^{(1)}} \bar{x}^{(4)} \rightarrow \bar{x}^{(1)}+\frac{\partial}{\partial \psi^{(2)}} \lim _{\bar{x}}^{(4)} \rightarrow \bar{x}^{(2)}+\frac{\partial}{\partial \psi^{(3)}} \bar{x}^{(4)} \rightarrow \bar{x}^{(3)}\right) \times \frac{\partial^{2}}{\partial x_{\beta}^{(4)} \partial x_{\beta}^{(4)}} \int \psi^{(4)} F_{4}^{(1,2,3,4)} d v^{(4)} d g^{(4)} d \varphi^{(4)} d \psi^{(4)} \\
& -\left[\frac{\partial}{\partial v_{\alpha}^{(1)}}\left\{\frac{1}{4 \pi} \int \frac{\partial}{\partial x_{\alpha}^{(1)}}\left(\frac{1}{\left|\bar{x}^{(4)}-\bar{x}^{(1)}\right|}\right)\right\}+\frac{\partial}{\partial v_{\alpha}^{(2)}}\left\{\frac{1}{4 \pi} \int \frac{\partial}{\partial x_{\alpha}^{(2)}}\left(\frac{1}{\left|\bar{x}^{(4)}-\bar{x}^{(2)}\right|}\right)\right\}\right. \\
& \left.+\frac{\partial}{\partial v_{\alpha}^{(3)}}\left\{\frac{1}{4 \pi} \int \frac{\partial}{\partial x_{\alpha}^{(3)}}\left(\frac{1}{\left|\bar{x}^{(4)}-\bar{x}^{(3)}\right|}\right)\right\} \times\left(\frac{\partial v_{\alpha}^{(4)}}{\partial x_{\beta}^{(4)}} \frac{\partial v_{\beta}^{(4)}}{\partial x_{\alpha}^{(4)}}-\frac{\partial g_{\alpha}^{(4)}}{\partial x_{\beta}^{(4)}} \frac{\partial g_{\beta}^{(4)}}{\partial x_{\alpha}^{(4)}}\right) F_{4}^{(1,2,3,4)} \times d x^{(4)} d v^{(4)} d g^{(4)} d \varphi^{(4)} d \psi^{(4)} \quad\right]
\end{aligned}
$$

It was obtained earlier by Azad et al (2014a)

If we drop the viscous, magnetic and thermal diffusive and concentration terms from the three point evolution equation (60), we have

$$
\begin{gathered}
\frac{\partial F_{3}^{(1,2,3)}}{\partial t}+\left(v_{\beta}^{(1)} \frac{\partial}{\partial x_{\beta}^{(1)}}+v_{\beta}^{(2)} \frac{\partial}{\partial x_{\beta}^{(2)}} v_{\beta}^{(3)} \frac{\partial}{\partial x_{\beta}^{(3)}}\right) F_{3}^{(1,2,3)}+\left[g_{\beta}^{(1)}\left(\frac{\partial g_{\alpha}^{(1)}}{\partial v_{\alpha}^{(1)}}+\frac{\partial v_{\alpha}^{(1)}}{\partial g_{\alpha}^{(1)}}\right) \frac{\partial}{\partial x_{\beta}^{(1)}}\right. \\
\left.+g_{\beta}^{(2)}\left(\frac{\partial g_{\alpha}^{(2)}}{\partial v_{\alpha}^{(2)}}+\frac{\partial v_{\alpha}^{(2)}}{\partial g_{\alpha}^{(2)}}\right) \frac{\partial}{\partial x_{\beta}^{(2)}}+g_{\beta}^{(3)}\left(\frac{\partial g_{\alpha}^{(3)}}{\partial v_{\alpha}^{(3)}}+\frac{\partial v_{\alpha}^{(3)}}{\partial g_{\alpha}^{(3)}}\right) \frac{\partial}{\partial x_{\beta}^{(3)}}\right] F_{3}^{(1,2,3)} \\
-\left[\frac{\partial}{\partial v_{\alpha}^{(1)}}\left\{\frac{1}{4 \pi} \int \frac{\partial}{\partial x_{\alpha}^{(1)}}\left(\frac{1}{\left|\bar{x}^{(4)}-\bar{x}^{(1)}\right|}\right)\right\}+\frac{\partial}{\partial v_{\alpha}^{(2)}}\left\{\frac{1}{4 \pi} \int \frac{\partial}{\partial x_{\alpha}^{(2)}}\left(\frac{1}{\left|\bar{x}^{(4)}-\bar{x}^{(2)}\right|}\right)\right\}\right. \\
\left.+\frac{\partial}{\partial v_{\alpha}^{(3)}}\left\{\frac{1}{4 \pi} \int \frac{\partial}{\partial x_{\alpha}^{(3)}}\left(\frac{1}{\left|\bar{x}^{(4)}-\bar{x}^{(3)}\right|}\right)\right\} \times\left(\frac{\partial v_{\alpha}^{(4)}}{\partial x_{\beta}^{(4)}} \frac{\partial v_{\beta}^{(4)}}{\partial x_{\alpha}^{(4)}}-\frac{\partial g_{\alpha}^{(4)}}{\partial x_{\beta}^{(4)}} \frac{\partial g_{\beta}^{(4)}}{\partial x_{\alpha}^{(4)}}\right) F_{4}^{(1,2,3,4)} \times d x^{(4)} d v^{(4)} d g^{(4)} d \varphi^{(4)} d \psi^{(4)} \quad\right]=0
\end{gathered}
$$

The existence of the terms

$$
\left(\frac{\partial g_{\alpha}^{(1)}}{\partial v_{\alpha}^{(1)}}+\frac{\partial v_{\alpha}^{(1)}}{\partial g_{\alpha}^{(1)}}\right),\left(\frac{\partial g_{\alpha}^{(2)}}{\partial v_{\alpha}^{(2)}}+\frac{\partial v_{\alpha}^{(2)}}{\partial g_{\alpha}^{(2)}}\right) \text { and }\left(\frac{\partial g_{\alpha}^{(3)}}{\partial v_{\alpha}^{(3)}}+\frac{\partial v_{\alpha}^{(3)}}{\partial g_{\alpha}^{(3)}}\right)
$$

can be explained on the basis that two characteristics of the flow field are related to each other and describe the interaction between the two modes (velocity and magnetic) at point $\mathrm{x}^{(1)}, \mathrm{x}^{(2)}$ and $\mathrm{x}^{(3)}$.

We can exhibit an analogy of this equation with the $1^{\text {st }}$ equation in BBGKY hierarchy in the kinetic theory of gases. The first equation of BBGKY hierarchy is given by Lundgren (1969) as

$$
\frac{\partial F_{1}^{(1)}}{\partial t}+\frac{1}{m} v_{\beta}^{(1)} \frac{\partial}{\partial x_{\beta}^{(1)}} F_{1}^{(1)}=n \iint \frac{\partial \psi_{1,2}}{\partial x_{\alpha}^{(1)}} \frac{\partial F_{2}^{(1,2)}}{\partial v_{\alpha}^{(1)}} d \bar{x}^{(2)} d \bar{v}^{(2)}
$$

where $\psi_{1,2}=\psi\left|v_{\alpha}^{(2)}-v_{\alpha}^{(1)}\right|$ is the inter molecular potential.

\section{Acknowledgement}

Authors are grateful to the Department of Applied Mathematics, University of Rajshahi, Bangladesh for giving all facilities and support to carry out this work.

\section{References}

[1] Hopf E. "Statistical hydrodynamics and functional calculus", J. Rotational Mech. Anal., 1: 87-123, 1952.

[2] Kraichanan R H., "Distribution functions in the statistical theory of convective MHD turbulent flow", J. Fluid Mech., 5, 497, 1959.

[3] Edward S., "The statistical dynamics of homogeneous turbulence" J. Fluid Mech. 18(2): 239-273, 1964. 
[4] Herring J. R. "Self-consistent field approach to turbulence theory", Phys. Fluid, 8: 2219-2225, 1965.

[5] Ta-You W., "Kinetic Theory of Gases and Plasma". Addision Wesley Phlelishing, 1966.

[6] Lundgren T.S., Hierarchy of coupled equations for multi-point turbulence velocity distribution functions. Phys. Fluid., 10: 967. 1967.

[7] Lundgren T.S., "Hierarchy of coupled equations for multipoint turbulence velocity distribution functions". Phys. Fluid., 12: 485.1969.

[8] Bigler R. W., "The structure of diffusion flames. Combustion Sci. and Tech.13: 155. 1976

[9] Kishore N., "Distribution functions in the statistical theory of MHD turbulence of an incompressible fluid". J. Sci. Res., BHU, 28(2): 163. 1978.

[10] Pope S. B., "The statistical theory of turbulence flames". Phil. Trans Roy. Soc. London A, 291: 529. 1979.

[11] Pope S.B., "The transport equation for the joint probability density function of velocity and scalars in turbulent flow". Phys. Fluid., 24: 588. 1981.

[12] Kollman W. and J. Janica, "The transport equation for the probability density function of a scalar in turbulent shear flow”. Phys. Fluid., 25: 1955. 1982.

[13] Kishore N, and S. R. Singh. "Transport equation for the bivariate joint distribution function of velocity and temperature in turbulent flow”. Bull. Tech. Univ. Istambul, 37: 91-100. 1984.

[14] Kishore N., and S. R. Singh, "Transport equation for the joint distribution function of velocity, temperature and concentration in convective turbulent flow. Prog. of Maths. 19(1\&2):13-22. 1985.

[15] Dixit T. \& B.N. Upadhyay, 1989 "Distribution functions in the statistical theory of MHD turbulence of an incompressible fluid in the presence of the Coriolis force", Astrophysics and Space Sci., 153: 297-309.

[16] Sarker M.S.A. and N. Kishore, "Distribution functions in the statistical theory of convective MHD turbulence of an incompressible fluid". Astrophys \& Space Sci., 181: 29. 1991.

[17] Sarker M.S.A. and N. Kishore, "Distribution functions in the statistical theory of convective MHD turbulence of mixture of a miscible incompressible fluid". Prog. Math., BHU India, 33(1-2): 83. 1999.

[18] Sarker M.S.A. and M.A. Islam, "Distribution functions in the statistical theory of convective MHD turbulence of an incompressible fluid in a rotating system". Rajshahi University Studies. Part-B. J. Sci., 30: 2002.

[19] Azad, M. A. K. \& M. S. A. Sarker, "Decay of MHD turbulence before the final period for the case of multi-point and multi-time in presence of dust particle". Bangladesh J. Sci. Ind. Res. 38(3-4):151-164. 2003.

[20] Azad, M. A. K. \& M. S. A. Sarker, "Statistical theory of certain distribution functions in MHD turbulence in a rotating system in presence of dust particles". Rajshahi university studies". Part -B. J. Sci., 32: 193-210. 2004a.
[21] Sarker M. S. A. \& M. A. K Azad, "Decay of MHD turbulence before the final period for the case of multi-point and multitime in a rotating system". Rajshahi. University Studies. PartB, J. Sci., 32: 177-192. 2004b.

[22] Sarker M. S. A. \& M. A. K. Azad, "Decay of temperature fluctuations in homogeneous turbulence before the final period for the case of multi-point and multi-time in a rotating system". Bangladesh. J. Sci. \& Ind. Res., 41 (3-4): 147-158. 2006.

[23] Islam M.A. and M. S. A. Sarker, "Distribution functions in the statistical theory of MHD turbulence for velocity and concentration undergoing a first order reaction". Rajshahi university studies. Part-B. J. Sci., 35: 2007.

[24] Azad, M. A. K. \& M. S. A. Sarker, "Decay of temperature fluctuations in homogeneous turbulence before the final period for the case of Multi- point and Multi- time in a rotating system in presence of dust particle". J. Appl. Sci. Res., 4(7): 793- 802. 2008.

[25] Azad, M. A. K. \& M. S. A. Sarker, "Decay of temperature fluctuations in MHD turbulence before the final period in a rotating system". Bangladesh J. Sci \& Ind. Res, 44(4): 407414. 2009a.

[26] Azad M. A. K., Aziz M. A., \& M. S. A. Sarker, "First order reactant in Magneto-hydrodynamic turbulence before the final Period of decay in presence of dust particles in a rotating System”. J. Phy. Sci., 13: 175-190. 2009b.

[27] Azad M. A. K., M. A. Aziz \& M. S. A. Sarker, "First order reactant in MHD turbulence before the final period of decay in a rotating system”. J. Mech. Contin. \& Math. Sci., 4(1): 410417. 2009c.

[28] Aziz M. A., M. A. K. Azad, \& M. S. A. Sarker, "First order reactant in Magneto- hydrodynamic turbulence before the final period of decay for the case of multi-point and multi-time in a rotating system”. Res. J. Math. \& Stat., 1(2): 35-46. 2009c.

[29] Aziz M. A., M. A. K Azad, \& M. S. A. Sarker, "Statistical Theory of Certain Distribution Functions in MHD Turbulent Flow Undergoing a First Order Reaction in Presence of Dust Particles", Journal of Modern Mathematics and Statistics, 4(1):11-21. 2010a.

[30] Aziz M. A., M. A. K Azad, \& M. S. A. Sarker, "Statistical Theory of Distribution Functions in Magneto-hydrodynamic Turbulence in a Rotating System Undergoing a First Order Reaction in Presence of Dust Particles". Res. J. Mathematics and Statistics, 2(2):37-55. $2010 \mathrm{~b}$.

[31] Aziz M. A., M. A. K Azad, \& M. S. A. Sarker,. "First order reactant in MHD turbulence before the final period of decay for the case of multi-point and multi-time in a rotating system in presence of dust particle". Res. J. Math. \& Stat., 2(2): 56$68,2010 \mathrm{c}$.

[32] Azad, M. A. K., M. A. Aziz \& M. S. A. Sarker, "Statistical Theory of certain Distribution Functions in MHD Turbulent flow for Velocity and Concentration Undergoing a First Order Reaction in a Rotating System". Bangladesh J. Scientific \& Ind. Res., 46(1): 59-68. 2011.

[33] Azad, M. A. K., M. H. U. Molla \& M. Z. Rahman, "Transport Equatoin for the Joint Distribution Function of Velocity, Temperature and Concentration in Convective Tubulent Flow in Presence of Dust Particles”. Res.J. Appl.Sci., Engng. Tech. 4(20): 4150-4159. 2012. 
[34] Molla M. H. U., M. A. K Azad, \& M. Z. Rahman, ”Decay of temperature fluctuations in homogeneous turbulenc before the finaln period in a rotating system". Res. J. Math. \& Stat., 4(2): 45-51. 2012 .

[35] Bkar Pk M. A., M. A. K Azad \& M. S. A. Sarker, "First-order reactant in homogeneou dusty fluid turbulence prior to the ultimate phase of decay for four-point correlation in a rotating system. Res. J. Math. \& Stat., 4(2): 30-38. 2012.

[36] Bkar Pk M. A., M. A. K Azad, \& M. S. A. Sarker, "First-order reactant in homogeneous turbulence prior to the ultimate phase of decay for four-point correlation in presence of dust particle”. Res. J. Appl. Sci. Engng. \& Tech., 5(2): 585-595. 2013a.

[37] Bkar Pk. M. A., M. S. A. Sarker \& M. A. K. Azad, "Homogeneous turbulence in a first-order reactant for the case of multi-point and multi-time prior to the final period of decay in a rotating system". Res. J. Appl. Sci., Engng. \& Tech. 6(10): 1749-1756. 2013b.

[38] Bkar Pk. M. A., M. S. A. Sarker \& M. A. K. Azad, "Decay of MHD turbulence before the final period for four- point correlation in a rotating system”. Res. J. Appl. Sci., Engng. \& Tech. 6(15): 2789-2798. 2013c.

[39] Bkar Pk M. A., M. A. K Azad, \& M. S. A. Sarker, "Decay of dusty fluid MHD turbulence for four- point correlation in a rotating System”. J. Sci. Res. 5 (1):77-90. 2013d.

[40] Azad, M. A. K., M. H. U. Molla \& M. Z. Rahman, "Transport Equatoin for the Joint Distribution Functions in Convective Tubulent Flow in Presence of Dust Particles undergoing a first order reaction”. Rajshahi Univ. J. Sci. \& Engng( accepted for publication), volume 41: 2014.

[41] Azad M. A. K. \& Mst. Mumtahinah, "Decay of Temperature fluctuations in dusty fluid homogeneous turbulence prior to final period, Res. J. Appl. Sci., Engng and Tech., 6(8): 14901496. 2013.

[42] Bkar PK. M. A., M. S. A. Sarker \& M. A. K Azad, "Decay of MHD Turbulence prior to the ultimate phase in presence of dust particle for Four- point Correlation”. Int. J. of Appl. Math and Mech. 9 (10): 34-57. 2013.
[43] Molla M. H. U., M. A. K. Azad, \& M. Z. Rahman, "Transport equation for the joint distribution functions of velocity, temperature and concentration in convective turbulent flow in presence of coriolis force". Int. J. Scholarly Res. Gate, 1(4): 42-56. 2013.

[44] Azad M. A. K. \& Mst. Mumtahinah, "Decay of Temperature fluctuations in dusty fluid homogeneous turbulence prior to the ultimate period in presence of coriolis force". Res. J. Appl. Sci. Engng. and Tech. 7(10):1932-1939. 2014.

[45] Azad M. A. K., M. N. Islam \& Mst. Mumtahinah, "Transport Equations of Three- point Distribution Functions in MHD Turbulent Flow for Velocity, Magnetic Temperature and Concentration". Res. J. Appl. Sci. Engng. and Tech. 7(24): 5184-5220. 2014a.

[46] Azad M. A. K. \& M. N. Islam, "Transport Equations of Three Point Distribution Functions in MHD Turbulent Flow for Velocity, Magnetic Temperature and Concentration in a Rotating System". Int. J. Scholarly Res. Gate, 2(3): 75-120. 2014b.

[47] Islam M. N. \& M. A. K Azad, "Transport equations of threepoint distribution functions in MHD turbulent flow for velocity, magnetic temperature and concentration in presence of dust particles", Int. J. Scholarly Res. Gate, 2(4): 195-240. 2014.

[48] Bkar Pk M. A., A. Malek \& M. A. K. Azad, "Effects of firstorder reactant on MHD turbulence at four-point correlation". Applied and Computational Mathematics, 4(1): 11-19, 2015.

[49] M. A. K. Azad, Mst. Mumtahinah, \& M. A. Bkar Pk, "Transport equation for the joint distribution functions of certain variables in convective dusty fluid turbulent flow in a rotating system undergoing a first order reaction". American Journal of Applied Mathematics, 3(1): 21-30, 2015.

[50] Bkar Pk M. A., A. Malek and M. A. K. Azad, "4-point correlations of dusty fluid MHD turbulent flow in a $1^{\text {st }}$ order reaction". Global J. of Science Frontier Research :F (Mathematics and Decision Sciences ) 15(2), version 1.0: 53$69,2015(\mathrm{a})$ 